\title{
Approximate quantum and acoustic cloaking
}

\author{
Allan Greenleaf, Yaroslav Kurylev, \\ Matti Lassas and Gunther Uhlmann ${ }^{1}$
}

\begin{abstract}
For any $E \geq 0$, we construct a sequence of bounded potentials $V_{n}^{E}, n \in \mathbb{N}$, supported in an annular region $B_{\text {out }} \backslash B_{\text {in }} \subset \mathbb{R}^{3}$, which act as approximate cloaks for solutions of Schrödinger's equation at energy $E$ : for any potential $V_{0} \in L^{\infty}\left(B_{\text {in }}\right)$ such that $E$ is not a Neumann eigenvalue of $-\Delta+V_{0}$ in $B_{\text {in }}$, the scattering amplitudes $a_{V_{0}+V_{n}^{E}}(E, \theta, \omega) \rightarrow 0$ as $n \rightarrow \infty$. The $V_{n}^{E}$ thus not only form a family of approximately transparent potentials, but also function as approximate invisibility cloaks in quantum mechanics. On the other hand, for $E$ close to interior eigenvalues, resonances develop and there exist almost trapped states concentrated in $B_{\text {in }}$. We derive the $V_{n}^{E}$ from singular, anisotropic transformation optics-based cloaks by a de-anisotropization procedure, which we call isotropic transformation optics. This technique uses truncation, inverse homogenization and spectral theory to produce nonsingular, isotropic approximate cloaks. As an intermediate step, we also obtain approximate cloaking for a general class of equations including the acoustic equation.
\end{abstract}

Mathematics Subject Classification (2010). Primary 35R30; Secondary 35Q60, 35Q40.

Keywords. Invisibility cloaking, quantum mechanics, Helmholtz equation, homogenization.

\section{Contents}

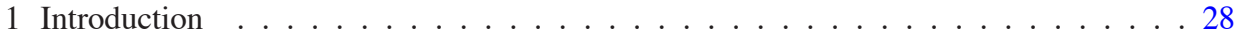

2 Approximating cloaking material parameters by nonsingular anisotropic parameters . 31

3 Approximating anisotropic by isotropic mass densities . . . . . . . . . . . . . . 49

4 Approximate acoustic cloaking . . . . . . . . . . . . . 661

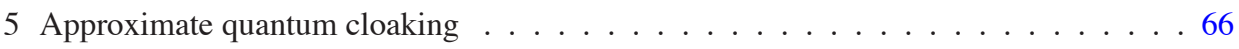

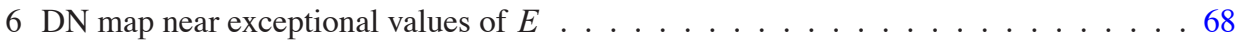

7 Numerical results . . . . . . . . . . . . . . . . . . . . . . 73

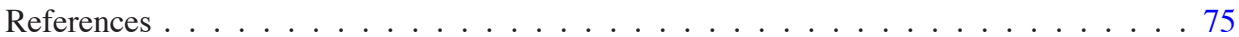

\footnotetext{
${ }^{1}$ A. Greenleaf and G. Uhlmann were supported by the US NSF, Y. Kurylev by EPSRC, M. Lassas by Academy of Finland (CoE project 213476), and G. Uhlmann by a Walker Family Professorship. The authors thank A. Cherkaev and V. Smyshlyaev for useful discussions on homogenization.
} 


\section{Introduction}

A fundamental problem is to describe the scattering of waves by a potential, as governed by the time-independent Schrödinger equation at energy $E \geq 0$,

$$
(-\Delta+V(x)) \psi(x)=E \psi(x), \quad x \in \mathbb{R}^{d},
$$

where $x \in \mathbb{R}^{d}, \psi(x)=\exp \left(i E^{1 / 2} x \cdot \theta\right)+\psi_{\mathrm{sc}}(x), \theta \in \mathbb{S}^{d-1}$, and $\psi_{\mathrm{sc}}(x)$ satisfies the Sommerfeld radiation condition. We restrict ourselves in this paper to compactly supported potentials $V$, so

$$
\psi_{\mathrm{sc}}(x)=C_{d} E^{\frac{d-3}{4}} \frac{a_{V}\left(E, \frac{x}{|x|}, \theta\right)}{|x|^{\frac{d-1}{2}}} \exp \left(i E^{1 / 2}|x|\right)+o\left(|x|^{-\frac{d-1}{2}}\right), \quad \text { as }|x| \rightarrow \infty .
$$

The function $a_{V}(E, \omega, \theta)$ is the scattering amplitude at energy $E$ of the potential $V$. The associated inverse scattering problem consists of trying to determine $V$ from the scattering amplitude, or measurements of waves at the boundary of some region $\Omega$ containing the support of $V$.

Recently, [22] described quantum mechanical cloaking at any fixed energy $E$. Their construction starts with a homogeneous, isotropic mass tensor and a potential $V \equiv 0$, and subjects this pair to a singular change of variables. This "blowing up a point" transformation had been used in [34] and [35] to produce conductivities that hide objects from detection by electrostatic measurements, ${ }^{2}$ and was subsequently used to describe the same phenomenon for electromagnetic waves [62]; one now refers to a specification of material parameters having this effect as a cloak. The cloaking Schrödinger equation in [22], which has an anisotropic, singular mass tensor, is equivalent with the Helmholtz equation (at frequency $\omega=\sqrt{E}$ ) for an associated singular Riemannian metric, and thus covered by cloaking for the Helmholtz equation in 3D, as we analyzed in Section 3 in [24]. Similarly, cloaks for acoustics in 3D have been described in [11] and [17]; ${ }^{3}$ again, these are in fact direct consequences of cloaking for the Helmholtz equation in 3D, cf. [28].

Thus, for ideal 3D cloaking in each of scalar optics, quantum mechanics and acoustics, one knows from [24] that any finite energy distributional solution decouples into a sum of a wave on the exterior of the cloak, unaffected (in terms of scattering or boundary measurements) by the cloak, and a wave within the cloaked region satisfying the Neumann boundary condition at the cloaking surface. Hence, if $E$ is not a Neumann eigenvalue, then the wave must vanish within the cloaked region and cloaking works as advertised. On the other hand, if $E$ is an eigenvalue, the cloaked region supports interior resonances, or trapped states. This is an unphysical situation, since the Dirichlet problem on $\Omega$ no longer has unique solutions, and this can be considered as a failure of cloaking, per se. However, what emerges from this

\footnotetext{
${ }^{2}$ The $2 \mathrm{D}$ version has the same electrostatic cloaking property [42].

${ }^{3}$ See [16] for the 2D case, and [52] and [58] for more regarding elastic and acoustic cloaking.
} 
failure in the setting of approximate cloaking described below is, we believe, quite useful.

In this paper, we construct for the Schrödinger equation, for each energy $E$, a family $\left\{V_{n}^{E}\right\}_{n=1}^{\infty}$ of potentials, supported in an annulus $B_{\text {out }} \backslash B_{\text {in }} \subset \mathbb{R}^{3}$, which are not only almost transparent, in that the scattering amplitudes $a_{V_{n}^{E}}(E, \cdot, \cdot) \rightarrow 0$ as $n \rightarrow \infty$, but also act as approximate cloaks for potentials supported in the inner ball $B_{\text {in }}$ : for any potential $V_{0} \in L^{\infty}\left(B_{\text {in }}\right)$ for which $E$ is not an eigenvalue of $-\Delta+V_{0}$ in $B_{\text {in }}$, the scattering amplitudes $a_{V_{0}+V_{n}^{E}}(E, \cdot, \cdot) \rightarrow 0$ as $n \rightarrow \infty$, as well. There are also approximate versions of the interior resonances supported by the ideal cloak: there exist energies close to the Neumann eigenvalues of the cloaked region $B_{\text {in }}$, near which there are waves largely concentrated in $B_{\text {in }}$, which we call almost trapped states. Furthermore, the quality of the approximate cloaking degrades as we move towards these energies, with waves being able to penetrate $B_{\text {in }}$, and the presence of this region and $V_{0}$ detectable by scattering or boundary measurements. In addition, we are able to include a magnetic potential in the Schrödinger equation; this allows one to switch between the approximate cloak and almost trapped state alternatives by application of a suitable homogeneous magnetic field. We have given physical applications of this in [29] and [30].

The inverse problem of recovering a potential from the Dirichlet-to-Neumann map (near field) or scattering amplitude (far field) associated to the Schrödinger equation has been extensively studied in the mathematical literature, see, e.g., [7], [70], [59], [46], [55], [9], [20], [54], [69], and [8]. Unique identifiability of the potential, as well as counterexamples, have been shown under various regularity assumptions in [65], [56], [67], [36], [60], [33], and [37].

We construct the families $\left\{V_{n}^{E}\right\}$ of approximately cloaking potentials by means of a result of independent interest. The use of changes of variables to produce novel optical effects on waves or to facilitate computations has been considered in the physics literature (see, e.g., Dolin [19] ${ }^{4}$ or more recently Ward and Pendry [72]) and is now generally referred to as transformation optics (TO). However, to produce cloaking and other extreme effects, nonsingular changes of variables are insufficient. The recently proposed plans for cloaking are based on singular transformations and consist of medium parameters which are both anisotropic and singular, ${ }^{5}$ whether for the conductivity (electrostatics) [34] and [35], index of refraction (Helmholtz) [48], [24], and [41], permittivity and permeability (Maxwell) [62] and [24], mass density (acoustic) [16], [11], [17], and [28], or effective mass (Schrödinger) [22]. Physical realization of such designs is now potentially feasible due to the rapidly developing area of metamaterials, but the singularity and extreme anisotropy make characterizing and fabricating the materials to implement such designs an enormous obstacle to manufacturing invisibility devices. These same remarks are valid for other TO designs, such as [49], [10], [14], [25], [27], and [63], some of which are singular.

\footnotetext{
${ }^{4}$ We thank A. Kildishev for this reference.

${ }^{5} \mathrm{By}$ singular we mean that at least one of the eigenvalues goes to zero or infinity at some points.
} 
We propose here a general method, which we refer to by the oxymoronic isotropic transformation optics, for dealing with both the anisotropy and singularity of TO material parameters. We describe this in detail in the context of cloaking, but it should be applicable to a wider range of TO designs. We in fact derive the quantum mechanical approximate cloaks from approximate cloaks for a general class of equations that includes the acoustic equation. Using ideas from Nguetseng [57], Allaire [1], Cherkaev [15], and elsewhere, we show how to find cloaking material parameters that are at once both isotropic and nonsingular, at the price of replacing perfect (ideal) cloaking with approximate cloaking (of arbitrary accuracy). This method, starting with transformation optics-based designs and constructing approximations to them, first by nonsingular, but still anisotropic, material parameters, and then by nonsingular isotropic parameters, appears to be a very flexible tool for creating physically realistic theoretical designs, easier to implement than the ideal ones due to the relatively tame nature of the materials needed, yet (up to an arbitrarily small error) essentially capturing the desired effects on wave propagation.

We review the ideal electrostatic cloak of [34], and extend this in Section 2.1 to a class of equations which will allow us to deal with both general acoustic and magnetic Schrödinger equations. These equations have coefficients which, borrowing the terminology from acoustics, we refer to as mass density and bulk modulus and which are singular at the cloaking surface $\Sigma$, i.e. the interface between the cloaked and uncloaked regions. The desingularization process begins in Section 2.2, by truncating the mass density away from $\Sigma$, on the outer side of which the ideal cloaking parameters are singular. (Similar truncations have been considered before in the context of cylindrical or 2D cloaking, cf. [66], [26], [12], and [42].) We show in Section 2.3 that the Dirichlet forms for the ideal cloaks are well approximated (in the sense of $\Gamma$-convergence) by these truncations, and then desingularize the bulk modulus in Section 2.4.

So far, the approximately cloaking mass densities are still anisotropic. As is well known in effective medium theory, homogenization of isotropic material parameters may lead to anisotropic ones [51]; in Section 3 and Section 4, we use this phenomenon in reverse, showing that the Dirichlet forms obtained in Section 2 can be well approximated by those for certain nonsingular isotropic conductivities, which thus provide approximate cloaks for the general class of acoustic-like equations. These then allow us to obtain in Theorem 5.1 families of approximate quantum cloaks. In Section 6 we study failure of cloaking near exceptional energies, mirroring the failure of the existence of unique solutions for the ideal cloak at Neumann eigenvalues of the cloaked region; see Remark 6.2 at the end of the section. We point out that these almost trapped states make sense for waves modeled by the Helmholtz equation, such as in acoustics, but for simplicity we present the details only for the Schrödinger equation. Further physical applications, including a new type of ion trap, can be found in [29] and [30]. Finally, numerical simulations are presented in Section 7.

One can use the resonances of the approximative cloaks analyzed in this paper to construct various physical applications, for instance, to create almost invisible sen- 
sors, that is sensors which cause only an arbitrarily small perturbation for the measured field. Such sensors based on materials with negative refractive index have been proposed earlier [3], but using the approximate cloaks, the sensors can be obtained using materials with strictly positive refractive index. Indeed, an almost invisible sensor can be obtained by placing a carefully chosen object inside an approximative cloak so that the interior of the cloak is not in a resonance but very close to it. Then, the object is almost cloaked but the external field penetrates inside the cloaked region, see [31]. Such a construction is an example how delicate phenomena may happen for an invisibility cloak near a resonance.

\section{Approximating cloaking material parameters by nonsingular anisotropic parameters}

We will denote by $B(a, R)$ the ball of radius $R$ centered at $a$ in $\mathbb{R}^{3}$, sometimes denoted simply $B(R)$ when centered at the origin $O$. Let $M_{1}=\bar{B}\left(O_{1}, 3\right) \subset \mathbb{R}^{3}$ and $M_{2}=\bar{B}\left(O_{2}, 1\right)$, with $O_{1}$ and $O_{2}$ being two copies of $O$, considered as disjoint compact manifolds with boundary; set $M=M_{1} \cup M_{2}$. Also, let $\Omega=\bar{B}(3) \subset \mathbb{R}^{3}$, and $F^{1}: M_{1} \backslash\left\{O_{1}\right\} \rightarrow \Omega \backslash \bar{B}(1) \subset \mathbb{R}^{3}$ be the map

$$
\begin{array}{ll}
F^{1}(x)=x & \text { for } 2<|x| \leq 3, \\
F^{1}(x)=\left(1+\frac{|x|}{2}\right) \frac{x}{|x|} & \text { for } 0<|x| \leq 2 .
\end{array}
$$

Define also $F^{2}: M_{2} \rightarrow \bar{B}(1)$ as the identity map,

$$
F^{2}(x)=x .
$$

Together, these form a surjective map $F=\left(F^{1}, F^{2}\right)$ from the cloaking manifold (or virtual space) $M \backslash\left\{O_{l}\right\}$ to the cloaking device (or physical space) $\Omega$. By a conductivity we mean a measurable map with values in the symmetric non-negative $\mathbb{R}^{3 \times 3}$ matrices. Let $\gamma_{0}=1$ be the constant isotropic conductivity on $\mathbb{R}^{3}$ and define the conductivity $\sigma_{1}$ on $\Omega$ as

$$
\begin{array}{ll}
\sigma_{1}=F_{*}^{1} \gamma_{0} & \text { for } x \in \Omega \backslash \bar{B}(1), \\
\sigma_{1}=2 F_{*}^{2} \gamma_{0} & \text { for } x \in B(1),
\end{array}
$$

which has a singularity on the cloaking surface $\Sigma=\partial B(1)$, both in that one of the eigenvalues (corresponding to the radial direction) tends to 0 as $r \searrow 1$ and that there is a jump discontinuity across the sphere $\Sigma$. This conductivity $\sigma_{1}$ is, up to the radius of $\Omega$ and the factor 2 in the second of formulae (4), used here for technical reasons, the one introduced in [34] and [35] and shown to be indistinguishable from $\gamma_{0}$, vis-a-vis electrostatic boundary measurements at $\partial \Omega$. In fact, $\left.\sigma_{1}\right|_{B(1)}$ can be replaced by any smooth, non-degenerate anisotropic conductivity tensor and its values will be 
undetectable at $\partial \Omega$. The same construction of $\left.\sigma_{1}\right|_{\Omega \backslash B(1)}$, applied instead to the electric permittivity $\varepsilon$ and magnetic permeability $\mu$ in Maxwell's equations, was proposed in [62] (see also [48]) to cloak the region $B(1)$ from observation by electromagnetic waves at positive frequency; we thus refer to $\sigma_{1}$ as a cloaking conductivity, and, following the physics literature, we will refer to $\left(\Omega, \sigma_{1}\right)$ as the ideal cloak.

This gives rise to the Dirichlet problem for the singular conductivity equation,

$$
\nabla \cdot \sigma_{1} \nabla u=0 \text { in } \Omega,\left.\quad u\right|_{\partial \Omega}=h,
$$

and to the corresponding singular conductivity operator $\mathcal{A}$,

$$
\mathcal{A} u=-g^{-1 / 2} \nabla \cdot \sigma_{1} \nabla u,
$$

that we consider with Dirichlet boundary condition $\left.u\right|_{\partial \Omega}=0$. Here, we use the singular Riemannian metric $\left(g_{j k}\right)_{j, k=1}^{3}$ associated to the conductivity $\sigma_{1}$, namely,

$$
g^{1 / 2} g^{j k}=\sigma_{1}^{j k}, \quad g=\left|\operatorname{det}\left[g^{i j}\right]\right|^{-1}=\left|\operatorname{det}\left[\sigma_{1}^{i j}\right]\right|^{2},
$$

cf. [34] and [35]. We denote by $g$ both the metric and the corresponding scalar function, the meaning being clear from the context.

A rigorous definition of the meaning of (5) and the operator $\mathcal{A}$ is given in the following sections. In particular, the operator $\mathcal{A}$ is self-adjoint on $L_{g}^{2}(\Omega)$, the weighted $L^{2}$-space defined using the weight $g^{1 / 2}$. For a general weight $w(x) \geq 0$, we denote by $L^{2}(\Omega, w d x)=L^{2}(w d x)$ the weighted space,

$$
L^{2}(\Omega, w d x)=\left\{u: \Omega \rightarrow \mathbb{C} \text { measurable, }\|u\|_{L^{2}(w d x)}^{2}=\int_{\Omega}|u|^{2} w d x<\infty\right\} .
$$

For simplicity, we denote $L_{g}^{2}=L_{g}^{2}(\Omega)=L^{2}\left(\Omega, g^{1 / 2} d x\right)$, the natural $L^{2}$-space for the metric $g$, and the norm in this space by $\|\cdot\|_{g}$. Note that

$$
L^{2}(\Omega) \subset L_{g}^{2}(\Omega), \quad\|u\|_{g} \leq \sqrt{8}\|u\| .
$$

We also use the Sobolev spaces

$$
\begin{aligned}
H_{g}^{1}(\Omega) & =\left\{u \in L_{g}^{2}(\Omega):\left.u\right|_{\Omega \backslash \Sigma} \in H_{\mathrm{loc}}^{1}(\Omega \backslash \Sigma), \int_{\Omega \backslash \Sigma} \sigma_{1}^{j k} \partial_{j} u \overline{\partial_{k} u} d x<\infty\right\}, \\
H_{0, g}^{1}(\Omega) & =\left\{v \in H_{g}^{1}(\Omega):\left.v\right|_{\partial \Omega}=0\right\} .
\end{aligned}
$$

Here and below, we use Einstein summation convention, summing over indices $j$ and $k$ appearing both as sub- and super-indices. Observe that $H^{1}(\Omega) \subset H_{g}^{1}(\Omega)$ and

$$
\|u\|_{H_{g}^{1}}=\left(\|u\|_{L_{g}^{2}}^{2}+\int_{\Omega} \sigma_{1}^{j k} \partial_{j} u \overline{\partial_{k} u} d x\right)^{\frac{1}{2}} \leq \sqrt{8}\|u\|_{H^{1}} .
$$

Throughout, we also use the following standard terminology: when considering convergence of sequences $\left\{x_{n}\right\}_{n=1}^{\infty}$ in a Hilbert space $H$, we say that $x_{n}$ converges strongly to $x$ in $H$ if $\left\|x_{n}-x\right\|_{H} \rightarrow 0$, as $n \rightarrow \infty$, while $x_{n}$ converges weekly to $x$ if $\left(x_{n}-x, y\right)_{H} \rightarrow 0$, as $n \rightarrow \infty$, for any $y \in H$. 
2.1. Ideal cloaking for more general equations. In this paper we treat equations more general than (5), which are important for physical applications, cf. [29] and [30]. To this end, consider a family of equations that simultaneously includes both the magnetic Schrödinger equation and the acoustic equation; for simplicity, we will refer to equations of this type as acoustic. Let $q$ and $b=\left(b_{1}, b_{2}, b_{3}\right)$ be, respectively, scalar-valued and vector-valued functions, which eventually will represent the electric and magnetic potentials; we will assume that

$$
q \in L^{\infty}(\Omega ; \mathbb{R}), \quad q \geq 0, \quad \text { and } \quad b \in L^{\infty}\left(\Omega ; \mathbb{R}^{3}\right) .
$$

We note that the condition $q \geq 0$ is merely a convenience, since for general $q \in$ $L^{\infty}(\Omega ; \mathbb{R})$ we can always add a constant to achieve positivity, and of course this just shifts the spectrum.

To deal rigorously with the elliptic boundary value problem

$$
-g^{-1 / 2}(\nabla+i b) \cdot \sigma_{1}(\nabla+i b) u+q u-\lambda u=f,\left.\quad u\right|_{\partial \Omega}=h,
$$

where $i b$ acts as a vector-valued multiplication operator. Since (10) has singular coefficients, we consider the corresponding quadratic form. In the following, we use the notation

$$
\nabla^{b}=\nabla+i b(x)
$$

Lemma 2.1. The quadratic form

$$
a_{1}[u, u]=\int_{\Omega \backslash \Sigma} \sigma_{1} \nabla^{b} u \cdot \overline{\nabla^{b} u} d x+\int_{\Omega \backslash \Sigma} q g^{1 / 2}|u|^{2} d x,
$$

defined in the domain $\mathscr{D}\left(a_{1}\right)=H_{0, g}^{1}(\Omega)$ is closed. Moreover, the embedding $\mathscr{D}\left(a_{1}\right)$ $\hookrightarrow L_{g}^{2}(\Omega)$ is compact, and there is $C_{b}>1$ so that

$$
C_{b}^{-1}\|u\|_{H_{0, g}^{1}(\Omega)}^{2} \leq\|u\|_{g}^{2}+a_{1}[u, u] \leq C_{b}\|u\|_{H_{0, g}^{1}(\Omega)}^{2} .
$$

Proof. To prove the assertion, we need to show two facts. First, we need to prove that $a_{1}[u, u]<\infty$ for $u \in H_{0, g}^{1}(\Omega)$. Second, we have to prove the closedness of the form $a_{1}$ on $H_{0 . g}^{1}(\Omega)$.

We start with the fact [24] that the map $F_{*}$ is unitary from $L^{2}\left(M_{1}\right) \oplus L^{2}\left(M_{2}\right)$ to $L_{g}^{2}(\Omega)$ and from $H_{0}^{1}\left(M_{1}\right) \oplus H^{1}\left(M_{2}\right)$ to $H_{0, g}^{1}(\Omega)$, respectively. For $v=\left(v_{1}, v_{2}\right)$ and $u=F_{*} v$, we have

$$
\begin{array}{ll}
\left(v_{1}, v_{2}\right) \in L^{2}\left(M_{1}\right) \oplus L^{2}\left(M_{2}\right) & \text { iff } u \in L_{g}^{2}(\Omega), \\
\left(v_{1}, v_{2}\right) \in H_{0}^{1}\left(M_{1}\right) \oplus H^{1}\left(M_{2}\right) & \text { iff } u \in H_{0, g}^{1}(\Omega) .
\end{array}
$$

Hence, as $H^{1}\left(M_{j}\right), j=1,2$ is compactly embedded into $L^{2}\left(M_{j}\right)$, we see that the space $H_{g}^{1}(\Omega)$ is compactly embedded into $L_{g}^{2}(\Omega)$. 
Using definition (4) and the transformation rule for 1-forms, we see that

$$
\begin{gathered}
a_{1}[u, u]=\alpha[v, v]=\alpha^{1}\left[v_{1}, v_{1}\right]+\alpha^{2}\left[v_{2}, v_{2}\right] ; \\
\alpha^{1}\left[v_{1}, v_{1}\right]=\int_{M_{1}}\left|\nabla v_{1}+i \beta_{1} v_{1}\right|^{2} d x+\int_{M_{1}} \kappa_{1}\left|v_{1}\right|^{2} d x, \\
\alpha^{2}\left[v_{2}, v_{2}\right]=2 \int_{M_{2}}\left|\nabla v_{2}+i \beta_{2} v_{2}\right|^{2} d x+8 \int_{M_{2}} \kappa_{2}\left|v_{2}\right|^{2} d x .
\end{gathered}
$$

Here the 1-forms $\beta_{1}=\left.\beta\right|_{M_{1}}, \beta_{2}=\left.\beta\right|_{M_{2}}$, and functions $\kappa_{1}=\left.\kappa\right|_{M_{1}}, \kappa_{2}=\left.\kappa\right|_{M_{2}}$, are given by $b=F_{*} \beta, q=F_{*} \kappa$, that is,

$$
b_{j}(x)=\frac{\partial F^{k}}{\partial y^{j}}(y) \beta_{k}(y), \quad q(x)=\kappa(y), \quad x=F(y) \in \Omega \backslash \Sigma .
$$

It follows from (2) and (3) that $\beta_{2}, \kappa_{1}$ and $\kappa_{2}$ are bounded, but $\beta_{1}$ has a singularity at $x=0$, of the order $1 /|x|$, and

$$
\left|\beta_{1}(x)\right| \leq \frac{C\|b\|_{L^{\infty}}}{|x|}
$$

Consider $\alpha^{2}\left[v_{2}, v_{2}\right]$ as an unbounded non-negative quadratic form in $L^{2}\left(M_{2}\right)$, with domain $\mathscr{D}\left(\alpha^{2}\right)=H^{1}\left(M_{2}\right)$. Then $\alpha^{2}$ is closed. The quadratic form $\alpha^{1}\left[v_{1}, v_{1}\right]$ requires further analysis. We consider $\alpha^{1}\left[v_{1}, v_{1}\right]$ as an unbounded non-negative quadratic form in $L^{2}\left(M_{1}\right)$ having the domain

$$
\mathscr{D}\left(\alpha^{1}\right)=\left\{v_{1} \in L^{2}\left(M_{1}\right): \nabla v_{1}+i \beta_{1} v_{1} \in L^{2}\left(M_{1}\right),\left.v_{1}\right|_{\partial M_{1}}=0\right\} .
$$

Note that the condition $\nabla v_{1}+i \beta_{1} v_{1} \in L^{2}\left(M_{1}\right)$ implies that the trace $\left.v_{1}\right|_{\partial M_{1}}$ is well-defined. By [47] and [68], $C_{0}^{\infty}\left(M_{1}\right)$ is dense on $\mathscr{D}\left(\alpha^{1}\right)$ and $\alpha^{1}$ on $\mathscr{D}\left(\alpha^{1}\right)$ is a closed, non-negative quadratic form.

By Hardy's inequality [43], it follows from (15) that

$$
\left\|\beta_{1} v_{1}\right\|_{L^{2}\left(M_{1}\right)} \leq C\|b\|_{L^{\infty}}\left\|v_{1}\right\|_{H^{1}\left(M_{1}\right)}
$$

so that

$$
H_{0}^{1}\left(M_{1}\right) \subset \mathscr{D}\left(\alpha^{1}\right) \text { is dense. }
$$

Let $v_{1} \in \mathscr{D}\left(\alpha^{1}\right)$. Then, by [47], $\left|v_{1}\right| \in H_{0}^{1}\left(M_{1}\right)$. Using (17) again, we obtain

$$
\left\|\beta_{1} v_{1}\right\|_{L^{2}\left(M_{1}\right)}=\left\|\beta_{1}\left|v_{1}\right|\right\|_{L^{2}\left(M_{1}\right)}<\infty .
$$

Thus, by definition (16), this yields that $\nabla v_{1} \in L^{2}\left(M_{1}\right)$, and hence $v_{1} \in H_{0}^{1}\left(M_{1}\right)$. This shows that $\mathscr{D}\left(\alpha^{1}\right) \subset H_{0}^{1}\left(M_{1}\right)$ which, together with (18), implies that $\mathscr{D}\left(\alpha^{1}\right)=$ $H_{0}^{1}\left(M_{1}\right)$. Thus, the domain of the closed form $\alpha$ is $\mathcal{D}(\alpha)=H_{0}^{1}\left(M_{1}\right) \oplus H^{1}\left(M_{2}\right)$. Using the transformation rule (14) for $u=F_{*} v$ and (13) we conclude that $a_{1}[u, u]<$ $\infty$ for all $u \in H_{0, g}^{1}(\Omega)$ and that the quadratic form $a_{1}$ with domain $\mathscr{D}\left(a_{1}\right)=H_{0, g}^{1}(\Omega)$ is closed. The inequality (12) follows from this by the open mapping theorem. 
By the theory of quadratic forms [39], the closed quadratic form $a_{1}[u, u]$ defines an associated non-negative self-adjoint operator $\mathcal{A}_{1}$ in $L_{g}^{2}(\Omega)$, having domain

$$
\begin{aligned}
& \mathscr{D}\left(\mathcal{A}_{1}\right)=\left\{u \in H_{0, g}^{1}(\Omega): \text { there is } f \in L_{g}^{2}(\Omega)\right. \text { such that } \\
& \left.\qquad a_{1}[u, v]=(f, v)_{g} \text { for } v \in H_{0, g}^{1}(\Omega)\right\},
\end{aligned}
$$

that is, for $u \in \mathscr{D}\left(A_{1}\right)$ we have, for all $v \in H_{0, g}^{1}(\Omega)$,

$$
\int_{\Omega \backslash \Sigma} \sigma_{1} \nabla^{b} u \cdot \overline{\nabla^{b} v} d x+\int_{\Omega \backslash \Sigma} q g^{1 / 2} u \bar{v} d x=\int_{\Omega \backslash \Sigma} g^{1 / 2} f \bar{v} d x
$$

with $\mathcal{A}_{1} u=f \in L_{g}^{2}(\Omega)$. By Lemma 2.1, the spectrum of $\mathscr{A}_{1}$ consists of discrete eigenvalues with finite dimensional eigenspaces.

Observe that, formally integrating by parts in (20), we come to (10) with $h=$ 0 . To better understand the nature of the operator $\mathcal{A}_{1}$, we give also an alternative definition in the case when $b \in C\left(\Omega ; \mathbb{R}^{3}\right)$. In this case, we use the fact that the map $D_{\sigma}^{j}: \varphi \mapsto \sigma_{1}^{j k}\left(\partial_{k} \varphi+i b_{k} \varphi\right)$, defined initially for $\varphi \in C_{0}^{\infty}(\Omega)$, has a bounded extension

$$
D_{\sigma}^{j}: H_{0, g}^{1}(\Omega) \rightarrow \mathcal{M}\left(\Omega ; \mathbb{R}^{3}\right),
$$

where $\mathcal{M}(\Omega ; \mathbb{R})$ denotes the space of Borel measures on $\Omega$, cf. Lemma 3.2 in [24]. For $b \in C\left(\Omega ; \mathbb{R}^{3}\right)$, an equivalent definition of the operator $\mathcal{A}_{1}$ is then

$$
\begin{aligned}
& \mathcal{A}_{1}(u)=-g^{-1 / 2}\left(\partial_{j}+i b_{j}\right) D_{\sigma}^{j} u+q u, \\
& \mathscr{D}\left(\mathcal{A}_{1}\right)=\left\{u \in H_{0, g}^{1}(\Omega):\left(\partial_{j}+i b_{j}\right) D_{\sigma}^{j} u \in L^{2}\left(\Omega, g^{-1 / 2} d x\right)\right\} .
\end{aligned}
$$

2.2. Approximate cloaking by truncation. For any $1<R<2$, consider the nonsingular truncations of (10),

$$
-\nabla^{b} \cdot \sigma_{R} \nabla^{b} u+q g^{1 / 2} u-\lambda g^{1 / 2} u=0 \text { in } \Omega,\left.\quad u\right|_{\partial \Omega}=h,
$$

where $\sigma_{R}$ are measurable anisotropic conductivities in $\Omega$ satisfying

$$
\begin{gathered}
\left.\sigma_{R}\right|_{\Omega \backslash B(0,5 / 2)}=\gamma_{0},\left.\quad \sigma_{R}\right|_{B(0,1)}=2 \gamma_{0}, \\
\lim _{R \searrow 1} \sigma_{R}(x)=\sigma(x), \quad c_{1}(R-1) \gamma_{0} \leq \sigma_{R}(x) \leq c_{2} \gamma_{0}, \quad \text { for } x \in \Omega, \\
\sigma_{R_{2}}(x) \leq \sigma_{R_{1}}(x), \quad \text { for } R_{2} \geq R_{1},
\end{gathered}
$$

for some $c_{1} \leq 1 / 2, c_{2} \geq 2$. For instance, we can choose

$$
\sigma_{R}(x)= \begin{cases}F_{*} \gamma_{0}, & x \in \Omega \backslash \bar{B}(R), \\ 2 \gamma_{0}, & x \in B(R) .\end{cases}
$$


We note that, by smoothing the conductivities (24), it is possible to construct conductivities $\sigma_{R} \in C^{2}\left(\bar{\Omega} ; \mathbb{R}^{2}\right)$, which we use in Section 5, satisfying (23).

We denote the solution of (22) by $u=u_{R}^{h}$. Note that, for $b=0$ and $q=0$, eq. (22) is an acoustic equation with the mass density $\sigma_{R}^{-1}$ and the bulk modulus $g^{-1 / 2}$ with $k=\sqrt{\lambda}$; in the quantum mechanical setting, $\sigma_{R}$ corresponds to the inverse matrix of effective mass and $(q-\lambda) g^{1 / 2}$ to the potential. By abuse of notation, even for $b \neq 0$ and $q \neq 0$, we will refer to $\sigma_{R}$ as the inverse of the mass density.

Observe that, for each $R>1$, the inverse of the mass density $\sigma_{R}$ is nonsingular, i.e. is bounded from above and below with, however, the lower bound going to 0 as $R \searrow 1$. Moreover, for any $x \in \mathbb{R}^{3}$, the symmetric matrix valued function $R \mapsto \sigma_{R}(x)$ is increasing ${ }^{6}$ as a function of $R$, and therefore decreases as $R \searrow 1$. Nonsingular regularizations or truncations of singular ideal cloaks have previously been considered in [66], [26], and [42].

To motivate the treatment here, consider for $R>1$ the Dirichlet-to-Neumann (DN) map $\Lambda_{R}^{\lambda}: H^{1 / 2}(\partial \Omega) \rightarrow H^{-1 / 2}(\partial \Omega)$ that maps

$$
\Lambda_{R}^{\lambda}:\left.\left.u\right|_{\partial \Omega} \mapsto\left(\partial_{\nu} u+i v \cdot b u\right)\right|_{\partial \Omega},
$$

where $u$ solves (22). The DN map corresponds to the Dirichlet-to-Neumann quadratic form, which by abuse of notation is denoted also by $\Lambda_{R}^{\lambda}$,

$$
\Lambda_{R}^{\lambda}[h]=\int_{\partial \Omega}\left(\Lambda_{R}^{\lambda} h\right)(x) \overline{h(x)} d S(x),
$$

where we denote $h=\left.u\right|_{\partial \Omega} \in H^{1 / 2}(\partial \Omega)$; for $\lambda \leq 0$, the Dirichlet-to-Neumann form may be also represented as

$$
\Lambda_{R}^{\lambda}[h]=\inf \left(a_{R}[u, u]-\lambda\|u\|_{g}^{2}\right),
$$

where infimum is taken over all $u \in H^{1}(\Omega)$ with $\left.u\right|_{\partial \Omega}=h$. However, to treat general $\lambda$, and the general class of eq. (22), we will use the definition (31) below.

Returning to eq. (22), note that for $\lambda<0$ and $R>1$ the solution can be obtained from the minimization problem for the quadratic functional associated to the sesquilinear functional

$$
\begin{aligned}
a_{R}[u, v]= & \int_{\Omega} \sigma_{R}(x) \nabla^{b} u(x) \cdot \overline{\nabla^{b} v(x)} d x \\
& +\int_{\Omega} q(x) g^{1 / 2}(x) u(x) \overline{v(x)} d x .
\end{aligned}
$$

Moreover, we have

$$
u_{R}^{h}=\operatorname{argmin}\left(a_{R}[u, u]-\lambda\|u\|_{g}^{2}\right),
$$

\footnotetext{
${ }^{6}$ Note that, due to the behavior of the eigenvalues of $\sigma_{1}$, this simple but important monotonicity property fails in the 2D case, which we thus do not deal with. However, approximate cloaking for the Helmholtz equation in $2 \mathrm{D}$ has now been treated in [41] by other methods.
} 
where minimization is taken over $u \in H^{1}(\Omega)$ such that $\left.u\right|_{\partial \Omega}=h$. Observe that the DN form (27) is related to $a_{R}$ defined by (28).

Thus, the solution $u_{R}^{h}$ of the acoustic equation (22) with the Dirichlet boundary value $h$ is the minimizer of $a_{R}[u, u]-\lambda\|u\|_{g}^{2}$.

Now consider the quadratic form $a_{R}[u, u]$ with domain $u \in H_{0}^{1}(\Omega)$, and we prove that it is a closed unbounded form on $L_{g}^{2}(\Omega)$ in sense of Section VI.1.3 of [39].

Lemma 2.2. Let $R>1$ and consider the sesquilinear form given by (28), $(u, v) \mapsto$ $a_{R}[u, v]$ with $u, v \in H_{0}^{1}(\Omega)$, as an unbounded form on $L_{g}^{2}(\Omega)$ with the domain $\mathscr{D}\left(a_{R}\right)=H_{0}^{1}(\Omega)$. Then $a_{R}: L_{g}^{2}(\Omega) \times L_{g}^{2}(\Omega) \rightarrow \mathbb{C}$ is closed and positive definite unbounded sesquilinear form.

Proof. Clearly $a_{R}$ is non-negative. The fact that $a_{R}$ is closed on $\mathscr{D}_{R}=H_{0}^{1}(\Omega)$ follows from the same considerations as those in Lemma 2.1. Next we show that $\nabla^{b} u=0$ iff $u=0$ which, due to the compactness of the embedding $H_{0}^{1}(\Omega) \hookrightarrow L_{g}^{2}$, implies the positive-definiteness of $a_{R}$. Assume, on the contrary, that there is a nonzero $\psi \in H_{0}^{1}(\Omega)$ such that $\nabla^{b} \psi=0$ in $\Omega$. Continue $b$ and $\psi$ by 0 to $\mathbb{R}^{3} \backslash \Omega$. and also extend $\sigma_{R}$ to $\mathbb{R}^{3} \backslash \Omega$ as $\gamma_{0}=1$ in $\mathbb{R}^{3} \backslash B(3)$. Then $\psi \in H^{1}\left(\mathbb{R}^{3}\right)$ and $\nabla^{b} \psi=0$ in $\mathbb{R}^{3}$, and hence $\nabla^{b} \cdot \nabla^{b} \psi=0$ in $\mathbb{R}^{3} \backslash\{O\}$. Using unique continuation for elliptic equations with non-smooth first order terms, see [5] and [40], we deduce that $\psi=0$ in $\mathbb{R}^{3} \backslash\{O\}$.

As $\sigma_{R}$ is bounded from below, Lemma 2.2 implies that there is $c_{R}>0$ such that

$$
\int_{\Omega} \sigma_{R} \nabla^{b} u \cdot \overline{\nabla^{b} u} d x \geq c_{R}\|u\|_{L^{2}(\Omega)}^{2}, \quad u \in H_{0}^{1}(\Omega) .
$$

Similarly to (19), (20), the sesquilinear form $a_{R}$ defines a self-adjoint operator $\mathcal{A}_{R}$ in $L_{g}^{2}$ and, analogously to (21), we have

$$
\begin{gathered}
\mathcal{A}_{R}(u)=-g^{-1 / 2} \nabla^{b} \cdot\left(\sigma_{R} \nabla^{b} u\right)+q u, \\
\mathscr{D}\left(\mathcal{A}_{R}\right)=\left\{u \in H_{0}^{1}(\Omega): \nabla^{b} \cdot\left(\sigma_{R} \nabla^{b} u\right) \in L^{2}\left(\Omega, g^{-1 / 2} d x\right)\right\} .
\end{gathered}
$$

Using the operator $\mathcal{A}_{R}, R>1$ we see that, for $\lambda \notin \operatorname{spec}\left(\mathcal{A}_{R}\right)$,

$$
\begin{gathered}
\Lambda_{R}^{\lambda} h=\left.\left(\partial_{v}+i v \cdot b\right)\left(u_{0}+v_{R}^{\lambda}\right)\right|_{\partial \Omega}, \\
v_{R}^{\lambda}=\mathcal{R}_{R}(\lambda)\left(\nabla^{b} \cdot\left(\sigma_{R} \nabla^{b} u_{0}\right)-q u_{0}+\lambda u_{0}\right) .
\end{gathered}
$$

Here $u_{0} \in H^{1}(\Omega)$ with $\operatorname{supp}\left(u_{0}\right) \subset \Omega \backslash \overline{B(O, 2)}$ satisfies $\left.u_{0}\right|_{\partial \Omega}=h$, while $\mathcal{R}_{R}(\lambda)$ is the resolvent

$$
\mathcal{R}_{R}(\lambda)=\left(\mathcal{A}_{R}-\lambda I\right)^{-1}: H^{-1}(\Omega) \rightarrow L^{2}(\Omega) .
$$


We will use the notation (32) for $R=1$ as well as $R>1$, in which case we have $\mathcal{R}_{1}(\lambda): H_{g}^{-1}(\Omega) \rightarrow L_{g}^{2}(\Omega)$. Note that the right-hand side in (31) should be understood in the sense of the pairing $H^{-1 / 2}(\partial \Omega) \times H^{-1 / 2}(\partial \Omega)$,

$$
\int_{\partial \Omega}\left(\left(\partial_{v}+i v \cdot b\right)\left(u_{0}+v_{R}^{\lambda}\right)\right) \bar{\psi} d S=a_{R}\left[u_{0}+v_{R}^{\lambda}, \psi\right]-\lambda\left(u_{0}+v_{R}^{\lambda}, \psi\right)_{L_{g}^{2}},
$$

for all $\psi \in H^{1}(\Omega)$, and we again consider $a_{R}$ on the whole of $H^{1}(\Omega)$.

Next, consider the DN map in the ideal case $R=1$. Following [24], we say that $u$ is a finite energy solution of the boundary value problem (10) with $h \in H^{1 / 2}(\partial \Omega)$ and $f \in H_{g}^{-1}(\Omega)$, if $u \in H_{g}^{1}(\Omega)$ and

$$
a_{1}\left[u-u_{0}, \varphi\right]=-\int_{\Omega}\left(\sigma_{1} \nabla^{b} u_{0} \cdot \overline{\nabla^{b} \varphi}+\left(q g^{1 / 2} u_{0}-\lambda\right) g^{1 / 2} u-f\right) \bar{\varphi} d x,
$$

for every $\varphi \in H_{0, g}^{1}(\Omega)$. Here $H_{g}^{-1}(\Omega)$ is the dual space to $H_{0, g}^{1}(\Omega)$.

On the other hand,

$$
\begin{gathered}
-\left(\nabla+i \beta_{1}\right) \cdot\left(\nabla+i \beta_{1}\right) v_{1}+\kappa_{1} v_{1}-\lambda v_{1}=\tilde{f}_{1} \text { in } M_{1}, \\
\left.v_{1}\right|_{\partial M_{1}}=h,
\end{gathered}
$$

and

$$
\begin{gathered}
-\frac{1}{4}\left(\nabla+i \beta_{2}\right) \cdot\left(\nabla+i \beta_{2}\right) v_{2}+\kappa_{2} v_{2}-\lambda v_{2}=\tilde{f}_{2} \text { in } M_{2}, \\
\left.\left(\partial_{v}+i v \cdot \beta_{2}\right) v_{2}\right|_{\partial M_{2}}=0,
\end{gathered}
$$

are satisfied in the weak sense if

$$
\alpha\left[v-v_{0}, \psi\right]=-\sum_{j=1}^{2} \int_{M_{j}}\left(c_{j} \nabla^{\beta} v_{0} \cdot \overline{\nabla^{\beta} \psi}+\left(\kappa_{j} u_{0}-\lambda v-\tilde{f}_{j}\right) \bar{\psi}\right) d x
$$

for all $\psi \in H_{0}^{1}\left(M_{1}\right) \oplus H^{1}\left(M_{2}\right)$, where $c_{1}=1, c_{2}=1 / 4$. Here $v_{0} \in H^{1}\left(M_{1}\right) \oplus$ $H^{1}\left(M_{2}\right)$ is supported in $M_{1} \backslash \overline{B(2)}$ and satisfies $\left.v_{0}\right|_{\partial M_{1}}=h$.

Lemma 2.3. Let $h \in H^{1 / 2}(\partial \Omega)$ and $f \in H_{g}^{-1}(\Omega)$. A function $u \in H_{g}^{1}(\Omega)$ is a finite energy solution in the sense (33) of the boundary value problem (10) if and only if $v=\left(v_{1}, v_{2}\right) \in H^{1}\left(M_{1}\right) \oplus H^{1}\left(M_{2}\right), u=F_{*} v$ satisfies eq. (34) and (35) in the weak sense (36) with $f=F_{*}\left(\tilde{f}_{1}, \tilde{f}_{2}\right)$.

In particular, for any $\lambda \in \mathbb{R}$ the Cauchy data, on $\partial \Omega$, of solutions to (22) satisfy

$$
\begin{aligned}
& \left\{\left(\left.u\right|_{\partial \Omega},\left.\left(\partial_{v}+i v \cdot b\right) u\right|_{\partial \Omega}\right):-\nabla^{b} \cdot \sigma_{1} \nabla^{b} u+q g^{1 / 2} u=g^{1 / 2} \lambda u \text { in } \Omega\right\} \\
& \quad=\left\{\left(\left.v_{1}\right|_{\partial \Omega},\left.\left(\partial_{v}+i v \cdot \beta_{1}\right) v_{1}\right|_{\partial \Omega}\right):-\nabla^{\beta_{1}} \cdot \nabla^{\beta_{1}} v_{1}+\kappa_{1} v_{1}=\lambda v_{1} \text { in } \Omega\right\} \\
& \quad \subset H^{1 / 2}(\partial \Omega) \times H^{-1 / 2}(\partial \Omega)
\end{aligned}
$$


Proof. By Lemma 2.1 and formulae (13) and (14) we see that $u \in \mathscr{D}\left(a_{1}\right)$ if and only if $u=F_{*} v, v=\left(v_{1}, v_{2}\right) \in \mathscr{D}(\alpha)$, and

$$
a_{1}[\tilde{\psi}, \tilde{\varphi}]=\alpha[\psi, \varphi]
$$

for $\varphi, \psi \in \mathscr{D}(\alpha), \tilde{\varphi}=F_{*} \varphi, \tilde{\psi}=F_{*} \psi$.

Let $v_{0} \in H^{1}\left(M_{1}\right) \oplus H^{1}\left(M_{2}\right)$ be supported in $M_{1} \backslash \overline{B(2)}$ and satisfy $\left.v_{0}\right|_{\partial M_{1}}=h$, and $u_{0} \in H^{1}(\Omega)$ be such that $u_{0}=F_{*} v_{0}$. Using formula (38) with $\tilde{\psi}=u-u_{0}$ and $\psi=v-v_{0}$, we see that $u \in H_{g}^{1}(\Omega)$ is a finite energy solution if and only if $v=\left(v_{1}, v_{2}\right) \in H^{1}\left(M_{1}\right) \oplus H^{1}\left(M_{2}\right)$, and setting $u=F_{*} v$, satisfies (36) for all $\varphi \in H_{0}^{1}\left(M_{1}\right) \oplus H^{1}\left(M_{2}\right)$, that is, $v$ satisfies eq. (34) and (35) in the weak sense.

Assume next that $\lambda \notin \operatorname{spec}\left(\mathcal{A}_{1}\right)$. Then the solution to eq. (10), in the sense of definition (36), may be found in terms of the resolvent $\mathcal{R}_{1}(\lambda)$ of $\mathcal{A}_{1}$, cf. (32). Indeed, comparing (19), (20) with eq. (33), we see that its solution $u$ has the form

$$
\begin{gathered}
u=u_{0}+\mathcal{R}_{1}(\lambda)\left(f+\nabla^{b} \cdot \sigma_{1} \nabla^{b} u_{0}-q u_{0}+\lambda u_{0}\right), \\
\left.u_{0}\right|_{\partial \Omega}=h, \quad \operatorname{supp}\left(u_{0}\right) \subset \Omega \backslash \overline{B(2),}
\end{gathered}
$$

at least when $f \in L_{g}^{2}(\Omega)$ and $h \in H^{3 / 2}(\partial \Omega)$ so that $u_{0} \in H^{2}(\Omega)$. Since $\mathscr{D}\left(a_{1}\right)=$ $\mathscr{D}\left(\mathcal{A}_{1}^{1 / 2}\right)=H_{0, g}^{1}(\Omega)$, we see that $H_{g}^{-1}(\Omega)=\mathscr{D}\left(\mathcal{A}_{1}^{-1 / 2}\right)$. Therefore, the operator $\mathcal{R}_{1}(\lambda)$ can be extended by continuity to a bounded operator from $H_{g}^{-1}(\Omega)$ onto $H_{0, g}^{1}(\Omega)$. This makes it possible to generalize (39) for all $u_{0} \in H_{g}^{1}(\Omega)$, i.e. $h \in$ $H^{1 / 2}(\partial \Omega)$, and $f \in H_{g}^{-1}(\Omega)$. Observe that the right-hand side in (37) is related to the unbounded selfadjoint operator $A_{\text {out }}$ in $L^{2}(\Omega), \mathscr{D}\left(A_{\text {out }}\right) \subset H_{0}^{1}(\Omega)$, associated with the form $\alpha_{1}$, see (14) where we use $\Omega=M_{1}$. More precisely, this operator is the unbounded selfadjoint operator in $L^{2}(\Omega)$ given by

$$
\begin{gathered}
A_{\text {out }}=-\nabla^{\beta_{1}} \cdot \nabla^{\beta_{1}}+\kappa_{1}, \\
\mathscr{D}\left(A_{\text {out }}\right)=\left\{v_{1} \in H^{1}(\Omega): \nabla^{\beta_{1}} \cdot \nabla^{\beta_{1}} v_{1} \in L^{2}(\Omega),\left.v_{1}\right|_{\partial \Omega}=0\right\} ;
\end{gathered}
$$

Moreover, when $b \in C^{1}\left(\bar{\Omega} ; \mathbb{R}^{3}\right)$, the selfajdoint operator associated to the form $\alpha_{2}$ on $B(1)=M_{2}$ is the operator

$$
\begin{gathered}
A_{\text {in }}=-\frac{1}{4} \nabla^{\beta_{2}} \cdot \nabla^{\beta_{2}}+\kappa_{2}, \\
\mathscr{D}\left(A_{\text {in }}\right)=\left\{v_{2} \in H^{2}(B(1)):\left.\partial_{\nu} v\right|_{\partial B(1)}=0\right\} .
\end{gathered}
$$

When $\lambda \notin \operatorname{spec}\left(A_{\text {out }}\right)$, the set (37) coincides with the graph of the DN-map

$$
\Lambda_{\text {out }}^{\lambda}:\left.\left.v_{1}\right|_{\partial \Omega} \mapsto\left(\partial_{\nu}+i \nu \cdot \beta_{1}\right) v_{1}\right|_{\partial \Omega},
$$

where $v_{1}$ solves eq. (34) with $\tilde{f}_{1}=0$. 
Note that if $b=0, q=0$, then $\lambda=0$ is an eigenvalue of $\mathcal{A}_{1}$ with the corresponding eigenfunctions of the form (cf. [35] and [24])

$$
u(x)=\left\{\begin{array}{ll}
0 & \text { for } x \in \Omega \backslash \bar{B}(1), \\
c_{0} & \text { for } \quad x \in B(1),
\end{array} \quad c_{0} \neq 0 .\right.
$$

However, as follows from Lemma 2.3 even, in this case the Cauchy data on $\partial \Omega$ of solutions of the eq. (10) with $\lambda=0$ coincide with the Cauchy data of the solutions of $\Delta v_{1}=0$ on $\partial M_{1}=\partial \Omega$.

2.3. $\Gamma$-convergence and spectral convergence. In this section we establish $\Gamma$-convergence and spectral convergence results for $a_{R}$ as $R \searrow 1$. To that end, following the comment in the previous section, observe that, since $\sigma_{R_{1}} \geq \sigma_{R_{2}}$ for $R_{1} \geq R_{2}$, one thus has

$$
a_{R_{1}}[v, v] \geq a_{R_{2}}[v, v], \quad v \in H^{1}(\Omega), \quad \text { if } R_{1} \geq R_{2}
$$

This implies that $R \mapsto a_{R}[v, v]$ is decreasing as $R \searrow 1$. For $R>1$, consider non-linear (quadratic) functionals $a_{R}: L_{g}^{2}(\Omega) \mapsto \mathbb{R}^{+}=\mathbb{R} \cup\{+\infty\}$,

$$
a_{R}(v)= \begin{cases}a_{R}[v, v] & \text { when } v \in H_{0}^{1}(\Omega) \\ \infty & \text { otherwise }\end{cases}
$$

For the ideal cloak, i.e. $R=1$, define

$$
a_{1}(v)= \begin{cases}a_{1}[v, v] & \text { when } v \in H_{0, g}^{1}(\Omega) \\ \infty & \text { otherwise }\end{cases}
$$

We will make extensive use of De Giorgi's $\Gamma$-convergence, see, e.g., [4] and [18].

Definition 2.4. Let $\left\{J_{R}: 1 \leq R \leq 2\right\}$ be a family of functionals on a Hilbert space $\mathcal{H}$. We say that the $J_{R} \Gamma$-converge to $J_{1}$, or $J_{1}=\Gamma-\lim _{R \searrow 1} J_{R}$ on $\mathscr{H}$, if

(i) for every $v \in \mathscr{H}$, and all sequences $v_{R}$ converging to $v$ in $\mathscr{H}$ as $R \searrow 1$, $J_{1}(v) \leq \liminf \operatorname{in}_{R \backslash 1} J_{R}\left(v_{R}\right)$ and

(ii) for every $w \in \mathscr{H}$ there exists a sequence $w_{R}$ converging to $w$ in $\mathscr{H}$ such that $J_{1}(w) \geq \lim \sup _{R \searrow 1} J_{R}\left(w_{R}\right)$.

Lemma 2.5. The functionals $a_{R} \Gamma$-converge to $a_{1}$ as $R \searrow 1$,

$$
\Gamma-\lim a_{R}=a_{1} \quad \text { on } L_{g}^{2}(\Omega)
$$


Proof. By Lemma 2.1, $H_{0, g}^{1}(\Omega)$ is a Hilbert space when endowed with the norm $\left(\|u\|_{L_{g}^{2}}^{2}+a_{1}(u)\right)^{1 / 2}$. Since the functionals $a_{\sigma_{R}}$ are pointwise decreasing as $R \searrow 1$, it follows from Proposition 5.7 in [18] that the functionals $a_{R} \Gamma$-converge on $L_{g}^{2}$ to

$$
\tilde{a}_{1}=\operatorname{sc}^{-} G
$$

that is the lower semicontinuous envelope of $G$ (see Definition 3.1 in [18]), where $G: L_{g}^{2} \rightarrow \mathbb{R}^{+}=\mathbb{R} \cup\{+\infty\}$ is defined by

$$
G(v)= \begin{cases}\int_{\Omega}\left(\sigma_{1} \nabla^{b} u \cdot \overline{\nabla^{b} u} d x+q g^{1 / 2}|u|^{2}\right) d x & \text { for } u \in H_{0}^{1}(\Omega) \\ \infty & \text { otherwise }\end{cases}
$$

By Proposition 11.10 in [18], the function $\tilde{a}_{1}: L_{g}^{2}(\Omega) \rightarrow \mathbb{R} \cup\{\infty\}$ is a quadratic form. Moreover, by Proposition 12.16 in [18], its domain

$$
\mathscr{D}\left(\tilde{a}_{1}\right)=\left\{u \in L_{g}^{2}(\Omega): \tilde{a}_{1}(u)<\infty\right\},
$$

endowed with the norm $\left(\|u\|_{g}^{2}+\tilde{a}_{1}(u)\right)^{1 / 2}$, is a Hilbert space.

Now $H_{0}^{1}(\Omega)$ is contained in both $\mathscr{D}\left(\tilde{a}_{1}\right)$ and $\mathscr{D}\left(a_{1}\right)=H_{0, g}^{1}(\Omega)$, and the norms of these Hilbert spaces coincide on $H_{0}^{1}(\Omega)$. Moreover, by the proof of Lemma 3.3 in [24], $H_{0}^{1}(\Omega)$ is dense in $\mathscr{D}\left(a_{1}\right)$. Thus $\mathscr{D}\left(a_{1}\right) \subset \mathscr{D}\left(\tilde{a}_{1}\right)$.

On the other hand, as $G(v) \geq a_{1}(v)$ for all $v \in L_{g}^{2}(\Omega)$ and $a_{1}$ is lower semicontinuous (see Proposition 2.16 in [18]), it follows that the lower semicontinuous envelope $\tilde{a}_{1}$ of $G$ also satisfies $\tilde{a}_{1}(v) \geq a_{1}(v)$. Hence $\mathcal{D}\left(\tilde{a}_{1}\right) \subset \mathcal{D}\left(a_{1}\right)$. Thus, $\mathscr{D}\left(\tilde{a}_{1}\right)=\mathscr{D}\left(a_{1}\right)$.

Let us next consider the resolvent $\mathcal{R}_{R}(\lambda)$ for $\lambda<0$.

Lemma 2.6. For any $\lambda<0$, the resolvents $\mathcal{R}_{R}(\lambda), R>1$, strongly converge on $L_{g}^{2}(\Omega)$ to $\mathcal{R}_{1}(\lambda)$, i.e., for any $f \in L_{g}^{2}(\Omega)$,

$$
\lim _{R \searrow 1} \mathcal{R}_{R}(\lambda) f=\mathcal{R}_{1}(\lambda) f
$$

strongly in $L_{g}^{2}(\Omega)$ and weakly in $H_{0, g}^{1}(\Omega)$.

Proof. The quadratic forms $a_{R}(u)-\lambda\|u\|_{g}^{2}, R \geq 1$ in the Hilbert space $L_{g}^{2}(\Omega)$ are associated to the unbounded selfadjoint operators $\mathcal{A}_{R}-\lambda I$. Thus we can use Theorem 13.6 in [18] to show that the resolvents $\left(\mathcal{A}_{R}-\lambda I\right)^{-1}$ satisfy $(50)$ in $L_{g}^{2}(\Omega)$. Indeed, to show the strong convergence $(50)$ in $L_{g}^{2}(\Omega)$ it is sufficient to prove the following three properties:

$$
a_{R}: L_{g}^{2}(\Omega) \rightarrow \mathbb{R} \cup\{\infty\} \text { are lower semicontinuous; }
$$




$$
\begin{gathered}
\Gamma-\lim _{R \searrow 1} a_{R}=a_{1} \quad \text { on } L_{g}^{2}(\Omega) \\
a_{1}(u)-\lambda\|u\|_{g}^{2} \leq \liminf _{R \searrow 1}\left(a_{R}\left(u_{R}\right)-\lambda\left\|u_{R}\right\|_{g}^{2}\right) \quad \text { as } u_{R} \rightarrow u \operatorname{in} L_{g}^{2}(\Omega),
\end{gathered}
$$

where $\rightarrow$ denotes weak convergence in $L_{g}^{2}(\Omega)$.

Clearly, the quadratic forms $a_{R}(u)-\lambda\|u\|_{g}^{2}$ are lower semicontinuous on $L_{g}^{2}(\Omega)$, see proof of Lemma 2.5. By Lemma 2.5, the quadratic forms $a_{R} \Gamma$-converge to $a_{1}$. Thus (51) and (52) are valid. To see (53), it suffices to consider the case when $u_{R} \rightarrow u$ in $L_{g}^{2}$ and

$$
\liminf _{R \searrow 1}\left(a_{R}\left(u_{R}\right)-\lambda\left\|u_{R}\right\|_{g}^{2}\right)<\infty
$$

Next we consider a sequence $R^{k} \searrow 1$ such that

$$
\liminf _{R \searrow 1}\left(a_{R}\left(u_{R}\right)-\lambda\left\|u_{R}\right\|_{g}^{2}\right)=\lim _{k \rightarrow \infty}\left(a^{k}\left(u^{k}\right)-\lambda\left\|u^{k}\right\|_{g}^{2}\right)
$$

where $a^{k}=a_{R^{k}}$ and $u^{k}=u_{R^{k}} \in H_{0}^{1}(\Omega)$. Since $u^{k}$ converges weakly in $L_{g}^{2}$, the norms $\left\|u^{k}\right\|_{g}$ are uniformly bounded. Since also

$$
a_{1}\left(u^{k}\right) \leq a^{k}\left(u^{k}\right),
$$

we see that the sequence $u^{k}$ is uniformly bounded in $H_{g}^{1}(\Omega)$. Let us now choose a subsequence of $u^{k}$ ( still denoted by $u^{k}$ ) which converges weakly to $u$ in $H_{0, g}^{1}(\Omega)$. As the embedding $H_{g}^{1} \hookrightarrow L_{g}^{2}$ is compact, this sequence converges strongly in $L_{g}^{2}(\Omega)$. Using the weak convergence in $H_{0, g}^{1}(\Omega)$, we see that

$$
a_{1}(u)-\lambda\|u\|_{g}^{2}=\lim _{k \rightarrow \infty} \int_{\Omega}\left(\sigma_{1} \nabla^{b} u^{k} \cdot \overline{\nabla^{b} u}+g^{1 / 2} q u^{k} \bar{u}-\lambda g^{1 / 2} u^{k} \bar{u}\right) d x .
$$

By the Cauchy-Schwarz inequality,

$$
\begin{aligned}
& \left|\int_{\Omega}\left(\sigma_{1} \nabla^{b} u^{k} \cdot \overline{\nabla^{b} u}+g^{1 / 2} q u^{k} \bar{u}-\lambda g^{1 / 2} u^{k} \bar{u}\right) d x\right| \\
& \leq\left(\int_{\Omega}\left(\sigma_{1} \nabla^{b} u^{k} \cdot \overline{\nabla^{b} u^{k}}+g^{1 / 2} q\left|u^{k}\right|^{2}-\lambda g^{1 / 2}\left|u^{k}\right|^{2}\right) d x\right)^{\frac{1}{2}} \\
& \quad \cdot\left(\int_{\Omega}\left(\sigma_{1} \nabla^{b} u \cdot \overline{\nabla^{b} u}+g^{1 / 2} q|u|^{2}-\lambda g^{1 / 2}|u|^{2}\right) d x\right)^{\frac{1}{2}} \cdot
\end{aligned}
$$

Using (54) and (55), this implies that

$$
\begin{aligned}
a_{1}(u)-\lambda\|u\|_{g}^{2} & \leq \liminf _{k \rightarrow \infty}\left(a_{1}\left(u^{k}\right)-\lambda\left\|u^{k}\right\|_{g}^{2}\right)^{1 / 2} \cdot\left(a_{1}(u)-\lambda\|u\|_{g}^{2}\right)^{1 / 2} \\
& \leq \liminf _{k \rightarrow \infty}\left(a_{k}\left(u^{k}\right)-\lambda\left\|u^{k}\right\|_{g}^{2}\right)^{1 / 2} \cdot\left(a_{1}(u)-\lambda\|u\|_{g}^{2}\right)^{1 / 2}
\end{aligned}
$$


The desired inequality (53) follows immediately, proving $(50)$ in $L_{g}^{2}(\Omega)$.

Finally, to prove that (50) holds weakly in $H_{0, g}^{1}(\Omega)$, observe that, since $\sigma_{R} \geq \sigma_{1}$,

$$
\int_{\Omega}\left(\sigma_{R} \nabla^{b} v \cdot \overline{\nabla^{b} v}+g^{1 / 2} q|v|^{2}\right) d x \geq \int_{\Omega}\left(\sigma_{1} \nabla^{b} v \cdot \overline{\nabla^{b} v}+g^{1 / 2} q|v|^{2}\right) d x
$$

for $v \in H_{0}^{1}(\Omega)$. On the other hand, denoting $u_{R}=\mathcal{R}_{R}(\lambda) f$ and using $\lambda \leq 0$, we have

$$
\int_{\Omega}\left(\sigma_{R} \nabla^{b} u_{R} \cdot \overline{\nabla^{b} u_{R}}+g^{1 / 2} q\left|u_{R}\right|^{2}\right) d x \leq\|f\|_{g}\left\|u_{R}\right\|_{g} .
$$

The above two inequalities, together with the strong convergence (50) in $L_{g}^{2}(\Omega)$, show that the $\left\|u_{R}\right\|_{H_{g}^{1}}$ are uniformly bounded. Thus, if weak convergence (50) in $H_{0, g}^{1}(\Omega)$ is not valid, there is a $v \neq u$ and a subsequence $R_{n} \searrow 1$ such that $u_{R_{n}} \rightarrow v$ in $H_{g}^{1}$. Then $u_{R_{n}} \rightarrow v$ in $L_{g}^{2}(\Omega)$, which is in contradiction with the strong convergence (50) in $L_{g}^{2}(\Omega)$. Thus (50) holds weakly in $H_{0, g}^{1}(\Omega)$.

In some applications, e.g. dealing with scattering of plane waves $e^{i<k, x>}, k \in \mathbb{R}^{3}$, by the cloaking device, the main interest concerns not values $\lambda<0$, but rather $\lambda=|k|^{2}>0$. To analyze this case, let us first consider the behavior of the spectra $\operatorname{spec}\left(\mathcal{A}_{R}\right)$ as $R \searrow 1$.

Lemma 2.7. Let $K$ be a compact set with $K \cap \operatorname{spec}\left(\mathcal{A}_{1}\right)=\emptyset$. Then, for $R$ sufficiently close to $1, K \cap \operatorname{spec}\left(\mathcal{A}_{R}\right)=\emptyset$.

Proof. It suffices to consider $K=[a, b] \subset \mathbb{R}$. Assume, to the contrary, the existence of a sequence $R_{n} \searrow 1, \mu_{n} \in[a, b]$, and functions $u_{n} \in H_{0}^{1} \subset H_{0, g}^{1},\left\|u_{n}\right\|_{g}=1$, such that

$$
\mathcal{A}_{R_{n}} u_{n}=\mu_{n} u_{n}
$$

Then,

$$
\int_{\Omega}\left(\sigma_{R_{n}} \nabla^{b} u_{n} \cdot \overline{\nabla^{b} u_{n}}+g^{1 / 2} q\left|u_{n}\right|^{2}\right) d x=\mu_{n} \int_{\Omega} g^{1 / 2}\left|u_{n}\right|^{2} d x=\mu_{n} \leq b .
$$

Therefore, as $\sigma_{R} \geq \sigma_{1}$ and $q \geq 0$, this implies $\left\|u_{n}\right\|_{H_{g}^{1}}^{2} \leq b+1$. Thus there exists a subsequence of $u_{n}$ and $\mu_{n}$, which we relabel as the original sequence, $u \in H_{0, g}^{1}$ and $\mu \in[a, b]$, such that

$$
u_{n} \rightarrow u \text { weakly in } H_{g}^{1}, \quad u_{n} \rightarrow u \text { strongly in } L_{g}^{2}, \quad \text { and } \mu_{n} \rightarrow \mu
$$

as $n \rightarrow \infty$. Thus, in particular, $\|u\|_{g}=1$. 
Taking e.g. $\lambda=-1$ in Lemma 2.6 , we know that $\mathcal{R}_{R}(-1) \rightarrow \mathcal{R}_{1}(-1)$ as $R \searrow 1$ in the strong operator topology on $L_{g}^{2}$. Consider $\mathcal{R}_{1}(-1) u$; then

$$
\mathcal{R}_{1}(-1) u=\lim _{n \rightarrow \infty} \mathcal{R}_{R_{n}}(-1) u=\lim _{n \rightarrow \infty} \mathcal{R}_{R_{n}}(-1) u_{n} \text { in } L_{g}^{2}(\Omega)
$$

where, in the last step we have used fact that $\mathcal{A}$ are non-negative operators yielding the estimate $\left\|\mathcal{R}_{R_{n}}(-1)\right\|_{L_{g}^{2} \rightarrow L_{g}^{2}} \leq 1$.

However, taking into account (57),

$$
\mathcal{R}_{R_{n}}(-1) u_{n}=\frac{1}{1+\mu_{n}} u_{n} \rightarrow \frac{1}{1+\mu} u \text { in } L_{g}^{2}(\Omega) \text { as } n \rightarrow \infty \text {. }
$$

Thus, $\mathcal{R}_{1}(-1) u=(1+\mu)^{-1} u$ with $\|u\|_{L_{g}^{2}}=1$, implying that $\mu \in \operatorname{spec}\left(\mathcal{A}_{1}\right)$ with $u$ being an associated eigenfunction. This contradiction proves the statement.

Lemma 2.8. Let $K \subset \mathbb{C}$ be compact subset such that $K \cap \operatorname{spec}\left(\mathcal{A}_{1}\right)=\emptyset$. Then,for any $f \in L_{g}^{2}(\Omega)$ and $\lambda \in K$,

$$
\lim _{R \searrow 1} \mathcal{R}_{R}(\lambda) f=\mathcal{R}(\lambda) f
$$

strongly in $H_{0, g}^{1}(\Omega)$, and the convergence is uniform for $\lambda \in K$.

Proof. Let $\delta>0$ satisfies

$$
K_{\delta} \cap \operatorname{spec}\left(\mathcal{A}_{1}\right)=\emptyset, \quad K_{\delta}=\{z \in \mathbb{C}: \operatorname{dist}(z, K) \leq \delta\} .
$$

It then follows from Lemma 2.7 that, for $R$ sufficiently close to $1, K_{\delta / 2} \cap \operatorname{spec}\left(\mathcal{A}_{R}\right)=$ $\emptyset$. As all $\mathcal{A}_{R}, R \geq 1$ are self-adjoint in $L_{g}^{2}(\Omega)$, we see that, for $R$ sufficiently close to 1 ,

$$
\left\|\mathcal{R}_{R}(\lambda)\right\|_{L_{g}^{2} \rightarrow L_{g}^{2}} \leq \frac{2}{\delta}, \quad \text { when } \lambda \in K
$$

This implies that $\mathbb{C} \backslash \operatorname{spec}\left(\mathcal{A}_{1}\right)=\Delta_{b}$, where $\Delta_{b}$ denotes the region of boundedness for the family of operators $\mathcal{A}_{R}, R \geq 1$, i.e. the set of those $\lambda \in \mathbb{C}$ for which the norms $\left\|\left(\mathcal{A}_{R}-\lambda\right)^{-1}\right\|_{L_{g}^{2} \rightarrow L_{g}^{2}}$ are bounded by some constant $C_{\lambda}>0$ for all $R>1$, see Section VIII.1.1 in [39]. As $\operatorname{spec}\left(\mathcal{A}_{1}\right)$ is countable, $\Delta_{b}$ is connected. On the other hand, by Lemma 2.6, $\mathbb{R}_{-} \subset \Delta_{S}$, where $\Delta_{S}$ is the region of strong convergence for the above family, i.e. the set of $\lambda \in \mathbb{C}$ such that for $f \in L_{g}^{2}(\Omega)$

$$
\lim _{R \rightarrow 1} \mathcal{R}_{R}(\lambda) f=\mathcal{R}_{1}(\lambda) f \quad \text { in } L_{g}^{2}(\Omega)
$$

Therefore, by Theorem VIII.1.2 in [39],

$$
\Delta_{s}=\Delta_{b}=\mathbb{C} \backslash \operatorname{spec}\left(\mathscr{A}_{1}\right) \text {. }
$$


By Lemma 3 in [24], $H_{0}^{1}(\Omega)$ is dense in $H_{0, g}^{1}(\Omega)=\mathscr{D}\left(a_{1}\right)$. Thus $H_{0}^{1}(\Omega)$ is a core of the quadratic form $a_{1}[\cdot, \cdot]$. Now $H_{0}^{1}(\Omega)=\mathscr{D}\left(a_{R}\right)$, for $R>1$ and $a_{R}$ are monotonically increasing with $R \geq 1$ on $H_{0}^{1}(\Omega)$. Thus it follows from Theorem VIII.3.6 in [39], that

$$
\lim _{R \searrow 1} a_{1}\left(\mathcal{R}_{R}(\lambda) f-\mathcal{R}_{1}(\lambda) f\right)=0,
$$

uniformly for $\lambda \in K$ where $K$ is an arbitrary compact subset of $\Delta_{s}$. By Lemma 2.1, the desired convergence (58) now follows from (59)-(61).

Let $\mu \notin \operatorname{spec}\left(\mathcal{A}_{1}\right)$. It follows from Lemma 2.7 that $\mu \notin \operatorname{spec}\left(\mathcal{A}_{R}\right)$ for $R>1$ sufficiently close to 1 . For $\mu \notin \operatorname{spec}\left(\mathcal{A}_{R}\right)$, we denote by $N_{R}(\mu)$ the subspace of $L_{g}^{2}(\Omega)$ spanned by the eigenfunctions of $\mathcal{A}_{R}$ with eigenvalues $\lambda_{j}<\mu$. We also denote by $P_{R}^{\mu}$ the orthoprojectors in $L_{g}^{2}(\Omega)$ onto $N_{R}(\mu)$. By Theorem III.6.17 in [39], these (Riesz) projectors $P_{R}$ have the representation

$$
P_{R}^{\mu} u=\frac{1}{2 \pi i} \int_{\Gamma}\left(\mathcal{A}_{R}-z\right)^{-1} u d z,
$$

where the contour $\Gamma \subset \mathbb{C}$ surrounds all the eigenvalues $\lambda_{j}$ of $\mathcal{A}_{R}$ satisfying $\lambda_{j}<\mu$ and only those.

Lemma 2.9. Let $\mu \notin \operatorname{spec}\left(\mathcal{A}_{1}\right)$. For $R$ sufficiently close to 1

$$
\operatorname{dim}\left(N_{R}(\mu)\right)=\operatorname{dim}\left(N_{1}(\mu)\right) .
$$

Moreover,

$$
\lim _{R \searrow 1}\left\|P_{R}^{\mu}-P_{1}^{\mu}\right\|_{L_{g}^{2} \rightarrow L_{g}^{2}}=0
$$

Proof. Recall that $\mathscr{D}\left(a_{R}\right)=H_{0}^{1}(\Omega)$ are independent of $R>1, H_{0}^{1}(\Omega) \subset \mathscr{D}\left(a_{1}\right)=$ $H_{0, g}^{1}(\Omega)$, and $a_{R}[u, u]$ are decreasing, as $R \searrow 1$, for all $u \in \mathscr{D}\left(a_{R}\right)$. Thus the identity (63) follows directly by Theorem VIII.3.15 in [39].

Using representation (62) and Lemma 2.8, we see that

$$
\lim _{R \searrow 1} P_{R}^{\mu}=P_{1}^{\mu} \quad \text { strongly in } L_{g}^{2}(\Omega) .
$$

As $P_{R}^{\mu}$ and $P_{1}^{\mu}$ are orthoprojectors, this and (63) yields (64) by [39] (see Lemmas VIII.1.23 and VIII.1.24).

We remark that in the course of this paper we need a number of results concerning convergence of orthoprojectors that appear similar to (64), e.g., (78), (114) and (133), but these are for different operators or with respect to different operator norms and require separate proofs. 


\subsection{Approximating the singular bulk modulus $\boldsymbol{g}^{-1 / 2}$ by nonsingular densities.} Above, in the operator $\mathcal{A}_{R}=-g^{-1 / 2} \nabla^{b} \cdot \sigma_{R} \nabla^{b}+q$, there appears the determinant $g$ of the metric (also denoted $g$ !), which vanishes at the cloaking surface $\Sigma$. We now consider how to approximate the scalar function $g$ by functions $g_{m}$ that are bounded from below with positive constants. To this end, we introduce the functions

$$
g_{m}(x)=\max (g(x), 1 / m), \quad m \in \mathbb{Z}_{+} .
$$

Then $L^{2}\left(\Omega, g_{m}^{1 / 2} d x\right) \subset L_{g}^{2}(\Omega)$ and

$$
\|f\|_{g} \leq\|f\|_{L^{2}\left(\Omega, g_{m}^{1 / 2} d x\right)}, \text { for } f \in L^{2}\left(\Omega, g_{m}^{1 / 2} d x\right) .
$$

The multiplication map $f \mapsto g^{1 / 2} f$ is unitary from $L_{g}^{2}=L^{2}\left(\Omega, g^{1 / 2} d x\right)$ onto $L^{2}\left(\Omega, g^{-1 / 2} d x\right)$. Note that $L^{2}\left(\Omega, g^{-1 / 2} d x\right) \subset L^{2}(\Omega) \subset L_{g}^{2}(\Omega)$. Next we will consider operators $g^{1 / 2} \mathcal{A}_{R} u$. For $f \in L_{g}^{2}(\Omega)$, we have

$$
\left(\mathcal{A}_{R}-\lambda\right) u=f
$$

where both sides are in $L_{g}^{2}(\Omega)$, if and only if $u$ is a solution to the acoustic equation with mass density tensor $\sigma_{R}^{-1}$, bulk modulus $g^{-1 / 2}$, and potential $q g^{1 / 2}$ ),

$$
\left(g^{1 / 2} \mathcal{A}_{R}-\lambda g^{1 / 2}\right) u=F,
$$

where $F=g^{1 / 2} f \in L^{2}\left(\Omega, g^{-\frac{1}{2}} d x\right)$. By the above considerations, we have for $f \in L_{g}^{2}(\Omega)$

$$
\left(\mathcal{A}_{R}-\lambda\right)^{-1} f=\left(g^{1 / 2} \mathcal{A}_{R}-\lambda g^{1 / 2}\right)^{-1}\left(g^{1 / 2} f\right)
$$

Later in this section we keep $R>1$ fixed. Define an unbounded selfadjoint operator $\mathcal{B}_{R}$ in $L^{2}(\Omega)$, having the same differential expression as the operator $g^{1 / 2} \mathcal{A}_{R}$, but with different domain

$$
\begin{gathered}
\mathcal{B}_{R} u=-\nabla^{b} \cdot\left(\sigma_{R} \nabla^{b} u\right)+g^{1 / 2} q u \\
\mathcal{D}\left(\mathscr{B}_{R}\right)=\left\{u \in H_{0}^{1}(\Omega): \nabla^{b} \cdot\left(\sigma_{R} \nabla^{b} u\right) \in L^{2}(\Omega)\right\} .
\end{gathered}
$$

Since $\mathscr{D}\left(\mathscr{A}_{R}\right) \subset H_{0}^{1}(\Omega)$, see $(30), \mathscr{B}_{R}$ is an extension of $g^{1 / 2} \mathcal{A}_{R}$ and, in particular, $\nabla^{b} \cdot\left(\sigma_{R} \nabla^{b} u\right) \in L^{2}\left(\Omega, g^{-1 / 2} d x\right)$ for $u \in \mathcal{D}\left(\mathcal{A}_{R}\right)$; however, $\nabla^{b} \cdot\left(\sigma_{R} \nabla^{b} u\right) \in L^{2}(\Omega)$ for $u \in \mathscr{D}\left(\mathscr{B}_{R}\right)$. Note that, by (69),

$$
\left(\mathcal{A}_{R}-\lambda\right)^{-1} f=\left(\mathcal{B}_{R}-\lambda g^{1 / 2}\right)^{-1}\left(g^{1 / 2} f\right) \text { for } f \in L_{g}^{2}(\Omega)
$$

where $\lambda \notin \operatorname{spec}\left(\mathcal{A}_{R}\right)$. We will use this formula extensively later for $f \in L^{2}(\Omega) \subset$ $L_{g}^{2}(\Omega)$. 
Lemma 2.10. The operator $\mathscr{B}_{R}-\lambda g^{1 / 2}$ has a bounded inverse if and only if $\lambda \notin$ $\operatorname{spec}\left(\mathcal{A}_{R}\right)$.

Proof. For $\lambda<0$ the operator $\mathscr{B}_{R}-\lambda g^{1 / 2}$ is strictly positive and, since $\mathscr{D}\left(\mathscr{B}_{R}\right) \subset$ $H_{0}^{1}(\Omega)$, has a compact resolvent. Therefore, the operator $\left(\mathcal{B}_{R}-\lambda g^{1 / 2}\right)^{-1}$ exists for $\lambda<0$ and is bounded in $L^{2}(\Omega)$. Since the multiplication, $u \mapsto g^{1 / 2} u$, is bounded in $L^{2}(\Omega)$, by the analytic Fredholm theory [64] the operator-valued function

$$
\lambda \mapsto\left(\mathscr{B}_{R}-\lambda g^{1 / 2}\right)^{-1}=\left[I-(\lambda+1)\left(\mathscr{B}_{R}+g^{1 / 2}\right)^{-1} g^{1 / 2}\right]^{-1}\left(\mathscr{B}_{R}+g^{1 / 2}\right)^{-1}
$$

is a meromorphic operator-valued function of $\lambda \in \mathbb{C}$. Therefore, if the inverse $\left(\mathscr{B}_{R}-\lambda g^{1 / 2}\right)^{-1}$ does not exist for a given $\lambda \geq 0$, then there is $v \in H_{0}^{1}(\Omega)$ such that

$$
\left(\mathscr{B}_{R}-\lambda g^{1 / 2}\right) v=0 .
$$

In this case $\mathscr{B}_{R} v=\lambda g^{1 / 2} v \in L^{2}\left(\Omega, g^{-1 / 2} d x\right)$ and we see that $v \in \mathscr{D}\left(\mathscr{A}_{R}\right)$, i.e. $\lambda \in \operatorname{spec}\left(\mathscr{A}_{R}\right)$. On the other hand, if $\lambda \in \operatorname{spec}\left(\mathscr{A}_{R}\right)$, i.e.

$$
-g^{-1 / 2} \nabla^{b} \cdot\left(\sigma_{R} \nabla^{b} u\right)+q u=\lambda u,
$$

then $\nabla^{b} \cdot\left(\sigma_{R} \nabla^{b} u\right)=q g^{1 / 2} u-\lambda g^{1 / 2} u \in L^{2}(\Omega)$, i.e. $u \in \mathscr{D}\left(\mathscr{B}_{R}\right)$, so that $\mathscr{B}_{R}-\lambda g^{1 / 2}$ does not have a bounded inverse.

Next we consider the uniform convergence of resolvents. To this end we introduce operators $\mathscr{B}_{R, m}, m \in \mathbb{Z}_{+}$, in $L^{2}(\Omega)$, of the form

$$
\begin{gathered}
\mathscr{B}_{R, m} u=-\nabla^{b} \cdot\left(\sigma_{R} \nabla^{b} u\right)+q g_{m}^{1 / 2} u, \\
\mathscr{D}\left(\mathscr{B}_{R, m}\right)=\left\{u \in H_{0}^{1}(\Omega): \nabla^{b} \cdot\left(\sigma_{R} \nabla^{b} u\right) \in L^{2}(\Omega)\right\}=\mathscr{D}\left(\mathscr{B}_{R}\right) .
\end{gathered}
$$

The operator $\mathcal{B}_{R, m}$ is associated with the operator $\mathcal{A}_{R, m}$ in the same way that $\mathcal{A}_{R}$ is associated with $\mathscr{B}_{R}$, where the operator $\mathcal{A}_{R, m}$ is the self-adjoint operator in $L^{2}\left(\Omega, g_{m}^{1 / 2} d x\right)$ defined by

$$
\begin{gathered}
\mathcal{A}_{R, m} u=-g_{m}^{-1 / 2} \nabla^{b} \cdot\left(\sigma_{R} \nabla^{b} u\right)+q u, \\
\mathscr{D}\left(\mathcal{A}_{R, m}\right)=\left\{u \in H_{0}^{1}(\Omega): \nabla^{b} \cdot\left(\sigma_{R} \nabla^{b} u\right) \in L^{2}(\Omega)\right\} .
\end{gathered}
$$

Note that $\mathscr{D}\left(\mathcal{A}_{R, m}\right)=\mathscr{D}\left(\mathscr{B}_{R, m}\right)$.

Lemma 2.11. Let $R>1$ and $K \subset \mathbb{C}$ be compact and such that $K \cap \operatorname{spec}\left(\mathcal{A}_{R}\right)=\emptyset$. Then there is an $m_{R} \in \mathbb{Z}_{+}$such that $K \cap \operatorname{spec}\left(\mathcal{A}_{R, m}\right)=\emptyset$ for $m>m_{R}$, and

$$
\lim _{m \rightarrow \infty}\left\|\left(\mathcal{A}_{R, m}-\lambda\right)^{-1}-\left(\mathcal{A}_{R}-\lambda\right)^{-1}\right\|_{L^{2}(\Omega) \rightarrow H_{0}^{1}(\Omega)}=0,
$$

uniformly for $\lambda \in K$. 
Proof. By the assumptions on $K$, the inverse $\left(\mathcal{B}_{R}-\lambda g^{1 / 2}\right)^{-1}$ exists and is a continuous function of $\lambda \in K$ with respect to the $L^{2}(\Omega)$-operator norm topology. Let

$$
d=\max _{\lambda \in K}\left\|\left(\mathscr{B}_{R}-\lambda g^{1 / 2}\right)^{-1}\right\|_{L^{2}(\Omega) \rightarrow L^{2}(\Omega)}<\infty .
$$

Denote $V_{R, m}(\lambda)=(\lambda-q)\left(g_{m}^{1 / 2}-g^{1 / 2}\right) \in L^{\infty}(\Omega)$, so that

$$
\lim _{m \rightarrow \infty}\left\|V_{R, m}(\lambda)\right\|_{L^{\infty}(\Omega)}=0
$$

uniformly for $\lambda \in K$. Thus there is $m(K)>0$ such that $\left\|V_{R, m}(\lambda)\right\|_{L^{\infty}} \leq(2 d)^{-1}$ for $m>m(K), \lambda \in K$. Therefore, $\left(\mathcal{B}_{R, m}-\lambda g_{m}^{1 / 2}\right)^{-1}$ exists for $\lambda \in K$ and is given by

$$
\left(\mathcal{B}_{R, m}-\lambda g_{m}^{1 / 2}\right)^{-1}=\left(\mathcal{B}_{R}-\lambda g^{1 / 2}\right)^{-1}\left[I+V_{R, m}(\lambda)\left(\mathcal{B}_{R}-\lambda g^{1 / 2}\right)^{-1}\right]^{-1}
$$

where the right hand side can be written as a Neumann series. This also shows that there is an $m_{R}$ such that $K \cap \operatorname{spec}\left(\mathcal{A}_{R, m}\right)=\emptyset$ for $m>m_{R}$.

For any $\lambda^{\prime} \in K,\left(\mathscr{B}_{R}-\lambda^{\prime} g^{1 / 2}\right)^{-1}$ is a bounded operator from $L^{2}(\Omega)$ to $H_{0}^{1}(\Omega)$, and if $|\lambda-\mu|<(2 d)^{-1}$ we have

$$
\left(\mathscr{B}_{R}-\lambda g^{1 / 2}\right)^{-1}=\left(\mathscr{B}_{R}-\lambda^{\prime} g^{1 / 2}\right)^{-1}\left[I+\left(\lambda^{\prime}-\lambda\right) g^{1 / 2}\left(\mathscr{B}_{R}-\lambda^{\prime} g^{1 / 2}\right)^{-1}\right]^{-1} .
$$

Using this we see that the norm of $\left(\mathscr{B}_{R}-\lambda g^{1 / 2}\right)^{-1}: L^{2}(\Omega) \rightarrow H_{0}^{1}(\Omega)$ is uniformly bounded in $\lambda \in K$. Using formulae (75) and (76), we see that

$$
\lim _{m \rightarrow \infty}\left\|\left(\mathscr{B}_{R, m}-\lambda g_{m}^{1 / 2}\right)^{-1}-\left(\mathscr{B}_{R}-\lambda g^{1 / 2}\right)^{-1}\right\|_{L^{2}(\Omega) \rightarrow H_{0}^{1}(\Omega)}=0
$$

uniformly for $\lambda \in K$, and that the norms of operators $\left(\mathscr{B}_{R, m}-\lambda g_{m}^{1 / 2}\right)^{-1}: L^{2}(\Omega) \rightarrow$ $H_{0}^{1}(\Omega)$ are uniformly bounded for $\lambda \in K$. This proves

$$
\lim _{m \rightarrow \infty}\left\|\left(\mathcal{B}_{R, m}-\lambda g_{m}^{1 / 2}\right)^{-1}-\left(\mathcal{B}_{R}-\lambda g^{1 / 2}\right)^{-1}\right\|_{L^{2}(\Omega) \rightarrow H_{0}^{1}(\Omega)}=0 .
$$

Additionally, the multiplication operators $g_{m}^{1 / 2}, g^{1 / 2}$ are bounded on $L^{2}(\Omega)$, uniformly in $m$, and

$$
\left\|g_{m}^{1 / 2}-g^{1 / 2}\right\|_{L^{2} \rightarrow L^{2}} \leq m^{-1 / 2} \rightarrow 0, \quad \text { as } m \rightarrow \infty .
$$

Together with eq. (77) and the boundedness of $\left(\mathcal{B}_{R, m}-\lambda g_{m}^{1 / 2}\right)^{-1}$ as operators from $L^{2}(\Omega)$ to $H_{0}^{1}(\Omega)$, this implies that

$$
\lim _{m \rightarrow \infty}\left\|\left(\mathcal{B}_{R, m}-\lambda g_{m}^{1 / 2}\right)^{-1} g_{m}^{1 / 2}-\left(\mathcal{B}_{R}-\lambda g^{1 / 2}\right)^{-1} g^{1 / 2}\right\|_{L^{2}(\Omega) \rightarrow H_{0}^{1}(\Omega)}=0 .
$$

This in turn implies eq. (74), due to formula (71) and the relations between $\mathscr{B}_{R, m}, \mathscr{B}_{R}$ and $\mathcal{A}_{R, m}, \mathcal{A}_{R}$, which follow from their definitions (30), (70)-(72), and (73). 
Let $R>1, \mu \notin \operatorname{spec}\left(\mathcal{A}_{R}\right)$. It follows from Lemma 2.11 that $\mu \notin \operatorname{spec}\left(\mathcal{A}_{R, m}\right)$ for $m$ sufficiently large. Denote by $N_{R, m}(\mu)$ the subspace of $L^{2}\left(\Omega, g_{m}^{1 / 2} d x\right)$ spanned by the eigenfunctions of $\mathcal{A}_{R . m}$ with eigenvalues in $(-\infty, \mu)$, cf. the definition of $N_{R}(\mu)$. Also denote by $P_{R, m}^{\mu}$ the orthogonal eigenprojectors onto $N_{R, m}(\mu)$ in $L^{2}\left(\Omega, g_{m}^{1 / 2} d x\right)$.

Clearly, since, for $m \in \mathbb{Z}_{+}, L^{2}\left(\Omega, g_{m}^{1 / 2} d x\right)=L^{2}(\Omega)$ as sets, we can consider $P_{R, m}^{\mu}$ as projectors, although not orthogonal, on $L^{2}(\Omega)$. Recall that $P_{R}^{\mu}$ is an orthoprojector in $L_{g}^{2}(\Omega)$ onto $N_{R}(\mu) \subset H_{0}^{1}(\Omega)$. Restricting it to $L^{2}(\Omega)$, we obtain a projector, which we still call $P_{R}^{\mu}$, on $L^{2}(\Omega)$. Again, $P_{R}^{\mu}$ is not an orthoprojector on $L^{2}(\Omega)$. However, we can compare these projectors, as well as spaces $N_{R, m}(\mu)$ and $N_{R}(\mu)$.

Corollary 2.12. Let $R>1$ and $\mu \notin \operatorname{spec}\left(\mathcal{A}_{R}\right)$. The Riesz projectors $P_{R, m}^{\mu}$ corresponding to the operators $\mathcal{A}_{R, m}$ satisfy

$$
\lim _{m \rightarrow \infty}\left\|P_{R, m}^{\mu}-P_{R}^{\mu}\right\|_{L^{2}(\Omega) \rightarrow H_{0}^{1}(\Omega)}=0 .
$$

Moreover, for $m$ sufficiently large,

$$
\operatorname{dim}\left(N_{R, m}(\mu)\right)=\operatorname{dim}\left(N_{R}(\mu)\right) .
$$

Proof. Let $\Gamma \subset \mathbb{C}$ be a contour surrounding only $\lambda_{0}$ from $\operatorname{spec}\left(\mathcal{A}_{R}\right)$. When $m$ is large enough, the Riesz projectors $P_{R, m}^{\mu}$ have a representation analogous to (62), obtained by replacing $\mathcal{A}_{R}$ by $\mathcal{A}_{R, m}$ and using the contour $\Gamma$. Thus

$$
\begin{aligned}
& \lim _{m \rightarrow \infty}\left\|P_{R, m}^{\mu}-P_{R}^{\mu}\right\|_{L^{2}(\Omega) \rightarrow H_{0}^{1}(\Omega)} \\
& \quad \leq \lim _{m \rightarrow \infty} \frac{1}{2 \pi} \int_{\Gamma}\left\|\left(\mathcal{A}_{R, m}-z\right)^{-1}-\left(\mathcal{A}_{R}-z\right)^{-1}\right\|_{L^{2}(\Omega) \rightarrow H_{0}^{1}(\Omega)} d l(z),
\end{aligned}
$$

where $d l$ is the arclength measure on $\Gamma$. Taking into account (74), this formula implies (78).

Using eq. (78), we see that there exists an $m_{0}$ such that for $m \geq m_{0}$ we have $\left\|P_{R, m}^{\mu}-P_{R}^{\mu}\right\|_{L^{2}(\Omega) \rightarrow L^{2}(\Omega)}<1$. Using, e.g., Corollary IV.2.6 in [39], we see that this proves (79).

\section{Approximating anisotropic by isotropic mass densities}

We now show, using techniques from homogenization theory, cf. [1], [15], and [18], that we can approximate arbitrarily closely, on the level of the operators, the nonsingular anisotropic approximate mass densities $\sigma_{R}^{-1}$, for any fixed $R>1$, by a family of nonsingular isotropic mass densities $\sigma_{R, \varepsilon}^{-1}, \varepsilon>0$, which will thus also function as approximate cloaks. This can be considered as the reverse of the traditional homogenization theory. 
3.1. Inverse homogenization with magnetic potential. Observe that, as all the approximate cloaks $\sigma_{R}, R>1$, are rotationally invariant, it is natural to use spherical coordinates. Namely, we will use either the Euclidian coordinates $x=\left(x^{1}, x^{2}, x^{3}\right)$, or the spherical coordinates $s=s(x)=(r(x), \theta(x), \varphi(x))$. Note that we use the same notation $x$ for a point inside $\Omega$ and its Euclidian coordinates, $x=\left(x^{1}, x^{2}, x^{3}\right)$. Which meaning is intended will be always clear from the context. We denote by $X:\left(x^{1}, x^{2}, x^{3}\right) \mapsto(r, \theta, \varphi)$ the corresponding coordinate transformation. To exploit the rotational invariance, we will employ in the homogenization process, cf. [1] and [15], cells which are cubes in these spherical coordinates.

To approximate the anisotropic mass densities in spherical coordinates in the ball $\Omega=B(0,3)$, let us consider isotropic mass densities which inverse is of the form

$$
\sigma_{\varepsilon}(x)=\sigma(x, r(x) / \varepsilon), \quad \sigma\left(x, r^{\prime}\right)=h\left(x, r^{\prime}\right) I \in \mathbb{R}^{3 \times 3} .
$$

Here $h\left(x, r^{\prime}\right)$ is a scalar valued function, to be chosen later, that is periodic in $r^{\prime}$ with period 1 and is bounded from above and below, i.e.

$$
h\left(x, r^{\prime}+1\right)=h\left(x, r^{\prime}\right), \quad 0<c_{1} \leq h\left(x, r^{\prime}\right) \leq c_{2} .
$$

We will consider densities for which $h\left(x, r^{\prime}\right)$ is independent of $r^{\prime}$ for $x$ with $r(x)<1$ and $5 / 2<r(x)<3$, that is,

$$
h\left(x, r^{\prime}\right)=h(x) \text { if }|x|<1 \text { or } 5 / 2<|x|<3 .
$$

We make this assumption since later we will use the isotropic mass densities to approximate the non-singular anisotropic mass densities $\sigma_{R}^{-1}, R>1$ that are isotropic for $r(x)<1$ and $5 / 2<r(x)<3$.

Let $(r, \theta, \varphi)$ and $\left(r^{\prime}, \theta^{\prime}, \varphi^{\prime}\right)$ be spherical coordinates corresponding to two different scales. Then, in these coordinates,

$$
\left(X_{*}\left(\sigma_{\varepsilon}\right)\right)(s)=\sigma^{*}(s, s / \varepsilon),
$$

where

$$
\begin{aligned}
\sigma^{*}\left(s, r^{\prime}\right) & =\left.h\left(s, r^{\prime}\right) \operatorname{det}(D X(x))^{-1} D X(x) D X^{t}(x)\right|_{X(x)=s} \\
& =h\left(s, r^{\prime}\right)\left(\begin{array}{ccc}
r^{2} \sin \theta & 0 & 0 \\
0 & \sin \theta & 0 \\
0 & 0 & 1 / \sin \theta
\end{array}\right) .
\end{aligned}
$$

Here and later we denote by $\sigma$, sometimes with various indices, the inverse of the various mass tensors (or matrices) in the Euclidian coordinates, while $\sigma^{*}$ always stands for their representation in the spherical coordinates.

In the following, the material on homogenization is a quite straightforward generalization of known results [1] and [15]. However, as we need to introduce changes due both to the presence of a magnetic potential and the use of spherical coordinates, for completeness we give details of the arguments. 
In the small-scale coordinates $t=\left(r^{\prime}, \varphi^{\prime}, \theta^{\prime}\right)$, we denote by $e^{1}=(1,0,0)$, $e^{2}=(0,1,0)$, and $e^{3}=(0,0,1)$ the vectors corresponding to the differential forms $d r^{\prime}, d \theta^{\prime}$, and $d \varphi^{\prime}$, respectively. Let $W^{j}(s, t), j=1,2,3$, be the solutions of

$$
\nabla_{t} \cdot \sigma^{*}(s, t)\left(\nabla_{t} W^{j}(s, t)+e^{j}\right)=0, \quad t=\left(r^{\prime}, \theta^{\prime}, \varphi^{\prime}\right) \in \mathbb{R}^{3},
$$

that are 1-periodic functions in $r^{\prime}, \theta^{\prime}$, and $\varphi^{\prime}$ variables (noting that the periodicity in $r^{\prime}$, $\theta^{\prime}$, and $\varphi^{\prime}$ has no relation to periodicity in the "large-scale" spherical coordinates $\theta$, $\varphi$ ), and satisfy $\int_{[0,1]^{3}} W^{j}(s, t) d t=0$ for all $s$, where $d t=d r^{\prime} d \theta^{\prime} d \varphi^{\prime}$. Since $\sigma^{*}$ is independent of $\theta^{\prime}, \varphi^{\prime}$, the above conditions imply that $W^{j}=0$ for $j=2,3$. As for $W^{1}$, it satisfies

$$
\frac{\partial}{\partial r^{\prime}}\left(h\left(s, r^{\prime}\right) \frac{\partial W^{1}}{\partial r^{\prime}}\right)=-\frac{\partial h\left(s, r^{\prime}\right)}{\partial r^{\prime}},
$$

with $W^{1}$ being 1-periodic with respect to $\left(\theta^{\prime}, \varphi^{\prime}\right)$. These imply that $W^{1}$ is independent of $\left(\theta^{\prime}, \varphi^{\prime}\right)$ with

$$
\frac{\partial W^{1}}{\partial r^{\prime}}=-1+\frac{C_{0}}{h\left(s, r^{\prime}\right)} .
$$

To find the constant $C_{0}$ we use the periodicity of $W^{1}$, now with respect to $r^{\prime}$, to get that $C_{0}$ is given by the harmonic means $h^{\text {harm }}$ of $h$,

$$
C_{0}=h^{\text {harm }}(s)=\left(\int_{0}^{1} \frac{d r^{\prime}}{h\left(s, r^{\prime}\right)}\right)^{-1}
$$

Define the corrector matrices [1] as

$$
P_{j}^{k}(s, t)=\frac{\partial}{\partial t^{j}} W^{k}(s, t)+\delta_{j}^{k} .
$$

Then the inverse of the homogenized mass density in the spherical coordinates, $\sigma_{\mathrm{hom}}^{*}$, is given by

$$
\left(\sigma_{\text {hom }}^{*}\right)^{j k}(s)=\sum_{p=1}^{3} \int_{[0,1]^{3}}\left(\sigma^{*}\right)^{j p}(s, t) P_{p}^{k}(s, t) d t .
$$

We note that, applying integration by parts and using definition (85) and eq. (83), eq. (86) can be written also in a more symmetric form

$$
\left(\sigma_{\mathrm{hom}}^{*}\right)^{j k}(s)=\sum_{p, q=1}^{3} \int_{[0,1]^{3}}\left(\sigma^{*}\right)^{p q}(s, t) P_{p}^{j}(s, t) P_{q}^{k}(s, t) d t .
$$

Using the above formulae for the $W^{i}$, it follows from (86) that

$$
\sigma_{\mathrm{hom}}^{*}(s)=\left(\begin{array}{ccc}
h^{\mathrm{harm}}(s) r^{2} \sin (\theta) & 0 & 0 \\
0 & h^{a}(s) \sin (\theta) & 0 \\
0 & 0 & h^{a}(s) \sin ^{-1}(\theta)
\end{array}\right) .
$$


Here $h^{a}(s)$ denotes the arithmetic means of $h$ in the second variable,

$$
h^{a}(s)=\int_{[0,1]} h\left(s, r^{\prime}\right) d r^{\prime}
$$

Returning to the Euclidian coordinates, one sees that the conductivity, $\sigma_{\text {hom }}(x)=$ $X_{*} \sigma_{\text {hom }}^{*}(x)$, has the form

$$
\sigma_{\text {hom }}(x)=\omega_{1}(x) \Pi(x)+\omega_{2}(x)(I-\Pi(x)),
$$

with

$$
\omega_{1}(x)=h^{\text {harm }}(x), \quad \omega_{2}(x)=h^{a}(x)
$$

and $\Pi(x): \mathbb{R}^{3} \rightarrow \mathbb{R}^{3}$ being the projection to the radial direction,

$$
\Pi(x) v=\left(v \cdot \frac{x}{|x|}\right) \frac{x}{|x|},
$$

represented by the matrix $\left(|x|^{-2} x^{j} x^{k}\right)_{j, k=1}^{3}$.

Next, we analyze the Dirichlet problems for elliptic equations

$$
-\nabla^{b} \cdot \sigma_{\varepsilon} \nabla^{b} u_{\varepsilon}+Q u_{\varepsilon}=f,\left.\quad u_{\varepsilon}\right|_{\partial \Omega}=h
$$

Here, $b(x)=\left(b_{1}(x), b_{2}(x), b_{3}(x)\right)$ is the magnetic potential and $Q(x)$ is a scalar function, with $Q, b_{j} \in L^{\infty}(\Omega ; \mathbb{R})$, and $\sigma_{\varepsilon}^{-1}$ are isotropic mass densities bounded from above and below by positive constants independent of $\varepsilon$. Moreover, $f \in H^{-1}(\Omega)$, and $h \in H^{1 / 2}(\partial \Omega)$. Our goal is to show that the solutions $u_{\varepsilon}$ convergence to the solution of the equation

$$
-\nabla^{b} \cdot \sigma_{\mathrm{hom}} \nabla^{b} u+Q u=f,\left.\quad u\right|_{\partial \Omega}=h .
$$

By adapting the technique of Allaire [1], we can prove the following result.

Proposition 3.1. Let $\sigma_{\varepsilon}, \varepsilon>0$ be the inverse matrices of the mass densities in $\Omega$ satisfying (80), (81), and (82), $\sigma_{\mathrm{hom}}$ be the inverse of the mass density defined by (87), $Q \in L^{\infty}(\Omega), Q(x) \geq 0$, and $b=\left(b_{1}(x), b_{2}(x), b_{3}(x)\right)$ be a vector field, $b \in L^{\infty}\left(\Omega ; \mathbb{R}^{3}\right)$. Then the solutions $u_{\varepsilon}$ of (89) and solution $u$ of (90) satisfy

$$
\lim _{\varepsilon \rightarrow 0} u_{\varepsilon}=u \text { weakly in } H^{1}(\Omega) \text {. }
$$

Proof. Let $E_{h} \in H^{1}(\Omega), \operatorname{supp}\left(E_{h}\right) \subset\{5 / 2 \leq|x| \leq 3\}$ be an extension of $h$, i.e. $E_{\left.h\right|_{\partial \Omega}}=h$. Writing $u_{\varepsilon}=E_{h}+v_{\varepsilon}$ and $u=E_{h}+v$, we see that the functions $v_{\varepsilon}$ and $v$ satisfy

$$
-\nabla^{b} \cdot \sigma_{\varepsilon} \nabla^{b} v_{\varepsilon}+Q v_{\varepsilon}=\tilde{f} \quad \text { on } \Omega,\left.\quad v_{\varepsilon}\right|_{\partial \Omega}=0
$$




$$
-\nabla^{b} \cdot \sigma_{\mathrm{hom}} \nabla^{b} v+Q v=\tilde{f} \text { on } \Omega,\left.\quad v\right|_{\partial \Omega}=0 .
$$

Here

$$
\tilde{f}=f-\nabla^{b} \cdot \sigma_{\varepsilon} \nabla^{b} E_{h}+Q E_{h} \in H^{-1}(\Omega),
$$

is independent of $\varepsilon$. For proving (91) it is enough to show that $v_{\varepsilon}$ converges to $v$ weakly in $H_{0}^{1}(\Omega)$.

Let us recall that by, Lemma 2.2, $a_{R}$ is (strictly) positive definite. Since $Q(x) \geq 0$, we see that there exists a $c_{0}>0$ such that

$$
\int_{\Omega}\left(\left|\nabla^{b} u(x)\right|^{2}+Q(x)|u(x)|^{2}\right) d x \geq c_{0}\|u\|_{H_{0}^{1}(\Omega)}^{2} \text { for } u \in H_{0}^{1}(\Omega) .
$$

As the mass densities $\sigma_{\varepsilon}^{-1}$ are uniformly bounded from above and below by positive constants, it follows from the proof of Lemma 2.2 that there is also $c_{1}>0$ such that

$$
\int_{\Omega}\left(\nabla^{b} u \cdot \sigma_{\varepsilon} \overline{\nabla^{b} u}+Q(x)|u|^{2}\right) d x \geq c_{1}\|u\|_{H_{0}^{1}(\Omega)}^{2} \text { for } u \in H_{0}^{1}(\Omega) .
$$

Thus, using the Lax-Milgram lemma, we see that the solutions $v_{\varepsilon}(x)$ of (92) satisfy

$$
\left\|v_{\varepsilon}\right\|_{H_{0}^{1}(\Omega)} \leq c\|\tilde{f}\|_{H^{-1}(\Omega)},
$$

where $c>0$ is independent of $\varepsilon>0$. Therefore, the solutions $v_{\varepsilon}(x)$ are uniformly bounded in $H_{0}^{1}(\Omega)$. Thus, for an arbitrary sequence $\varepsilon_{n} \rightarrow 0$, the corresponding $v_{\varepsilon_{n}}$ have a subsequence that converges weakly to some function $w(x)$ in $H_{0}^{1}(\Omega)$. Let us show that $w$ coincides with the solution $v$ of (93).

To this end we consider convergence in a finer, two-scale, sense on local coordinate neighborhoods. Let $U \subset \mathbb{S}^{2}$ be an open set on which we can define, in a regular manner, spherical coordinates. For example, by choosing two antipodal points as the South and North poles and connecting those by a meridian $\gamma$, we can take $U$ so that $\bar{U} \subset \mathbb{S}^{2} \backslash \gamma$ and define polar coordinates on $U$. Let $\Omega^{\prime}=\{r \omega: \omega \in U, r \in$ $\left.\left(r_{1}, r_{2}\right)\right\} \subset \Omega$ with some $r_{2}>r_{1}>0$. Clearly, $X: \Omega^{\prime} \rightarrow \mathbb{R}^{3}$ defines the spherical coordinates, $x \mapsto(r, \theta, \varphi)$, with domain $W=X\left(\Omega^{\prime}\right)=\left(r_{1}, r_{2}\right) \times U$.

Rewrite now eq. (92) and (93) on $W$ in these spherical coordinates and multiply the equations so obtained by $\operatorname{det}\left(D X\left(X^{-1}(s)\right)\right)$. The resulting equations are

$$
\begin{gathered}
-\left(\nabla_{s}+i b^{*}(s)\right) \cdot \sigma^{*}\left(s, \frac{s}{\varepsilon}\right)\left(\nabla_{s}+i b^{*}(s)\right) v_{\varepsilon}(s)+Q^{*}(s) v_{\varepsilon}(s)=\hat{f}(s), \\
-\left(\nabla_{s}+i b^{*}(s)\right) \cdot \sigma_{\mathrm{hom}}^{*}(s)\left(\nabla_{s}+i b^{*}(s)\right) v(s)+Q^{*}(s) v(s)=\hat{f}(s),
\end{gathered}
$$

where $s \in W$. Here

$$
\begin{aligned}
Q^{*}(s) & =\operatorname{det}\left(D X\left(X^{-1}(s)\right)\right) Q\left(X^{-1}(s)\right) \in L^{\infty}(W), \\
b_{j}^{*}(s) & =\partial_{j} X^{k}\left(X^{-1}(s)\right) b_{k}\left(X^{-1}(s)\right) \in L^{\infty}\left(W ; \mathbb{R}^{3}\right),
\end{aligned}
$$


correspond to the electric potential $Q$ and magnetic potential $b$ in the spherical coordinates and $\hat{f}(s)=\operatorname{det}\left(D X\left(X^{-1}(s)\right)\right) \tilde{f}\left(X^{-1}(s)\right) \in H^{-1}(W)$. To simplify notations, we continue to denote the functions $v$ and $v_{\varepsilon}$ in the spherical coordinates by $v(s)=\left(\left(X^{-1}\right)^{*} v\right)(s), v_{\varepsilon}(s)=\left(\left(X^{-1}\right)^{*} v_{\varepsilon}\right)(s)$. Finally, $\nabla_{s}$ is the vector field $\left(\partial_{r}, \partial_{\theta}, \partial_{\varphi}\right)$.

In the following, even though in the forthcoming applications the mass densities $\left(\sigma_{\varepsilon}^{*}(x, r(x) / \varepsilon)\right)^{-1}$ will depend only on the small-scale variable $r^{\prime}=r(x) / \varepsilon$, we consider the general case when the mass densities depend on all small-scale coordinates $t=\left(r^{\prime}, \theta^{\prime}, \varphi^{\prime}\right)$. Let $T=[0,1]^{3}$ and $C_{\#}^{m}(T)$ denote those $C^{m}(T)$ functions that can be continued as $\mathbb{Z}^{3}$-periodic functions in $\mathbb{R}^{3}$ which are in $C^{m}\left(\mathbb{R}^{3}\right)$. By definition, a family $v_{\varepsilon}(s), v_{\varepsilon} \in L^{2}(W)$ is said to two-scale converge, as $\varepsilon \rightarrow 0$, to a function $v_{0}(s, t) \in L^{2}(W \times T)$ if, for all test functions $\psi(s, t)$ in $C_{0}^{\infty}\left(W ; C_{\#}^{\infty}(T)\right)$, we have

$$
\lim _{\varepsilon \rightarrow 0} \int_{W} v_{\varepsilon}(s) \psi\left(s, \frac{s}{\varepsilon}\right) d s=\int_{W} \int_{T} v_{0}(s, t) \psi(s, t) d s d t .
$$

By [1], the two-scale convergence of $v_{\varepsilon}$ implies the weak convergence of $v_{\varepsilon}(s)$ in $L^{2}(W)$ to the function $w(s)=\int_{T} v_{0}(s, t) d t$, so that the two-scale convergence gives finer information on the convergence than the weak convergence. For example, functions of the form $u(s, s / \varepsilon)$ two-scale converge to $u(s, t)$.

By [1], every bounded family $v_{\varepsilon}(s) \in L^{2}(W)$ contains a two-scale converging sequence. Moreover, if $v_{\varepsilon}(s)$ is a bounded family in $H^{1}(W)$ that converges in $L^{2}(W)$ to $w(s)$ as $\varepsilon \rightarrow 0$, then $v_{\varepsilon}(s)$ also two-scale converges to $w(s)$ and there is a function $w_{1}(s, t) \in L^{2}(W \times T)$, so that $\nabla_{s} v_{\varepsilon}(s)$ two-scale converge to $\nabla_{s} w(s)+\nabla_{t} w_{1}(s, t)$. For example, if functions $v_{\varepsilon}(s)$ have the form $v_{\varepsilon}(s)=u_{0}(s)+\varepsilon u_{1}(s, s / \varepsilon)$, then $\nabla_{s} v_{\varepsilon}(s)$ two-scale converge to $u(s, t)=\nabla u_{0}(s)+\nabla_{t} u_{1}(s, t)$.

As noted above, the solutions $v_{\varepsilon}(x)$ of eq. (92) are uniformly bounded in $H_{0}^{1}(\Omega)$. Consider the restrictions of these functions on $\Omega^{\prime}$ and rewrite them in the spherical coordinates on $W$. Then any sequence of $v_{\varepsilon}(s)$ has a subsequence $v_{\varepsilon_{j}}(s)$, $j \in \mathbb{Z}_{+}, \varepsilon_{j} \rightarrow 0$ as $j \rightarrow \infty$, that weakly converges, in $H^{1}(W)$, to some function $w(s)$. By [1], $v_{\varepsilon_{j}}(s)$ also two-scale converge to $w(s)$, as $\varepsilon \rightarrow 0$, and there is a function $w_{1}(s, t) \in L^{2}(W \times T)$ so that $\nabla v_{\varepsilon_{j}}(s)$ two-scale converge to $\nabla_{s} w(s)+\nabla_{t} w_{1}(s, t)$.

Let us now multiply both sides of eq. (96) by a test function $\varphi(s)+\varepsilon \varphi_{1}(s, s / \varepsilon)$, where $\varphi(s) \in C_{0}^{\infty}(W)$ and $\varphi_{1}(s, t) \in C_{0}^{\infty}\left(W ; C_{\#}^{\infty}(T)\right)$, and integrate over $W$. Using integration by parts, we obtain

$$
\begin{gathered}
\int_{W} \sigma^{*}\left(s, \frac{s}{\varepsilon}\right)\left[\left(\nabla_{s}+i b^{*}(s)\right) v_{\varepsilon}(s)\right] \cdot \overline{\left(\eta_{1}\left(s, \frac{s}{\varepsilon}\right)+\varepsilon \eta_{2}\left(s, \frac{s}{\varepsilon}\right)\right)} d s \\
+\int_{W} Q^{*}(s) v_{\varepsilon}(s) \overline{\left[\varphi(s)+\varepsilon \varphi_{1}\left(s, \frac{s}{\varepsilon}\right)\right]} d s \\
=\int_{\Omega^{\prime}} \hat{f}(s) \overline{\left[\varphi(s)+\varepsilon \varphi_{1}\left(s, \frac{s}{\varepsilon}\right)\right]} d s,
\end{gathered}
$$


where

$$
\begin{aligned}
& \eta_{1}(s, t)=\nabla_{s} \varphi(s)+i b^{*}(s) \varphi(s)+\nabla_{t} \varphi_{1}(s, t), \\
& \eta_{2}(s, t)=\nabla_{s} \varphi_{1}(s, t)+i b^{*}(s) \varphi_{1}(s, t) .
\end{aligned}
$$

Substitute $\varepsilon=\varepsilon_{j}$ in (99) and let $j \rightarrow \infty$. First, we observe that, since both $\eta_{2}(s, s / \varepsilon)$ and $\varepsilon \in(0,1)$ are uniformly bounded in $L^{2}(W)$, the terms in (99), involving $\eta_{2}$, tend to zero. Second, as $v_{\varepsilon}(s)$ converge in strong topology of $L^{2}(W)$ to $w$, the first integral in (99) tends to $\int_{W} Q^{*}(s) w(s) \overline{\varphi(s)} d s$. Third, the inner product of $\hat{f}(s)$ and $\varepsilon \varphi_{1}$ in the last integral in (99) goes to zero. Fourth, the functions $\psi_{\varepsilon}(s)=$ $\sigma^{*}(s, s / \varepsilon) \eta_{1}(s, s / \varepsilon)$ two-scale converge to the function $\psi(s, t)=\sigma^{*}(s, t) \eta_{1}(s, t)$. Since $\psi(x, y) \in L^{2}\left(\Omega ; C_{\#}^{0}(T)\right)$, we have by Lemma 1.3 in [1] that

$$
\lim _{\varepsilon \rightarrow 0}\left\|\psi_{\varepsilon}\right\|_{L^{2}(W)}=\|\psi\|_{L^{2}(W \times T)} .
$$

Furthermore, as $\nabla v_{\varepsilon_{j}}(s)$ two-scale converges to $\nabla_{s} w(s)+\nabla_{t} w_{1}(s, t)$ it follows from (100) and Theorem 1.8 in [1], that

$$
\begin{aligned}
\int_{W} \sigma^{*}\left(s, \frac{s}{\varepsilon j}\right)\left[\left(\nabla_{s}+i b^{*}(s)\right) v_{\varepsilon_{j}}(s)\right] \cdot \overline{\eta_{1}\left(s, \frac{s}{\varepsilon}\right)} d s \\
\rightarrow \int_{W} \int_{T} \sigma^{*}(s, t)\left[\nabla_{s} w(s)+\nabla_{t} w_{1}(s, t)\right] \cdot \overline{\eta_{1}(s, t)} d s d t,
\end{aligned}
$$

as $j \rightarrow \infty$. Summarizing, we see that

$$
\begin{aligned}
& \int_{W} \int_{T} \sigma^{*}(s, t)\left[\nabla_{s} w(s)+\nabla_{t} w_{1}(s, t)+i b^{*}(s) w(s)\right] \\
& \quad+\int_{W} \int_{T} Q^{*}(s) w(s) \overline{\varphi\left(\nabla_{s} \varphi(s)+i b^{*}(s) \varphi(x)+\left(\nabla_{t} \varphi_{1}\right)(s, t)\right]} d s d t \\
& \quad=\int_{W} \hat{f}(s) \overline{\varphi(s)} d s .
\end{aligned}
$$

Taking $\varphi(s)=0$ in (101) and varying $\varphi_{1}(s, t)$ over all test functions, integration by parts with respect to $t$ shows that

$$
\nabla_{t} \cdot \sigma^{*}(s, t)\left[\nabla_{s} w(s)+\nabla_{t} w_{1}(s, t)+i b^{*}(s) w(s)\right]=0 \text { a.e. in } W \times T .
$$

Since $\nabla_{s} w(s)+i b^{*}(s) w(s)$ depends only on $s$ and thus can be considered as a constant vector in the $t$ variable, we see that

$$
w_{1}(s, t)=\sum_{j=1}^{3}\left(\frac{\partial w}{\partial s^{j}}(s)+i b_{j}^{*}(s) w(s)\right) W^{j}(s, t) \text { a.e. in } W \times T,
$$


where $W^{j}$ are solutions of the cell eq. (83).

On the other hand, taking $\varphi_{1}(s, t)=0$ in (101) and varying $\varphi(s)$ over all test functions, we see, using integration by parts with respect to $s$, that

$$
\begin{aligned}
-\left(\nabla_{s}+\right. & \left.i b^{*}(s)\right) \cdot\left(\int_{T} \sigma^{*}(s, t)\left[\nabla_{s} w(s)+\nabla_{t} w_{1}(s, t)+i b^{*}(s) w(s)\right] d t\right) \\
& +Q^{*}(s) w(s) \\
= & \hat{f}(s) \quad \text { a.e. in } W .
\end{aligned}
$$

Comparing the above integral with eq. (86) for $\sigma_{\text {hom }}$, we see that (103) and (104) imply that

$$
-\left(\nabla_{s}+i b^{*}(s)\right) \cdot \sigma_{\mathrm{hom}}^{*}(s)\left(\nabla_{s}+i b^{*}(s)\right) w(s)+Q^{*}(s) w(s)=\hat{f}(s) \text { in } W,
$$

or equivalently, in the Cartesian coordinates

$$
-(\nabla+i b) \cdot \sigma_{\mathrm{hom}}(\nabla+i b) w+Q w=\tilde{f} \text { in } \Omega^{\prime} .
$$

Since $\Omega \backslash\{O\}$ can be covered with coordinate neighborhoods $\Omega^{\prime}$ used above, eq. (105) is valid in the domain $\Omega \backslash\{O\}$. Moreover, since all the inverse matrices of the mass densities $\sigma_{\text {hom }}$ and $\sigma_{\varepsilon}$ are the same near the origin, we see that all functions $v_{\varepsilon_{j}}(x)$ satisfy eq. (105) near the origin. As $v_{\varepsilon_{j}}(x)$ converge weakly in $H_{0}^{1}(\Omega)$ to $w(x)$, eq. (105) is valid in $\Omega$. This means that $w(x)$, as well as $v(x)$, is the solution of the eq. (93). By the Lax-Milgram Theorem, eq. (93) has a unique solution, and thus $w=v$.

Summarizing, we have now shown that an arbitrary sequence of the original family $v_{\varepsilon}(x)$ has a subsequence that weakly converges, in $H_{0}^{1}(\Omega)$, to the solution $v(x)$ of eq. (93). This shows that the whole family $v_{\varepsilon}(x)$ of the solutions of the eq. (92) converge weakly to $v(x)$ in $H_{0}^{1}(\Omega)$.

3.2. Approximation of $\sigma_{R}$. Next we apply the above results to approximate the inverse matrices of the mass densities $\sigma_{R}$ for a fixed $R>1$. In the forthcoming analysis, we will choose the function $h$ to be

$$
h\left(s, r^{\prime}\right)=\frac{a(s)}{1+b(s) p\left(r^{\prime}\right)},
$$

where $p\left(r^{\prime}\right)$ is a fixed positive, smooth 1-periodic function of $r^{\prime}$ and both $a(s)$ and $b(s)$ are positive. For any $x \in \Omega$ and a given function $p\left(r^{\prime}\right)$, the pair $\left(\omega_{1}(x), \omega_{2}(x)\right)$ in (87) and (88) depends only on the values of functions $(a(s(x)), b(s(x)))$. By choosing $(a(s), b(s))$ appropriately, it is possible to achieve any value

$$
\left(\omega_{1}(x), \omega_{2}(x)\right) \in \mathbb{R}_{+}^{2} \text {, with } \omega_{1}(x) \leq \omega_{2}(x) .
$$


We can choose $a(x)=a_{R}(x)$ and $b(x)=b_{R}(x)$ so that $\sigma_{\text {hom }}(x)$ corresponds to the inverse of the approximate cloaking mass density, that is

$$
\sigma_{\text {hom }}(x)=\sigma_{R}(x), \quad x \in \Omega .
$$

In the sequel, we will denote by $\sigma_{R}\left(x, r^{\prime}\right)^{-1}=h_{R}\left(x, r^{\prime}\right)^{-1} I$ the isotropic mass densities for which the homogenized mass densities $\sigma_{\text {hom }}^{-1}$ satisfy (107). Observe that, for $R>1$, we can choose $h_{R}\left(s, r^{\prime}\right)$ so that

$$
0<c_{1}(R) \leq h_{R}\left(s, r^{\prime}\right) \leq c_{2}, \quad c_{1}(R) \rightarrow 0 \text { as } R \searrow 1 .
$$

Let $\lambda<0$ and $\mathscr{B}_{R, m, \varepsilon}$ be the operators

$$
\begin{gathered}
\mathcal{B}_{R, m, \varepsilon} u=-\nabla^{b} \cdot \sigma_{R, \varepsilon} \nabla^{b} u+g_{m}^{1 / 2} q u, \\
\mathscr{D}\left(\mathcal{B}_{R, m, \varepsilon}\right)=\left\{u \in H_{0}^{1}(\Omega):-\nabla^{b} \cdot \sigma_{R, \varepsilon} \nabla^{b} u \in L^{2}(\Omega)\right\} .
\end{gathered}
$$

Lemma 3.2. For any $\lambda<0$ and $f \in L^{2}(\Omega)$,

$$
\lim _{\varepsilon \rightarrow 0}\left(\mathscr{B}_{R, m, \varepsilon}-\lambda g_{m}^{1 / 2}\right)^{-1} f=\left(\mathscr{B}_{R, m}-\lambda g_{m}^{1 / 2}\right)^{-1} f,
$$

where $\mathscr{B}_{R, m}$ is as in (72), and the limit holds both in the sense of the strong topology in $L^{2}(\Omega)$ and weak topology in $H^{1}(\Omega)$.

Proof. This follows immediately from Proposition 3.1 by taking $Q=(q-\lambda) g_{m}^{1 / 2}$.

As in the previous section, one can analyze the convergence of the resolvents in more detail. To this end, introduce operators $\mathcal{A}_{R, m, \varepsilon}=g_{m}^{-1 / 2} \mathscr{B}_{R, m, \varepsilon}$ that is,

$$
\begin{gathered}
\mathcal{A}_{R, m, e}=-g_{m}^{-1 / 2} \nabla^{b} \cdot \sigma_{R, \varepsilon} \nabla^{b} u+q u, \\
\mathscr{D}\left(\mathcal{A}_{R, m, \varepsilon}\right)=\mathscr{D}\left(\mathcal{B}_{R, m, \varepsilon}\right)=\left\{u \in H_{0}^{1}(\Omega):-\nabla^{b} \cdot \sigma_{R, \varepsilon} \nabla^{b} u \in L^{2}(\Omega)\right\} .
\end{gathered}
$$

Clearly, the operators $\mathcal{A}_{R, m, e}$ are self-adjoint in $L^{2}\left(\Omega, g_{m}^{1 / 2} d x\right)$ and

$$
\left(\mathcal{A}_{R, m, \varepsilon}-\lambda\right)^{-1} f=\left(\mathscr{B}_{R, m, \varepsilon}-\lambda g_{m}^{1 / 2}\right)^{-1}\left(g_{m}^{1 / 2} f\right)
$$

for $\lambda \notin \operatorname{spec}\left(\mathcal{A}_{R, m, \varepsilon}\right)$ and $f \in L^{2}(\Omega)$, cf. eq. (72) and (73).

Lemma 3.3. Let $R>1, m \in \mathbb{Z}_{+}$and $K \subset \mathbb{C}$ be compact and such that

$$
\operatorname{dist}\left(K, \operatorname{spec}\left(\mathcal{A}_{R, m}\right)\right) \geq \delta_{0}>0 .
$$

(i) There is an $\varepsilon_{0}=\varepsilon_{0}(K, R, m)$ such that such that, for $0<\varepsilon<\varepsilon_{0}$,

$$
\left\|\left(\mathcal{A}_{R, m, \varepsilon}-\lambda\right)^{-1}\right\|_{L^{2}\left(\Omega, g_{m}^{1 / 2} d x\right) \rightarrow L^{2}\left(\Omega, g_{m}^{1 / 2} d x\right)} \leq \frac{2}{\delta_{0}}, \quad \lambda \in K .
$$


(ii) For any $f \in L^{2}(\Omega)$,

$$
\lim _{\varepsilon \rightarrow 0}\left(\mathcal{A}_{R, m, \varepsilon}-\lambda\right)^{-1} f=\left(\mathcal{A}_{R, m}-\lambda\right)^{-1} f
$$

in the strong topology of $L^{2}(\Omega)$ and weak topology of $H_{0}^{1}(\Omega)$, with convergence being uniform for $\lambda \in K$.

Proof. (i) Since the $\mathcal{A}_{R, m, \varepsilon}$ are self-adjoint operators on $L^{2}\left(\Omega, g_{m}^{1 / 2} d x\right)$, it is enough to show that there is $\varepsilon_{0}>0$ such that the operators $\mathcal{A}_{R, m, \varepsilon}-\mu$ are invertible for $|\lambda-\mu| \leq \delta_{0} / 2$ if $\varepsilon<\varepsilon_{0}$.

Assume that there are $\varepsilon_{n} \searrow 0, \mu_{n} \rightarrow \mu,|\lambda-\mu| \leq \delta_{0} / 2$, and $u_{n}$, with $\left\|u_{n}\right\|_{L^{2}\left(\Omega, g_{m}^{1 / 2} d x\right)}=1$, such that

$$
\mathcal{A}_{R, m, \varepsilon_{n}} u_{n}-\mu_{n} u_{n}=0
$$

Rewrite this as

$$
-\nabla \cdot \sigma_{R, \varepsilon_{n}} \nabla u_{n}=f_{n}=-g_{m}^{1 / 2} q u_{n}+\mu_{n} g_{m}^{1 / 2} u_{n},\left.\quad u_{n}\right|_{\partial \Omega}=0 .
$$

Taking into account the uniform boundedness of the right hand side of (112) in $L^{2}(\Omega)$, it follows from (92) and (95), with $Q=0$, that $\left\|u_{n}\right\|_{H_{0}^{1}(\Omega)} \leq C$, for some $C>0$ independent $\varepsilon_{n}$.

Thus, up to a subsequence, there exists $u \in H_{0}^{1}(\Omega)$ such that $u_{n} \rightarrow u$ as $n \rightarrow \infty$ in the weak topology of $H^{1}(\Omega)$ and strong topology of $L^{2}(\Omega)$. This shows, in particular, that $\|u\|_{L^{2}\left(\Omega, g_{m}^{1 / 2} d x\right)}=1$.

Let us show that $u$ is an eigenfunction of $\mathcal{A}_{R, m}$, see (73), corresponding to the eigenvalue $\mu$; as $|\lambda-\mu| \leq \delta_{0} / 2$ with $\lambda \in K$ and $\operatorname{dist}\left(K, \operatorname{spec}\left(\mathcal{A}_{R, m}\right)\right)>\delta>0$, this would yield a contradiction. We compare $u_{n}$ with $v_{n}$, the solution to

$$
-\nabla^{b} \cdot \sigma_{R, \varepsilon_{n}} \nabla^{b} v_{n}=\tilde{f}_{n}=-g_{m}^{1 / 2} q u+\mu g_{m}^{1 / 2} u,\left.\quad v_{n}\right|_{\partial \Omega}=0
$$

Letting $\mu_{n} \rightarrow \mu$, eq. (113) and the weak convergence $u_{n} \rightarrow u$ in $H^{1}(\Omega)$ imply that

$$
\lim _{n \rightarrow \infty}\left\|f_{n}-\tilde{f}_{n}\right\|_{L^{2}(\Omega)}=0 .
$$

Appealing again to (108), with $R>1$ fixed, we see that $u_{n}-v_{n} \rightarrow 0$ in the strong topology of $H_{0}^{1}(\Omega)$.

Using Proposition 3.1 with $Q=0$, we see that $v_{n} \rightarrow v$ in $H^{1}(\Omega)$, where

$$
-\nabla^{b} \cdot \sigma_{R} \nabla^{b} v=-g_{m}^{1 / 2} q u+\mu g_{m}^{1 / 2} u,\left.\quad v\right|_{\partial \Omega}=0
$$

Summarizing, we have that $u=v$, showing that $u$ is an eigenfunction of $\mathcal{A}_{R}$ corresponding to the eigenvalue $\lambda$. This proves claim (i).

(ii) Compare the solutions of

$$
-\nabla^{b} \cdot \sigma_{R, \varepsilon} \nabla^{b} u_{m, \varepsilon}=f_{m, \varepsilon}=f-g_{m}^{1 / 2} q u_{m, \varepsilon}+\lambda g_{m}^{1 / 2} u_{m, \varepsilon},\left.\quad u_{m, \varepsilon}\right|_{\partial \Omega}=0 .
$$


with those of

$$
-\nabla^{b} \cdot \sigma_{R} \nabla^{b} u=f_{m}=f-g_{m}^{1 / 2} q u+\lambda g_{m}^{1 / 2} u,\left.\quad u\right|_{\partial \Omega}=0 .
$$

It follows, from claim (i) together with (95), that the $u_{m, \varepsilon}$ are uniformly bounded in $H_{0}^{1}(\Omega)$. Choosing a weakly converging subsequence, we show, by similar reasoning to the above, that the limit is $u_{m}$. This proves

$$
\lim _{\varepsilon \rightarrow 0}\left(\mathscr{B}_{R, m, \varepsilon}-\lambda g_{m}^{1 / 2}\right)^{-1} f=\left(\mathscr{B}_{R, m}-\lambda g_{m}^{1 / 2}\right)^{-1} f .
$$

This, eq. (110), and the boundedness of the multiplication operator $f \mapsto g_{m}^{1 / 2} f$ in $L^{2}(\Omega)$ yields $(111)$.

For $\mu \notin \operatorname{spec}\left(\mathcal{A}_{R, m, \varepsilon}\right)$ we denote by $N_{R, m, \varepsilon}(\mu)$ the subspace of $L^{2}(\Omega)$ spanned by the eigenfunctions of $\mathcal{A}_{R, m, \varepsilon}$ corresponding to the eigenvalues in $(-\infty, \mu)$, and by $P_{R, m, \varepsilon}^{\mu}$ the orthoprojectors in $L^{2}\left(\Omega, g_{m}^{1 / 2} d x\right)$ onto $N_{R, m, \varepsilon}(\mu)$.

Lemma 3.4. Let $R>1, m \in \mathbb{Z}_{+}$, and $\mu \notin \operatorname{spec}\left(\mathcal{A}_{R, m}\right)$. (i) There is an $\varepsilon_{R, m}>0$ such that $\mu \notin \operatorname{spec}\left(\mathcal{A}_{R, m, \varepsilon}\right)$ for $0<\varepsilon<\varepsilon_{R, m}$.

(ii) For $f \in L^{2}(\Omega)$ we have

$$
\lim _{\varepsilon \rightarrow 0} P_{R, m, \varepsilon}^{\mu} f=P_{R, m}^{\mu} f,
$$

in the strong topology of $L^{2}(\Omega)$ and weak topology of $H_{0}^{1}(\Omega)$. Moreover,

$$
\lim _{\varepsilon \rightarrow 0} \operatorname{dim}\left(N_{R, m, \varepsilon}(\mu)\right)=\operatorname{dim}\left(N_{R, m}(\mu)\right) .
$$

Remark 3.5. We note that the strong convergence of the resolvents does not generally imply the stability of the resolvent set, cf. Section VIII.1.2 in [39].

Proof. (i) Take $K=\{\mu\}$ and $\delta_{0}=\operatorname{dist}\left(K, \operatorname{spec}\left(\mathcal{A}_{R, m}\right)\right)$. Lemma 3.3(i) implies that, for sufficiently small $\varepsilon, \operatorname{dist}\left(K, \operatorname{spec}\left(\mathcal{A}_{R, m, \varepsilon}\right)\right)>\delta_{0} / 2$.

(ii) Relation (114) follows immediately from (111) and the Riesz formula for the projectors, cf. eq. (62). For the proof of (115), let us consider $\lambda_{0} \in \operatorname{spec}\left(\mathcal{A}_{R, m}\right)$ and $a, b \in \mathbb{R}, a<\lambda_{0}<b$ such that $[a, b] \cap \operatorname{spec}\left(\mathcal{A}_{R, m}\right)=\left\{\lambda_{0}\right\}$. Then by (i) there are $\theta(\varepsilon), \theta(\varepsilon) \rightarrow 0$ as $\varepsilon \rightarrow 0$, such that

$$
\begin{aligned}
& \operatorname{spec}\left(\mathcal{A}_{R, m, \varepsilon}\right) \cap\left[a+\theta(\varepsilon), \lambda_{0}-\theta(\varepsilon)\right)=\emptyset, \\
& \operatorname{spec}\left(\mathcal{A}_{R, m, \varepsilon}\right) \cap\left(\lambda_{0}+\theta(\varepsilon), b-\theta(\varepsilon)\right]=\emptyset .
\end{aligned}
$$

Let $P^{\varepsilon}=P_{R, m, \varepsilon}^{b-\theta(\varepsilon)}-P_{R, m, \varepsilon}^{a+\theta(\varepsilon)}$ and $P^{0}=P_{R, m}^{b}-P_{R, m}^{a}$. Then, by (114), $\lim _{\varepsilon \rightarrow 0} P^{\varepsilon} f=P^{0} f$ in the strong topology of $L^{2}(\Omega)$ and weak topology of $H_{0}^{1}(\Omega)$.

To establish (115), it is sufficient to show that

$$
\lim _{\varepsilon \rightarrow 0} \operatorname{dim}\left(\operatorname{Ran}\left(P^{\varepsilon}\right)\right)=\operatorname{dim}\left(\operatorname{Ran}\left(P^{0}\right)\right) .
$$


Indeed, if we show eq. (116) for each eigenvalue of $\mathcal{A}_{R, m}$ smaller than $\mu$, equality (115) follows.

To show eq. (116), we note that the ranges of the orthoprojectors $P^{\varepsilon}, P^{0}$ are also subspaces of $H_{0}^{1}(\Omega) \subset L^{2}(\Omega)$. We show first that

$$
\liminf _{\varepsilon \rightarrow 0} \operatorname{dim}\left(\operatorname{Ran}\left(P^{\varepsilon}\right)\right) \geq \operatorname{dim}\left(\operatorname{Ran}\left(P^{0}\right)\right) .
$$

On the contrary, if this does not hold, there is a sequence $\varepsilon(n) \rightarrow 0$ such that

$$
\operatorname{dim}\left(\operatorname{Ran}\left(P^{\varepsilon(n)}\right)\right)<\operatorname{dim}\left(\operatorname{Ran}\left(P^{0}\right)\right)=k_{0} .
$$

Denote by $\eta_{k}, k=1, \ldots, k_{0}$, an $L^{2}(\Omega)$-orthonormal basis in $\operatorname{Ran}\left(P^{0}\right)$. (Note that as $\operatorname{Ran}\left(P^{0}\right) \subset H_{0}^{1}(\Omega)$ is finite dimensional all norms are equivalent). Introduce $\eta_{n, k}=P^{\varepsilon} \eta_{k} \in \operatorname{Ran}\left(P^{\varepsilon}\right), k=1, \ldots, k_{0}$. Consider the Gram-Schmidt matrices, $G^{n}=\left[G_{k l}^{n}\right]_{k, l=1}^{k_{0}}$

$$
G_{k l}^{n}=\int_{\Omega} \eta_{n, k}(x) \bar{\eta}_{n, l}(x) d x
$$

By (114) and orthonormality of $\eta_{k}$, the matrix $G^{n}$ is invertible for sufficiently large $n$. Thus, $\eta_{n, k}, k=1, \ldots, k_{0}$ are linearly independent. This implies that $\operatorname{dim}\left(\operatorname{Ran}\left(P^{\varepsilon(n)}\right)\right) \geq k_{0}$, contradicting (118). This proves (117).

Assume next that

$$
\limsup _{\varepsilon \rightarrow 0} \operatorname{dim}\left(\operatorname{Ran}\left(P^{\varepsilon}\right)\right)>\operatorname{dim}\left(\operatorname{Ran}\left(P^{0}\right)\right)
$$

Then there is a sequence $\varepsilon(n)$, such that

$$
\operatorname{dim}\left(\operatorname{Ran}\left(P^{\varepsilon(n)}\right)\right)>\operatorname{dim}\left(\left(\operatorname{Ran}\left(P^{0}\right)\right) .\right.
$$

Thus, there are $u_{n}$ such that

$$
\begin{gathered}
u_{n} \in \operatorname{Ran}\left(P^{\varepsilon(n)}\right), \quad\left\|u_{n}\right\|_{L^{2}\left(\Omega, g_{m}^{1 / 2} d x\right)}=1, \\
\left(u_{n}, v\right)_{L^{2}\left(\Omega, g_{m}^{1 / 2} d x\right)}=0, \quad \text { for all } v \in \operatorname{Ran}\left(P^{0}\right) .
\end{gathered}
$$

Then

$$
u_{n}=\sum_{k=1}^{k_{n}} u_{n, k} \psi_{n, k}, \quad \sum_{k=1}^{k_{n}}\left|u_{n, k}\right|^{2}=1, \quad k_{n}=\operatorname{dim}\left(\operatorname{Ran}\left(P^{\varepsilon(n)}\right)\right),
$$

where $\psi_{n, k}$ are $L^{2}\left(\Omega, g_{m}^{1 / 2} d x\right)$-orthonormal eigenfunctions of $\mathcal{A}_{R, m, \varepsilon(n)}$,

$$
\mathcal{A}_{R, m, \varepsilon(n)} \psi_{n, k}=\lambda_{n, k} \psi_{n, k}, \quad\left|\lambda_{n, k}-\lambda_{0}\right| \leq \theta(\varepsilon(n)) .
$$


Therefore,

$$
\mathcal{A}_{R, m, \varepsilon(n)} u_{n}-\lambda_{0} u_{n}=f_{n}=\sum_{k=1}^{k_{n}} u_{n, k}\left(\lambda_{n, k}-\lambda_{0}\right) \psi_{n, k} \rightarrow 0 \text { as } n \rightarrow \infty
$$

in $L^{2}\left(\Omega, g_{m}^{1 / 2} d x\right)$, so that

$$
\begin{gathered}
\int_{\Omega}\left(\sigma_{R, \varepsilon(n)} \nabla^{b} u_{n} \cdot \overline{\nabla^{b} u_{n}}+g_{m}^{1 / 2}\left(q-\lambda_{0}\right)\left|u_{n}\right|^{2}\right) d x \\
=\left(f_{n}, u_{n}\right)_{L^{2}\left(\Omega, g_{m}^{1 / 2} d x\right)} \rightarrow 0 \text { as } n \rightarrow \infty .
\end{gathered}
$$

Together with (120) and the fact that $q \in L^{\infty}(\Omega)$, this implies that the forms $\int_{\Omega} \sigma_{R, \varepsilon(n)} \nabla^{b} u_{n} \cdot \overline{\nabla^{b} u_{n}} d x$ are uniformly bounded in $n$. Recalling (95), we see that, with some $c>0$,

$$
\left\|u_{n}\right\|_{H_{0}^{1}(\Omega)}<c, \text { for all } n \in \mathbb{Z}_{+}
$$

Restricting to a subsequence of the $\varepsilon(n)$, assume there exists $u_{0} \in H_{0}^{1}(\Omega)$ such that

$$
\lim _{n \rightarrow \infty} u_{n}=u_{0} \text { weakly in } H_{0}^{1}(\Omega) \text { and strongly in } L^{2}(\Omega),
$$

so that also $\left\|u_{0}\right\|_{L^{2}\left(\Omega, g_{m}^{1 / 2} d x\right)}=1$. Let us show that $\mathcal{A}_{R, m} u_{0}=\lambda_{0} u_{0}$, contradicting the fact that $u_{0} \perp \operatorname{Ran}\left(P^{0}\right)$ in $L^{2}\left(\Omega, g_{m}^{1 / 2} d x\right)$, which follows from (120). Let $v_{n}$ be the solutions to

$$
-\nabla^{b} \cdot \sigma_{R, \varepsilon(n)} \nabla^{b} v_{n}=g_{m}^{1 / 2}\left(\lambda_{0}-q\right) u_{0},\left.\quad v_{n}\right|_{\partial \Omega}=0 .
$$

From (95), together with (122), (123), we see that $u_{n}-v_{n} \rightarrow 0$ as $n \rightarrow \infty$ in $L^{2}\left(\Omega, g_{m}^{1 / 2} d x\right)$. On the other hand, by Proposition 3.1 with $Q=0, v_{n} \rightarrow v_{0}$ in $L^{2}(\Omega)$ as $n \rightarrow \infty$, where $v_{0}$ is the solution to

$$
-\nabla^{b} \cdot \sigma_{R} \nabla^{b} v_{0}=g_{m}^{1 / 2}\left(\lambda_{0}-q\right) u_{0},\left.\quad v_{n}\right|_{\partial \Omega}=0 .
$$

Hence, $v_{0}=u_{0} \neq 0$ is an eigenfunction of $\mathcal{A}_{R, m}$ and belongs in $\operatorname{Ran}\left(P^{0}\right)$. This proves the claim.

\section{Approximate acoustic cloaking}

In this section, we show that, for $\lambda \notin \operatorname{spec}\left(\mathcal{A}_{1}\right)$, it is possible to approximate the solutions to the singular, anisotropic acoustic equation

$$
\mathcal{A}_{1} u-\lambda u=f, \quad f \in L^{2}(\Omega),
$$

by solutions to certain non-singular, isotropic acoustic equations. Namely, 
Theorem 4.1. Let $K \subset \mathbb{C}$ be a compact set such that $K \cap \operatorname{spec}\left(\mathcal{A}_{1}\right)=\emptyset$, and $\lambda \in K$. Then, for $f \in L^{2}(\Omega)$,

$$
\lim _{R \rightarrow 1} \lim _{m \rightarrow \infty} \lim _{\varepsilon \rightarrow 0}\left(\mathcal{A}_{R, m, \varepsilon}-\lambda\right)^{-1} f=\left(\mathscr{A}_{1}-\lambda\right)^{-1} f
$$

in the strong topology of $L_{g}^{2}(\Omega)$ and weak topology of $H_{g}^{1}(\Omega)$, uniformly with respect to $\lambda \in K$.

Proof. By Lemma 3.3, see eq. (111), it follows that

$$
\lim _{\varepsilon \rightarrow 0}\left(\mathcal{A}_{R, m, \varepsilon}-\lambda\right)^{-1} f=\left(\mathcal{A}_{R, m}-\lambda\right)^{-1} f
$$

in the strong topology of $L^{2}(\Omega)$ and weak topology of $H^{1}(\Omega)$, uniformly in $\lambda \in K$. Using Lemma 2.11 we obtain

$$
\lim _{m \rightarrow \infty}\left(\mathcal{A}_{R, m}-\lambda\right)^{-1} f=\left(\mathcal{A}_{R}-\lambda\right)^{-1} f
$$

in the strong topology of $H^{1}(\Omega)$, uniformly in $\lambda \in K$. Using (8) and (9), the convergences in (124) and (125) are valid also in $L_{g}^{2}(\Omega)$ and $H_{g}^{1}(\Omega)$.

As $f \in L^{2}(\Omega) \subset L_{g}^{2}(\Omega)$ we have by Lemma 2.8

$$
\lim _{R \searrow 1}\left(\mathscr{A}_{R}-\lambda\right)^{-1} f=\left(\mathscr{A}_{1}-\lambda\right)^{-1} f
$$

in the strong topology of $L_{g}^{2}(\Omega)$ and weak topology of $H_{g}^{1}(\Omega)$, uniformly with respect to $\lambda \in K$. This equation, together with (124) and (125), proves the claim.

Let us turn our attention to the convergence of the DN maps. For $R>1$ and $\varepsilon>0$, the DN map for the acoustic equation,

$$
-\nabla^{b} \cdot \sigma_{R, \varepsilon} \nabla^{b} u+g^{1 / 2} q u-\lambda g_{m}^{1 / 2} u=0,\left.\quad u\right|_{\partial \Omega}=h
$$

is the operator

$$
\Lambda_{R, \varepsilon, m}^{\lambda}:\left.h \mapsto\left(\partial_{v}+i v \cdot b\right) u_{R, \varepsilon, m}^{h}\right|_{\partial \Omega} .
$$

Here $u_{R, \varepsilon, m}^{\lambda}$ is the unique solution to (126) assuming $\lambda \notin \operatorname{spec}\left(\mathcal{A}_{R, \varepsilon, m}\right)$ and $\partial_{v}$ is the normal (radial) derivative at $\partial \Omega$.

Recall that by Lemma 2.3 the DN map $\Lambda_{\text {out }}^{\lambda}$ defined in (42) corresponds to the boundary measurements for eq. (10). In the following we assume, for technical simplicity, that $b \in C^{1}\left(\Omega, \mathbb{R}^{3}\right)$.

Corollary 4.2. Assume that the magnetic field $b$ is $C^{1}$-smooth on $\bar{\Omega}$ and $h \in$ $H^{3 / 2}(\partial \Omega),[a, b] \cap \operatorname{spec}\left(\mathcal{A}_{1}\right)=\emptyset$. Then, uniformly with respect to $\lambda \in[a, b]$,

$$
\lim _{R \rightarrow 1} \lim _{m \rightarrow \infty} \lim _{\varepsilon \rightarrow 0}\left(\Lambda_{R, m, \varepsilon}^{\lambda} h-\Lambda_{1}^{\lambda} h\right)=0,
$$

where the convergence is strong in the $H^{1 / 2}(\partial \Omega)$ topology. 
Proof. Let $h^{e} \in H^{2}(\Omega), \operatorname{supp}\left(h^{e}\right) \subset\{x: 7 / 3 \leq|x| \leq 3\}$ be an extension of $h$, that is

$$
\left.h^{e}\right|_{\partial \Omega}=h, \quad\left\|h^{e}\right\|_{H^{2}(\Omega)} \leq C, \quad \text { if }\left\|h^{e}\right\|_{H^{3 / 2}(\partial \Omega)} \leq 1 .
$$

Then the solution $u_{R, m, \varepsilon}^{\lambda}(h)$ to (127) may be represented as

$$
u_{R, m, \varepsilon}^{\lambda}(h)=h^{e}+v_{R, m, \varepsilon}^{\lambda}(h), \quad u_{1}^{\lambda}(h)=h^{e}+v_{1}^{\lambda}(h)
$$

where

$$
\begin{gathered}
v_{1}^{\lambda}=-\left(\mathcal{A}_{1}-\lambda\right)^{-1} F^{\lambda}(h), \\
v_{R, m, \varepsilon}^{\lambda}(h)=-\left(\mathcal{B}_{R, m, \varepsilon}-\lambda g_{m}^{1 / 2}\right)^{-1} F^{\lambda}(h)=-\left(\mathcal{A}_{R, m, \varepsilon}-\lambda\right)^{-1} F^{\lambda}(h), \\
F^{\lambda}(h)=-\nabla^{b} \cdot \nabla^{b} h^{e}-\lambda h^{e} \in L^{2}(\Omega), \quad \operatorname{supp}\left(F^{\lambda}(h)\right) \subset \bar{\Omega} \backslash B(7 / 3) .
\end{gathered}
$$

Here we use the fact that, in the layer $\bar{\Omega} \backslash B(7 / 3), \sigma_{R, \varepsilon}^{i j}=\sigma_{1}^{i j}=\delta^{i j}, g_{m}=g=1$.

By Theorem 4.1,

$$
\lim _{R \rightarrow 1} \lim _{m \rightarrow \infty} \lim _{\varepsilon \rightarrow 0}\left\|v_{R, m, \varepsilon}^{\lambda}(h)-v_{1}^{\lambda}(h)\right\|_{L^{2}(\{7 / 3 \leq|x| \leq 3\})}=0 .
$$

It then follows from Theorem 9.13 in [23] that

$$
\lim _{R \rightarrow 1} \lim _{m \rightarrow \infty} \lim _{\varepsilon \rightarrow 0}\left\|v_{R, m, \varepsilon}^{\lambda}(h)-v_{1}^{\lambda}(h)\right\|_{H^{2}(\{8 / 3 \leq|x| \leq 3\})}=0,
$$

so that, by the trace theorem for Sobolev spaces, we have

$$
\left.\lim _{R \rightarrow 1} \lim _{m \rightarrow \infty} \lim _{\varepsilon \rightarrow 0}\left(\partial_{v}+i v \cdot b\right)\left(u_{R, m, \varepsilon}^{\lambda}(h)-u_{1}^{\lambda}(h)\right)\right|_{\partial \Omega}=0,
$$

strongly in $H^{1 / 2}(\partial \Omega)$.

Our next goal is to show the existence of a sequence of non-singular isotropic mass densities and non-singular bulk moduli, uniformly bounded (in $n$ ) from above but not from below, with the corresponding acoustic operators approximating the singular acoustic operator $\mathcal{A}_{1}-\lambda$.

Theorem 4.3. There exist sequences $R(n) \rightarrow 1, m(n) \rightarrow \infty$, and $\varepsilon(n) \rightarrow 0$ such that, for any $f \in L^{2}(\Omega), \lambda \in \mathbb{C}, \mu \in \mathbb{R}, \lambda, \mu \notin \operatorname{spec}\left(\mathscr{A}_{1}\right)$,

$$
\begin{gathered}
\lim _{n \rightarrow \infty}\left(\mathcal{A}_{R(n), m(n) \varepsilon(n)}-\lambda\right)^{-1} f=\left(\mathcal{A}_{1}-\lambda\right)^{-1} f \text { in } L_{g}^{2}(\Omega), \\
\lim _{n \rightarrow \infty} P_{R(n), m(n), \varepsilon(n)}^{\mu} f=P_{1}^{\mu} f \text { in } L_{g}^{2}(\Omega), \\
\lim _{n \rightarrow \infty} \operatorname{dim}\left(N_{R(n), m(n), \varepsilon(n)}(\mu)\right)=\operatorname{dim}\left(N_{1}(\mu)\right) .
\end{gathered}
$$

For compact $K \subset \mathbb{C}$ with $K \cap \operatorname{spec}(\mathcal{A})=\emptyset$, for $n$ sufficiently large one has $K \cap$ $\operatorname{spec}\left(\mathcal{A}_{R(n), m(n) \varepsilon(n)}\right)=\emptyset$ and the limits are uniform in $\lambda \in K$ and $\mu \in K \cap \mathbb{R}$. 
Proof. Let $\left\{f_{p}: p=1,2, \ldots\right\}$ be a dense set in $L^{2}(\Omega)$. Let $K_{n} \subset \mathbb{C}, n=1,2, \ldots$, be a sequence of compact sets so that

$$
\begin{gathered}
K_{n} \cap \operatorname{spec}\left(\mathcal{A}_{1}\right)=\emptyset, \quad K_{n} \subset K_{n+1}^{\mathrm{int}}, \\
\cup_{n=1}^{\infty} K_{n}=\mathbb{C} \backslash \operatorname{spec}\left(\mathcal{A}_{1}\right), \quad \operatorname{dist}\left(\operatorname{spec}\left(\mathcal{A}_{1}\right), K_{n}\right) \geq n^{-1 / 2} .
\end{gathered}
$$

As $L^{2}(\Omega) \subset L_{g}^{2}(\Omega)$, it follows from Lemmas 2.8 and 2.9 , that, for any $n \in \mathbb{Z}_{+}$, there exists a $R(n)>1$ such that

$$
\begin{gathered}
\left\|\left(\mathcal{A}_{R(n)}-\lambda\right)^{-1} f_{p}-\left(\mathcal{A}_{1}-\lambda\right)^{-1} f_{p}\right\|_{g}<\frac{1}{n}, \\
\operatorname{dim}\left(N_{R(n)}(\mu)\right)=\operatorname{dim}\left(N_{1}(\mu)\right), \quad \operatorname{dist}\left(\operatorname{spec}\left(\mathcal{A}_{R(n)}\right), K_{n}\right) \geq \frac{n^{-1 / 2}}{2}, \\
\left\|P_{R(n)}^{\mu}-P_{1}^{\mu}\right\|_{L_{g}^{2} \rightarrow L_{g}^{2}} \leq \frac{1}{n}, \quad\left\|P_{R(n)}^{\mu} f_{p}-P_{1}^{\mu} f_{p}\right\|_{L_{g}^{2}} \leq \frac{1}{n},
\end{gathered}
$$

for all $p=1, \ldots, p_{n}, \lambda \in K_{n}, \mu \in K_{n} \cap \mathbb{R}$. Here $p_{n}$ is defined so that

$$
\left\{f \in L^{2}(\Omega):\|f\|_{L^{2}(\Omega)} \leq n\right\} \subset N_{1 / n}\left(\left\{f_{p}\right\}_{p=1}^{p_{n}}\right),
$$

where $N_{\varepsilon}(S)$ denotes the $\varepsilon$-neighborhoods of $S$ in $L^{2}(\Omega)$.

Having chosen $R(n)$, using (8) and Lemma 2.11 and Corollary 2.12, we choose $m(n)$ such that

$$
\begin{gathered}
\left\|\left(\mathcal{A}_{R(n), m(n)}-\lambda\right)^{-1} f_{p}-\left(\mathcal{A}_{R(n)}-\lambda\right)^{-1} f_{p}\right\|_{g}<\frac{1}{n}, \\
\operatorname{dim}\left(N_{R(n), m(n)}(\mu)\right)=\operatorname{dim}\left(N_{1}(\mu)\right), \\
\operatorname{dist}\left(\operatorname{spec}\left(\mathcal{A}_{R(n), m(n)}\right), K_{n}\right) \geq \frac{n^{-1 / 2}}{3}, \\
\left\|P_{R(n), m(n)}^{\mu}-P_{R(m)}^{\mu}\right\|_{L^{2} \rightarrow L_{g}^{2}} \leq \frac{1}{n}, \\
\left\|P_{R(n), m(n)}^{\mu} f_{p}-P_{R(n)}^{\mu} f_{p}\right\|_{L_{g}^{2}} \leq \frac{1}{n},
\end{gathered}
$$

for all $p=1, \ldots, p_{n}, \lambda \in K_{n}, \mu \in K_{n} \cap \mathbb{R}$. Having chosen $R(n)$ and $m(n)$, one can now use (8) and Lemmas 3.3 and 3.4 to choose $\varepsilon(n)>0$ so that,

$$
\begin{gathered}
\left\|\left(\mathcal{A}_{R(n), m(n), \varepsilon(n)}-\lambda\right)^{-1} f_{p}-\left(\mathcal{A}_{R(n), m(n)}-\lambda\right)^{-1} f_{p}\right\|_{g}<\frac{1}{n}, \\
\operatorname{dim}\left(N_{R(n), m(n), \varepsilon(n)}(\mu)\right)=\operatorname{dim}\left(N_{R(n), m(n)}(\mu)\right), \\
\operatorname{dist}\left(\operatorname{spec}\left(\mathcal{A}_{R(n), m(n), \varepsilon(n)}\right), K_{n}\right) \geq \frac{n^{-1 / 2}}{4}, \\
\left\|P_{R(n), m(n), \varepsilon(n)}^{\mu} f_{p}-P_{R(n), m(n)}^{\mu} f_{p}\right\|_{L_{g}^{2}} \leq \frac{1}{n},
\end{gathered}
$$


for all $p=1, \ldots, p_{n}, \lambda \in K_{n}$ and $\mu \in K_{n} \cap \mathbb{R}$, and,

$$
\left.\left\|\left(\mathcal{A}_{R, m, \varepsilon}-\lambda\right)^{-1}\right\|_{L^{2}\left(\Omega, g_{m}^{1 / 2} d x\right.}\right) \rightarrow L^{2}\left(\Omega, g_{m}^{1 / 2} d x\right) \leq 6 n^{1 / 2},
$$

with $\lambda \in K_{n}$.

Clearly, eq. (136), (137) and (138) imply that

$$
\begin{gathered}
\left\|\left(\mathcal{A}_{R(n), m(n), \varepsilon(n)}-\lambda\right)^{-1} f_{p}-\left(\mathcal{A}_{1}-\lambda\right)^{-1} f_{p}\right\|_{g}<\frac{3}{n} \\
\operatorname{dim}\left(N_{R(n), m(n), \varepsilon(n)}(\mu)\right)=\operatorname{dim}\left(N_{1}(\mu)\right) \\
\left\|P_{R(n), m(n), \varepsilon(n)}^{\mu} f_{p}-P_{1}^{\mu} f_{p}\right\|_{L_{g}^{2}} \leq \frac{3}{n}
\end{gathered}
$$

for all $p=1, \ldots, p_{n}, \lambda \in K_{n}, \mu \in K_{n} \cap \mathbb{R}$.

In particular, (140) implies (134) due to $\cup_{n} K_{n}=\mathbb{C} \backslash \operatorname{spec}\left(\mathcal{A}_{1}\right)$. It remains to show that, for $f \in L^{2}(\Omega)$ and a compact set $K$ such that $K \cap \operatorname{spec}\left(\mathcal{A}_{1}\right)=\emptyset$, one has that $\left(\mathcal{A}_{R(n), m(n), \varepsilon(n)}-\lambda\right)^{-1} f$ converge to $\left(\mathcal{A}_{1}-\lambda\right)^{-1} f$ in $L_{g}^{2}(\Omega)$ and that convergence is uniform for all $\lambda \in K$. Indeed, this will provide eq. (132). Using the Riesz formula for $P_{R(n), m(n), \varepsilon(n)}^{\mu}, P_{1}^{\mu}$, this will also prove equation (133).

Let $n_{0} \in \mathbb{Z}_{+}$be such that $K \subset K_{n}$ for all $n \geq n_{0}$. For $n \geq n_{0}$, there exists $f_{p(n)}$ with $p(n) \leq p_{n}$ such that

$$
\left\|f-f_{p(n)}\right\|_{L^{2}(\Omega)}<\frac{1}{n}
$$

Clearly, for $\lambda \in K$,

$$
\begin{aligned}
&\left\|\left(\mathcal{A}_{R(n), m(n), \varepsilon(n)}-\lambda\right)^{-1} f-\left(\mathcal{A}_{1}-\lambda\right)^{-1} f\right\|_{g} \\
& \leq\left\|\left(\mathcal{A}_{R(n), m(n), \varepsilon(n)}-\lambda\right)^{-1} f_{p(n)}-\left(\mathcal{A}_{1}-\lambda\right)^{-1} f_{p(n)}\right\|_{g} \\
&+\left\|\left(\mathcal{A}_{R(n), m(n), \varepsilon(n)}-\lambda\right)^{-1}\left(f_{p(n)}-f\right)\right\|_{g} \\
&+\left\|\left(\mathcal{A}_{1}-\lambda\right)^{-1}\left(f_{p(n)}-f\right)\right\|_{g} .
\end{aligned}
$$

By (140), the first term in the right-hand side of (142) is bounded by $3 / n$. To estimate the second term, we use the estimates (66), (139), and (141), which imply that this term $\mathrm{s}$ bounded by $12(2 / n)^{1 / 2}$ for $n \geq n_{0}$. Finally, by (8) and (135),

$$
\left\|\left(\mathcal{A}_{1}-\lambda\right)^{-1}\right\|_{L^{2} \rightarrow L_{g}^{2}} \leq \sqrt{8}\left\|\left(\mathcal{A}_{1}-\lambda\right)^{-1}\right\|_{L_{g}^{2} \rightarrow L_{g}^{2}} \leq \frac{\sqrt{8}}{\operatorname{dist}\left(\lambda, \operatorname{spec}\left(\mathcal{A}_{1}\right)\right)} \leq \sqrt{8 n},
$$

for $\lambda \in K$. Thus by (141), the third term on the right-hand side of (142) is bounded by $(8 / n)^{1 / 2}$ for $n \geq n_{0}$.

Summarizing, we see that the left-hand side of (142) tends to 0 , as $n \rightarrow \infty$, uniformly for $\lambda \in K$, thus proving (132). 
In the sequel, we will use abbreviated notations

$$
\sigma_{(n)}=\sigma_{R(n), \varepsilon(n)}, \quad g_{(n)}=g_{m(n)}, \quad \mathcal{A}_{(n)}=\mathcal{A}_{R(n), m(n), \varepsilon(n)}
$$

for the sequences $R(n), m(n), \varepsilon(n)$ obtained in Theorem 4.3. For simplicity, denote by $\Lambda_{(n)}^{\lambda}$, rather than $\Lambda_{R(n), m(n), \varepsilon(n)}^{\lambda}$, the corresponding DN map.

Regarding the convergence of the DN-maps, by the same arguments as in proof of Corollary 4.2, we obtain

Corollary 4.4. Assume that the magnetic field $b$ is $C^{1}$-smooth in $\bar{\Omega}$. Let $K \subset \mathbb{C}$ be compact and such that $K \cap \operatorname{spec}\left(\mathcal{A}_{1}\right)=\emptyset$. Then, for any $h \in H^{3 / 2}(\partial \Omega)$,

$$
\lim _{n \rightarrow \infty} \Lambda_{(n)}^{\lambda} h=\Lambda_{\text {out }}^{\lambda} h
$$

where the limit is in $H^{1 / 2}(\partial \Omega)$, uniformly with respect to $\lambda \in K$.

In particular, when $b$ and $\left.q\right|_{\Omega \backslash B(1)}$ vanish, $\Lambda_{\text {out }}^{\lambda}$ corresponds to the measurements on the boundary of a homogeneous ball. Thus (144) means that the isotropic material parameters $\sigma_{(n)}$ and $g_{(n)}$ approximate an acoustic invisibility cloak as $n \rightarrow \infty$.

\section{Approximate quantum cloaking}

The results of the previous sections can now be used to obtain approximate quantum cloaking at a fixed energy, for any potential $Q \in L^{\infty}(\Omega)$ supported inside the cloaked region $B(1)$.

In sequel, we assume that the conductivities $\sigma_{R}$ satisfy (23) and that also $\sigma_{R} \in$ $C^{2}\left(\bar{\Omega} ; \mathbb{R}^{2}\right)$. This yields that also $\sigma_{R, \varepsilon}, \sigma_{(n)} \in C^{2}\left(\bar{\Omega} ; \mathbb{R}^{2}\right)$.

Let $E \in \mathbb{R}$ be a given energy level and

$$
q^{E}(x)=\frac{1}{4} Q(x)+\frac{3}{4} E \chi_{1}(x),
$$

where $\chi_{1}$ is the indicator function of the ball $B(1)$. In this section we assume that $E$ and $Q$ are such that $E$ is not in the spectrum of the operator $\mathcal{A}_{1}=\mathcal{A}_{1}^{E}$ defined in (19) using the potential $q^{E}$. Observe that $\operatorname{spec}\left(\mathcal{A}_{1}^{E}\right)=\operatorname{spec}\left(A_{\text {out }}\right) \cup \operatorname{spec}\left(A_{\text {in }}^{E}\right)$, where $A_{\text {out }}$ and $A_{\text {in }}^{E}$ are defined by (40) in (41) using potential $q^{E}$. Note that $A_{\text {out }}$ is independent of $E$ and $A_{\text {in }}^{E}=\frac{1}{4} S_{\text {in }}+\frac{3}{4} E$, where $S_{\text {in }}$ is the Schrödinger operator

$$
S_{\text {in }}=-\Delta+Q, \mathscr{D}\left(S_{\text {in }}\right)=\left\{v_{2} \in H^{2}(B(1)):\left.\partial_{\nu} v\right|_{\partial B(1)}=0\right\} .
$$

Observe that

$$
E \notin \operatorname{spec}\left(\mathcal{A}_{1}^{E}\right) \quad \Longleftrightarrow \quad E \notin \operatorname{spec}\left(S_{\text {in }}\right) \text { and } E \notin \operatorname{spec}\left(A_{\text {out }}\right) \text {. }
$$


Then, for $n$ large enough, $E \notin \operatorname{spec}\left(\mathcal{A}_{(n)}\right)$. Next, consider the solutions $u_{n}$ of

$$
\left(-g_{(n)}^{-1 / 2} \nabla^{b} \cdot \sigma_{(n)} \nabla^{b}+q^{E}-E\right) u_{n}=0 \text { in } \Omega,\left.\quad u_{n}\right|_{\partial \Omega}=h .
$$

This equation can be converted to a Schrödinger equation with magnetic potential using the gauge transformation,

$$
\psi_{n}(x)=\sigma_{(n)}^{1 / 2}(x) u_{n}(x)
$$

Then

$$
-\sigma_{(n)}^{-1 / 2} \nabla^{b} \cdot \sigma_{(n)} \nabla^{b}\left(\sigma_{(n)}^{-1 / 2} \psi_{n}\right)=-\nabla^{b} \cdot \nabla^{b} \psi_{n}+W_{(n)} \psi_{n},
$$

where

$$
W_{(n)}=\sigma_{(n)}^{-1 / 2} \Delta\left(\sigma_{(n)}^{1 / 2}\right), \quad \operatorname{supp}\left(W_{(n)}\right) \subset \overline{B(2)} \backslash B(1) .
$$

Thus, using the transformation (148) we see that the acoustic eq. (147) for $u_{n}$ is equivalent to the Schrödinger equation for $\psi_{n}$,

$$
\left(-\nabla^{b} \cdot \nabla^{b}+W_{(n)}+\frac{g_{(n)}^{1 / 2}}{\sigma_{(n)}}\left(q^{E}-E\right)\right) \psi_{n}=0,\left.\quad \psi_{n}\right|_{\partial \Omega}=h,
$$

where for the boundary condition we use $\left.\psi_{n}\right|_{\partial \Omega}=\left.u_{n}\right|_{\partial \Omega}=h$, since $\sigma_{(n)}=1$ near $\partial \Omega$.

Next, define the cloaking potential

$$
\begin{aligned}
V_{n}^{E}(x) & =W_{(n)}(x)+\frac{g_{(n)}^{1 / 2}(x)}{\sigma_{(n)}(x)}\left(\frac{3 E}{4} \chi_{1}(x)-E\right)+E \\
& =W_{(n)}(x)+E\left(1-\chi_{1}(x)\right)\left(1-\frac{g_{(n)}^{1 / 2}(x)}{\sigma_{(n)}(x)}\right),
\end{aligned}
$$

where we have used the fact that $g_{(n)}^{1 / 2}(x)=8, \sigma_{(n)}(x)=2$ for $|x|<1$. Thus $V_{n}^{E}=0$ in $B(1)$. Clearly, $V_{n}^{E}$ vanishes also near $\partial \Omega$. Then (151) can be written as

$$
\left(-\nabla^{b} \cdot \nabla^{b}+V_{n}^{E}(x)+Q-E\right) \psi_{n}=0,\left.\quad \psi_{n}\right|_{\partial \Omega}=h .
$$

Now we are ready to prove our main result concerning approximate cloaking in quantum mechanics.

Theorem 5.1. Assume that $Q \in L^{\infty}(\Omega)$ is a function supported in $B(1), b \in$ $C^{1}\left(\Omega, \mathbb{R}^{3}\right)$, and $E \in \mathbb{R}$ are such that $E \notin \operatorname{spec}\left(A_{\text {out }}\right) \cup \operatorname{spec}\left(S_{\text {in }}\right)$. Then for any $h \in H^{3 / 2}(\partial \Omega)$,

$$
\lim _{n \rightarrow \infty} \Lambda_{V_{n}^{E}+Q}^{E} h=\Lambda_{\text {out }}^{E} h \text { in } H^{1 / 2}(\partial \Omega)
$$


Here $\Lambda_{V_{n}^{E}+Q}^{E}$ are the DN maps,

$$
\Lambda_{V_{n}^{E}+Q}^{E}:\left.h \mapsto \partial_{\nu} \psi_{n}\right|_{\partial \Omega}, \quad\left(-\nabla^{b} \cdot \nabla^{b}+V_{n}^{E}+Q-E\right) \psi_{n}=0,\left.\quad \psi_{n}\right|_{\partial \Omega}=h,
$$

while the DN-map $\Lambda_{\text {out }}^{E}$ corresponds to the operator $A_{\text {out }}$ with $\kappa_{1}=0$, see (40) and (42).

Proof. By the hypotheses of the theorem, it follows that $E \notin \operatorname{spec}\left(\left(\mathcal{A}_{1}^{E}\right)\right.$, where $\mathcal{A}_{1}$ defined by (19) with magnetic potential $b$ and the potential $q^{E}$ given by (145). Thus, the Dirichlet problem (147) is uniquely solvable for large $n$. As the gauge transformation (148) is the identity map near $\partial \Omega$, we see that $\left.\partial_{\nu} \psi_{n}\right|_{\partial \Omega}=\left.\partial_{\nu} u_{n}\right|_{\partial \Omega}$ and $\left.\psi_{n}\right|_{\partial \Omega}=\left.u_{n}\right|_{\partial \Omega}$. The DN maps for the Schrödinger eqution (153) and for eq. (147) thus coincide, and the assertion follows from Corollary 4.4 .

Note that Theorem 5.1 is of a very different nature than the well-known results from the classical theory of spectral convergence, since the cloaking potentials $V_{n}^{E}$ do not tend to 0 as $n \rightarrow \infty$. On the contrary, as seen from the construction of $\sigma_{R, \varepsilon}$ in Section 3.2 and definition (150) and (152), $\sup _{x}\left|V_{n}^{E}(x)\right| \rightarrow \infty$ as $n \rightarrow \infty$. Moreover, $V_{n}^{E}$ is of a highly oscillatory nature in $B(2) \backslash B(1)$ with quasiperiod tending to 0 as $n \rightarrow \infty$.

Theorem 5.1 has two important physical consequences; see [29] and [30] for further discussion and applications. Consider separately the following two cases. (i) Suppose that $b=0$. Since $Q$ is supported in $\bar{B}(1)$, the operator $A_{\text {out }}=-\Delta$ is the free Schrödinger operator. Then, from a physical point of view, the potentials $V_{n}^{E}+Q$ can be considered as almost transparent potentials at energy $E$. Also, the $V_{n}^{E}$, which depend on $E$ but are independent of $Q$, serve as approximate invisibility cloaks for two-body scattering in quantum mechanics. As all measurement devices have limited precision, we can interpret this as saying that, given a specific device, one can design, for a given energy level $E$, a potential to cloak an object, i.e. an arbitrary potential, from any single-particle measurements made at this energy $E$.

(ii) Now suppose that $Q=0$, while $b \in C^{1}\left(\Omega, \mathbb{R}^{3}\right), b \neq 0$. We now have $A_{\text {out }}=-\nabla^{\beta_{1}} \cdot \nabla^{\beta_{1}}$, and, due to the transformation rules for the magnetic potentials, $\beta_{1}$ is in general no longer bounded near $O$. Thus, the potentials $V_{n}^{E}$ act as devices which give an external observer the illusion that, as $n \rightarrow \infty$, the magnetic field is unbounded near $O$. In particular, for $b(x)=B_{0} \times x, B_{0} \in \mathbb{R}^{3}$, corresponding to a homogeneous magnetic field, the illusion $\beta_{1}(x)$ has a singularity of the order $|x|^{-1}$ at $O$; see [29] for details.

\section{DN map near exceptional values of $E$}

Theorem 5.1 shows that the behavior of $\Lambda_{V_{n}^{E}+Q}^{E}$, when $E$ is far from $\operatorname{spec}\left(\mathcal{A}_{\text {in }}^{E}\right)$ and $n$ is large, well approximates the behavior of $\Lambda_{\text {out }}^{E}$. This situation changes dramatically 
when $E$ is close to an eigenvalue of the cloaked region.

For simplicity, let us consider the case of an $E^{0} \in \mathbb{R}$ which, while being in the resolvent set of $A_{\text {out }}$, is also a simple eigenvalue of $A_{\text {in }}^{E^{0}}$, that is $E^{0} \in \operatorname{spec}\left(S_{\text {in }}\right)$, see (146). This implies that $E^{0}$ is a simple eigenvalue of $\mathcal{A}_{1}^{E^{0}}$. The corresponding eigenfunction $u^{0}$ then satisfies $\operatorname{supp}\left(u^{0}\right) \subset \bar{B}(1)$, i.e. $u^{0}$ is a trapped state supported in the cloaked region, cf. Lemma 2.3 and (43).

In the following, let $d \in(0,1)$ be such that $\operatorname{dist}\left(E^{0}, \operatorname{spec}\left(\mathcal{A}_{1}^{E^{0}}\right) \backslash E^{0}\right)>d$.

Theorem 6.1. Let $Q \in L^{\infty}(\Omega)$ be a function supported in $\bar{B}(1), b \in C^{1}\left(\Omega, \mathbb{R}^{3}\right)$, and $E^{0} \in \mathbb{R}$ be an eigenvalue of $\mathcal{A}_{1}^{E^{0}}$ corresponding to potentials $b$ and $q^{E_{0}}$ defined in (145). Assume that $E^{0}$ has multiplicity one, and let $u^{0}$ be the corresponding eigenfunction supported in $\bar{B}(1)$.

(i) There is a sequence $E_{(n)}, E_{(n)} \rightarrow E^{0}$ as $n \rightarrow \infty$, such that $E_{(n)}$ are simple Dirichlet eigenvalues of the Schrödinger operators $-\nabla^{b} \cdot \nabla^{b}+V_{(n)}^{E_{(n)}}+Q$. Moreover, the $L^{2}(\Omega)$-normalized eigenfunctions $\varphi_{(n)}$ of these Schrödinger operators for the eigenvalues $E_{(n)}$ satisfy, for any $\rho>2$,

$$
\left.\lim _{n \rightarrow \infty} \varphi_{(n)}\right|_{\bar{\Omega} \backslash B(\rho)}=0, \quad \text { in } C^{1}(\bar{\Omega} \backslash B(\rho)) .
$$

(ii) Let $h \in H^{3 / 2}(\partial \Omega)$ and $\psi_{(n)}(h)$ be the solution to (153) for some $E$ with $0<\left|E-E_{(n)}\right|<d / 2$. Then

$$
\begin{aligned}
\left.\left(\partial_{v}+i v \cdot b\right) \psi_{(n)}(h)\right|_{\partial \Omega} & =\left.\frac{\alpha_{(n)}^{0}(h)}{E-E_{(n)}}\left(\partial_{v}+i v \cdot b\right) \psi_{(n)}\right|_{\partial \Omega}+p_{(n)}^{E}(h), \\
\alpha_{(n)}^{0}(h) & =\int_{\partial \Omega} h \frac{\partial \overline{\psi(n)}}{\partial v} d S,
\end{aligned}
$$

and functions $p_{(n)}^{E}(h)$ are uniformly bounded, as $n \rightarrow \infty$, in $\left.H^{1 / 2}(\partial \Omega)\right)$ for $\|h\|_{H^{3 / 2}}$ $\leq 1$ and $E \in\left(E^{0}-d / 4, E^{0}+d / 4\right)$.

Proof. For a given potential $Q$, the potential $q^{E}$ defined in (145) depends on $E$. Thus we start by analyzing how the eigenvalues and eigenfunctions of $\mathcal{A}_{1}^{E}$ and $\mathcal{A}_{(n)}^{E}$ change relative to the variation of $E$. Denote by $\lambda(k, E), k=1,2, \ldots$, the eigenvalues of $\mathcal{A}_{1}^{E}$, numbered in increasing order and taking multiplicity into account. The dependence of these eigenvalues on $E$ then follows from (146). Similarly, let $\lambda_{(n)}(k, E)$ be the eigenvalues of $\mathcal{A}_{(n)}^{E}$, where $\mathcal{A}_{(n)}^{E}$ is the operator of form (143) with $q=q^{E}$. Observe that

$$
\mathcal{A}_{1}^{E}=\mathcal{A}_{1}^{E^{0}}+\left(q^{E}-q^{E^{0}}\right), \quad \mathcal{A}_{(n)}^{E}=\mathcal{A}_{(n)}^{E^{0}}+\left(q^{E}-q^{E^{0}}\right) .
$$

Using Kato-Rellich formula, see Theorem VII.3.6 in [39], and the fact that $\| q^{\widetilde{E}}-$ 
$q^{E} \|_{L^{\infty}}=\frac{3}{4}|\widetilde{E}-E|$, see $(145)$, we obtain that

$$
\left|\lambda_{(n)}(k, \widetilde{E})-\lambda_{(n)}(k, E)\right| \leq \frac{3}{4}|\widetilde{E}-E| .
$$

In the future we will consider only the value $k=k^{0}$ such that $\lambda\left(k^{0}, E^{0}\right)=E^{0}$, writing, e.g., $\lambda_{(n)}(E)$ for $\lambda_{(n)}\left(k^{0}, E\right)$. Next, consider the spectral projectors for $\mu=E^{0}-d / 2$ or $\mu=E^{0}+d / 2$. Let $P_{1}^{\mu}(E)$ be the Riesz projectors for the operators $\mathcal{A}_{1}^{E}$ and $P_{(n)}^{\mu}(E)$ be the projectors for $\mathcal{A}_{(n)}^{E}$. They are defined analogously to (62) using a contour $\Gamma \subset \mathbb{C}$ that surrounds all of the eigenvalues smaller than $\mu$. We can assume that $\Gamma$ is such a contour that, for $n$ large enough, the distance from $\Gamma$ to the eigenvalues of the operators $\mathcal{A}_{1}^{E^{0}}$ and $\mathcal{A}_{(n)}^{E^{0}}$ is more than $d / 4$. Then the norm of $\left(\mathcal{A}_{(n)}^{E^{0}}-z\right)^{-1}$ in $L^{2}\left(\Omega, g_{(n)}^{1 / 2} d x\right)$ is bounded by $4 / d$. Thus, assuming that $\left|E-E^{0}\right|<d / 8$, we obtain, using the formula

$\left(\mathcal{A}_{(n)}^{E}-z\right)^{-1}-\left(\mathcal{A}_{(n)}^{E^{0}}-z\right)^{-1}=\left(\mathcal{A}_{(n)}^{E^{0}}-z\right)^{-1}\left(\left(I-\left(q^{E}-q^{E^{0}}\right)\left(\mathcal{A}_{(n)}^{E^{0}}-z\right)^{-1}\right)^{-1}-I\right)$

in (62), the estimate

$$
\left\|P_{(n)}^{\mu}(E)-P_{(n)}^{\mu}\left(E^{0}\right)\right\|_{L^{2}\left(g_{(n)}^{1 / 2} d x\right) \rightarrow L^{2}\left(g_{(n)}^{1 / 2} d x\right)} \leq C_{\mu}\left|E-E^{0}\right|,
$$

where $C_{\mu}$ depends only on the choice of $\Gamma$.

As $E^{0}$ is the only eigenvalue of $\mathcal{A}_{1}^{E^{0}}$ in the interval $\left(E^{0}-d, E^{0}+d\right)$ and has the multiplicity one, it follows from Theorem 4.3 that, when $n$ is large enough, then $\mathcal{A}_{(n)}^{E^{0}}$ has only one eigenvalue $E_{n}^{0}$ in the interval $\left(E^{0}-3 d / 4, E^{0}+3 d / 4\right)$ and $\left|E_{n}^{0}-E^{0}\right|<d / 4$. Moreover, the eigenvalue $E_{n}^{0}$ is simple.

Let us show that there are $E_{(n)}, E_{(n)} \rightarrow E^{0}$, such that

$$
\lambda_{(n)}\left(E_{(n)}\right)=E_{(n)} .
$$

Observe that, by Theorem $4.3, \lambda_{(n)}\left(E^{0}\right) \rightarrow E^{0}$ as $n \rightarrow \infty$. Together with (159) this implies that for any $\varepsilon>0$ there is $n(\varepsilon)$ such that for $n>n(\varepsilon)$,

$$
\lambda_{(n)}(\cdot):\left[E^{0}-\varepsilon, E^{0}+\varepsilon\right] \rightarrow\left[E^{0}-\varepsilon, E^{0}+\varepsilon\right] .
$$

As $\lambda_{(n)}(\cdot)$ is a contraction, see (159), we conclude by the Banach fixed point theorem that there is a unique $E_{(n)}$ satisfying (161).

Returning to eq. (160) we see that

$$
\lim _{n \rightarrow \infty}\left\|P_{(n)}^{\mu}\left(E_{(n)}\right)-P_{(n)}^{\mu}\left(E^{0}\right)\right\|_{L^{2}\left(g_{(n)}^{1 / 2} d x\right) \rightarrow\left(g_{(n)}^{1 / 2} d x\right)}=0,
$$

Combining this with (133) (for $q=q^{E^{0}}$ ) and embedding (66), we see

$$
\lim _{n \rightarrow \infty} P_{(n)}^{\mu}\left(E_{(n)}\right) f=P_{1}^{\mu}\left(E^{0}\right) f \quad \text { in } L_{g}^{2}(\Omega) .
$$


Let $\mu=E+d / 2$ and $v=E-d / 2$. For large $n$, the operators $P_{(n)}^{\mu}\left(E_{(n)}\right)-$ $P_{(n)}^{v}\left(E_{(n)}\right)$ are the orthoprojectors in $L^{2}\left(\Omega, g_{(n)}^{1 / 2} d x\right)$ onto the eigenspace of $\mathcal{A}_{(n)}^{E_{(n)}}$ corresponding to the eigenvalue $E_{(n)}$. Using (162) we see that, for $n$ large enough, the eigenvalue $E_{(n)}$ has multiplicity one. Denote by $\tilde{u}_{(n)}$ the eigenfunction corresponding to $E_{(n)}$, normalized in $L_{g}^{2}(\Omega)$. Now $u^{0}$ is supported in $B(1)$ and thus

$$
\left\|u^{0}\right\|_{g}=\left\|u^{0}\right\|_{L^{2}\left(\Omega, g_{(n)}^{1 / 2} d x\right)}=1, n>0, \quad\left\|u^{0}\right\|_{L^{2}(\Omega)}=\frac{1}{\sqrt{8}} .
$$

Using (163) we see that

$$
\left(P_{(n)}^{\mu}\left(E_{(n)}\right)-P_{(n)}^{v}\left(E_{(n)}\right)\right) u^{0}=a_{n} \tilde{u}_{(n)} \underset{n \rightarrow \infty}{\longrightarrow}\left(P_{1}^{\mu}\left(E^{0}\right)-P_{1}^{v}\left(E^{0}\right)\right) u^{0}=u^{0},
$$

in $L_{g}^{2}(\Omega)$, where $a_{n}=\left|a_{n}\right| e^{i \alpha_{n}}$ and $\left|a_{n}\right| \rightarrow 1$ as $n \rightarrow \infty$.

Denoting $u_{(n)}=e^{i \alpha_{n}} \tilde{u}_{(n)}$, we see that

$$
\lim _{n \rightarrow \infty} u_{(n)}=u^{0} \text { in } L_{g}^{2}(\Omega) .
$$

Since $u^{0}(x)=0,|x|>1$, this implies

$$
\lim _{n \rightarrow \infty}\left\|\left.u_{(n)}\right|_{\Omega \backslash B(2)}\right\|_{L^{2}(\Omega \backslash B(2))}=0 .
$$

Observe that $\sigma_{(n)}=\gamma_{0}, g_{(n)}=1$ and $V_{(n)}^{E}=0$ in $\Omega \backslash B(2)$. Thus it follows from (165) that the functions $u_{(n)}$ satisfy

$$
-\nabla^{b} \cdot \nabla^{b} u_{(n)}=E_{(n)} u_{(n)} \quad \text { in } \Omega \backslash B(2),\left.\quad u_{(n)}\right|_{\partial \Omega}=0,
$$

where the right side $E_{(n)} u_{(n)} \rightarrow 0$ in $L^{2}(\Omega \backslash B(2))$ as $n \rightarrow \infty$. Since $b \in C^{1}(\Omega \backslash$ $B(2))$, standard elliptic regularity results [23] imply that

$$
\lim _{n \rightarrow \infty}\left\|u_{(n)}\right\|_{C^{1}(\Omega \backslash B(\rho))}=0,
$$

for any $2<\rho<3$. Using the transformation (148) to define $\varphi_{n}(x)=\sigma_{(n)}^{1 / 2}(x) u_{n}(x)$, we see that

$$
\left(-\nabla^{b} \cdot \nabla^{b}+V_{n}^{E_{(n)}}+Q\right) \varphi_{n}=E_{(n)} \varphi_{n},\left.\quad \varphi_{n}\right|_{\partial \Omega}=0 .
$$

This proves that $\varphi_{n}$ is an eigenfunction of the Schrödinger operator $-\nabla^{b} \cdot \nabla^{b}+$ $V_{n}^{E_{(n)}}+Q$ for the eigenvalue $E_{(n)}$. Moreover, since $\sigma_{(n)}=2$ in $B(1)$, it follows from (8) and (164) that

$$
\liminf _{n \rightarrow \infty}\left\|\varphi_{n}\right\|_{L^{2}(\Omega)} \geq \sqrt{2} \liminf _{n \rightarrow \infty}\left\|u_{n}\right\|_{L^{2}(B(1))} \geq \sqrt{2}\left\|u^{0}\right\|_{L^{2}(B(1))}=\frac{1}{2} .
$$

This inequality and eq. (166) together imply (155). Thus we have proven (i). 
Next, consider (ii). We start with the boundary-value problem for the acoustic equation

$$
\left(-g_{(n)}^{-1 / 2} \nabla^{b} \cdot \sigma_{(n)} \nabla^{b}+V_{n}^{E}+q^{E}+E\right) v_{n}^{E}(h)=0,\left.\quad v_{n}^{E}(h)\right|_{\partial \Omega}=h .
$$

Clearly

$$
v_{n}^{E}(h)=a_{n}^{E}(h) u_{(n)}+w_{n}^{E}(h), \quad \text { with }\left(w_{n}^{E}(h), u_{(n)}\right)_{L^{2}\left(\Omega, g_{(n)}^{1 / 2} d x\right)}=0,
$$

where, using the notations introduced in (129), (130), we have

$$
\begin{gathered}
a_{n}^{E}(h)=\left(h^{e}, u_{(n)}\right)_{L^{2}\left(g_{(n)}^{1 / 2} d x\right)}+\frac{1}{E-E_{(n)}}\left(F^{E}(h), u_{(n)}\right)_{L^{2}\left(g_{(n)}^{1 / 2} d x\right)}, \\
w_{n}^{E}(h)=h^{e}-\left(h^{e}, u_{(n)}\right)_{L^{2}\left(g_{(n)}^{1 / 2} d x\right)} u_{(n)}-\tilde{w}_{n}^{E}(h), \\
\tilde{w}_{n}^{E}(h)=\left(\mathcal{A}_{(n)}-E\right)^{-1}\left(F^{E}(h)-\left(F^{E}(h), u_{(n)}\right)_{L^{2}\left(g_{(n)}^{1 / 2} d x\right)} u_{(n)}\right) .
\end{gathered}
$$

Since $F^{E}(h)-\left(F^{E}(h), u_{(n)}\right) u_{(n)}$ and $u_{(n)}$ are orthogonal in $L^{2}\left(\Omega, g_{(n)}^{1 / 2} d x\right)$, and $\operatorname{dist}\left(E, \operatorname{spec}\left(\mathcal{A}_{(n)}\right) \backslash\left\{E_{(n)}\right\}\right)>d / 2$, it follows from (129) that, for $n$ large enough,

$$
\left\|\tilde{w}_{n}^{E}(h)\right\|_{L^{2}\left(\Omega, g_{(n)}^{1 / 2} d x\right)} \leq C,
$$

where $C$ is independent of $E \in\left(E^{0}-d / 4, E^{0}+d / 4\right)$ and $h$ satisfying $\|h\|_{H^{3 / 2}(\partial \Omega)} \leq$ 1. Note that in $\Omega \backslash B(2)$ the function $\tilde{w}_{n}^{E}(h)$ satisfies the equation

$$
\begin{gathered}
-\nabla^{b} \cdot \nabla^{b} \tilde{w}_{n}^{E}(h)=E \tilde{w}_{n}^{E}(h)+F^{E}(h)-\left(F^{E}(h), u_{(n)}\right)_{L^{2}\left(g_{m}^{1 / 2} d x\right)} u_{(n)}, \\
\left.\tilde{w}_{n}^{E}(h)\right|_{\partial \Omega}=0 .
\end{gathered}
$$

Thus, by boundary elliptic regularity, see Theorem 9.13 in [23],

$$
\left\|\tilde{w}_{n}^{E}(h)\right\|_{H^{2}(\Omega \backslash B(\rho))} \leq C_{\rho}, \quad 2<\rho<3 .
$$

This inequality, (129) and (130) together imply that $p_{(n)}^{E}(h)=\left(\partial_{v}+i v \cdot b\right) w_{n}^{E}(h)$ satisfies

$$
\left\|p_{(n)}^{E}(h)\right\|_{H^{1 / 2}(\partial \Omega)} \leq C_{0}, \text { for }\|h\|_{H^{3 / 2}(\partial \Omega)} \leq 1,
$$

if $n$ is large and $\left|E-E_{(n)}\right| \leq d / 2$. Finally, integration by parts shows that

$$
a_{n}^{E}(h)=\frac{1}{E-E_{(n)}} \int_{\partial \Omega} h \frac{\partial \overline{u_{(n)}}}{\partial v} d S=\frac{1}{E-E_{(n)}} \int_{\partial \Omega} h \frac{\partial \overline{\varphi(n)}}{\partial v} d S .
$$

The desired eq. (156) follows from the above equation together with eq. (167), (169) and (157), if we take into the account the relation (148) between $\psi_{(n)}^{E}(h)$ and $v_{n}^{E}(h)$. 
Remark 6.2. Theorem 6.1 means that, away from the cloaking structure, the eigenfunctions $\varphi_{(n)}$ converge to zero as $n \rightarrow \infty$, i.e. the $\varphi_{(n)}$ represent almost trapped modes, effectively vanishing near $\partial \Omega$. Physically speaking, we can say that if $E^{0}$ is an eigenvalue of the Schrödinger operator with Neumann boundary condition in the cloaked region $B(1)$, and we connect the interior and the exterior via the cloaking potential $V_{n}^{E}$ in the layer $B\left(R_{(n)}\right) \backslash B(1)$, with $R_{(n)} \rightarrow 1$ as $n \rightarrow \infty$, a particle under the influence of the combined potential on $B(3)$ is still largely confined (modulo standard tunneling) to the interior region, with a slight shift of the energy of the eigenmode from $E^{0}$ to $E_{(n)}$.

Moreover, for energies $E$ close to the values $E_{(n)}$ the presence of the cloaked region is very clearly seen in the boundary measurements of the DN map, so that the invisibility effect is compromised. On the other hand, at energies which are away from the $E_{(n)}$, the DN map for the potential $V_{(n)}^{E}+Q$ well approximates $\Lambda_{\text {out }}^{E}$, and thus the potential $Q$ is approximately cloaked.

\section{Numerical results}

Next we consider scattering problems for the Helmholtz and Schrödinger equations in the case when the magnetic potential vanishes, i.e. $b=0$.

The scattering problem for Helmholtz equation is

$$
\begin{gathered}
\left(\nabla \cdot \sigma_{(n)} \nabla+k^{2}(1+\alpha(x)) g_{(n)}^{1 / 2}\right) u^{\mathrm{tot}}=0 \text { in } \mathbb{R}^{3}, \\
u^{\mathrm{tot}}(x, k)=u^{\mathrm{in}}(x, k)+u^{\mathrm{sc}}(x, k)
\end{gathered}
$$

and for the Schrödinger equation

$$
\begin{gathered}
\left(-\nabla \cdot \nabla+V_{n}^{E}+Q-E\right) \psi^{\mathrm{tot}}=0 \text { in } \mathbb{R}^{3}, \\
\psi^{\mathrm{tot}}(x, E)=\psi^{\mathrm{in}}(x, E)+\psi^{\mathrm{sc}}(x, E),
\end{gathered}
$$

where $E>0, k=E^{1 / 2}$, the incident fields are $u^{\text {in }}(x, k)=\psi^{\text {in }}(x, E)=e^{i k \omega \cdot x}$, $|\omega|=1$, and the scattered fields satisfy the radiation condition

$$
\lim _{r \rightarrow \infty} r\left(\frac{\partial}{\partial r}-i k\right) u^{\mathrm{sc}}(x, k)=\lim _{r \rightarrow \infty} r\left(\frac{\partial}{\partial r}-i E^{1 / 2}\right) \psi^{\mathrm{sc}}(x, E)=0, \quad r=|x| .
$$

In following we consider $\alpha(x)$ that corresponds to a real bounded potential $Q$ supported in $B(1)$, that is, $\alpha(x)=-\left(E^{-1} Q(x)+3\right) / 4$. We assume that $Q(x)$ is such that $1+\alpha(x) \geq c_{0}>0$.

We consider also the solutions of the boundary value problems in $\Omega$ (note that we denote these solutions by $u$ and $\psi$, without using superscripts),

$$
\left(\nabla \cdot \sigma_{(n)} \nabla+k^{2}(1+\alpha(x)) g_{(n)}^{1 / 2}\right) u=0 \quad \text { in } \Omega,\left.\quad u\right|_{\partial \Omega}=h
$$


and for the Schrödinger equation

$$
\left(-\nabla \cdot \nabla+V_{n}^{E}+Q-E\right) \psi=0 \text { in } \Omega,\left.\quad u\right|_{\partial \Omega}=h .
$$

The solutions of these scattering and boundary value problems are related through a gauge transformation,

$$
\psi^{\mathrm{tot}}(x)=\sigma_{(n)}^{1 / 2}(x) u^{\mathrm{tot}}(x), \quad \psi(x)=\sigma_{(n)}^{1 / 2}(x) u(x)
$$

The computations are made without reference to physical units; for simplicity, we use $E=2$. The cloak corresponds to the parameter $R=1.005$ and inside the cloak we have located a spherically symmetric potential;

$$
Q(x)=Q_{\text {in }} \chi_{[0, R]}(|x|), \text { that is } \alpha(x)=-Q_{\text {in }}(4 E)^{-1} \chi_{[0, R]}(|x|)-3 / 4 \text {. }
$$

To illustrate the approximate cloaking, we used

$$
Q_{\text {in }}=1,
$$

and to obtain an almost trapped state,

$$
Q_{\text {in }}=-2.576
$$

In our numerical solution we have approximated $\sigma_{(n)}$ by a piecewise constant function consisting of 30 layers in the region $R<r<2$. The values of the conductivity in these layers are chosen as in the above sections. This corresponds to the case when the cloaking potential $V_{n}^{E}$ is a weighted sum of delta functions, and their derivatives, on spheres.

In the numerical solution of the problem, we represent the solution $u^{\text {tot }}$ and $u$ in terms of spherical harmonics $Y_{m}^{n}$ and Bessel functions up to order $N=7$ in each layer where the cloaking conductivity is constant. The transmission condition on the boundaries of these layers are solved numerically by solving linear equations. After this we compute the solution $\psi^{\text {tot }}$ and $\psi$ of the Schrödinger equation using the gauge transformation (174). 

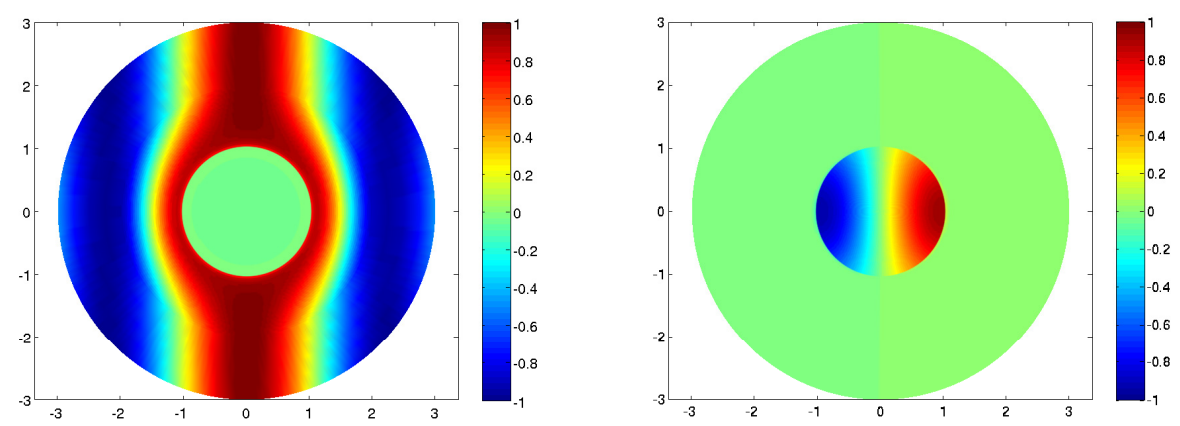

Figure 1. Solutions of acoustic equations. Left. Solution $u^{\text {tot }}$ of the scattering problem (170) restricted to ball $B(3)$, when a plane wave scatters from an approximate cloak in the case (175), i.e. when $k^{2}$ is away from the exceptional values $E_{(n)}$. Right. Almost trapped eigenfunction $u$ of the acoustic operator (172), with Dirichlet boundary condition, $h=0$, in the case (176), i.e. when $k^{2}$ is equal to the exceptional value $E_{(n)}$.
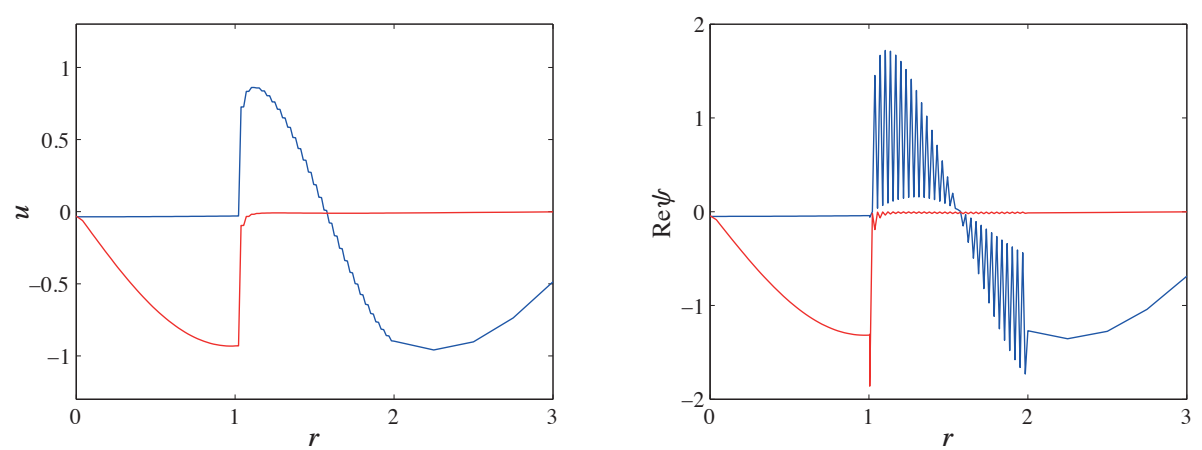

Figure 2. Radial profiles of solutions of acoustic and Schrödinger equations. Left. The solution $u^{\text {tot }}$ of the scattering problem $(170)$ on the line segment $L=\{(x, 0,0): x \in[0,3]\}$ in the case of Figure 1 (Left), where $k^{2}$ is far from the exceptional values $E_{(n)}$, is shown with the blue curve. Also, the eigenfunction $u$ of acoustic operator (173) on the line segment $L$ in the case of Figure 1 (Right), where $k^{2}$ is the exceptional value $E_{(n)}$, is shown with the red curve. Right. The solutions $\psi^{\text {tot }}$ and $\psi$ of the Schrödinger eq. (171) and (173) on the line segment $L$, obtained from the solutions on the left via gauge transformations (174).

\section{References}

[1] G. Allaire, Homogenization and two-scale convergence. SIAM J. Math. Anal. 23 (1992), no. 6, 1482-1518. MR 1185639 Zbl 0770.35005

[2] G. Allaire, A. Damlamian and U. Hornung, Two-scale convergence on periodic surfaces and applications. In A. Bourgeat, C. Carasso, S. Luckhaus and A. Mikelic (eds.), Mathematical Modelling of Flow through Porous Media, 15-25, Singapore, World Scientific, 1995. 
[3] A. Alú and N. Engheta, Cloaking a sensor. Phys. Rev. Lett. 102 (2009), 233901.

[4] H. Attouch, Variational convergence for functions and operators. Appl. Math. Series, Pitman, Boston, MA, 1984. MR 0773850 Zbl 0561.49012

[5] N. Aronszajn, A unique continuation theorem for solutions of elliptic partial differential equations or inequalities of second order. J. Math. Pures Appl. 36 (1957), 235-249 MR 0092067 Zbl 0084.30402

[6] A. Bensoussan, J.L. Lions and G. Papanicolaou, Asymptotic analysis for periodic structures. North-Holland, Amsterdam, 1978. MR 0503330 Zbl 0404.35001

[7] Y. Berezanskii, The uniqueness theorem in the inverse problem of spectral analysis for the Schrödinger equation. Trudy Moskov. Mat. Obsch. 7 (1958), 1-62, in Russian. MR 0101377

[8] A. Bukhgeim, Recovering a potential from Cauchy data. J. Inverse Ill-Posed Prob. 16 (2008), 19-33. MR 2387648 Zbl 1142.30018

[9] S. Chanillo, A problem in electrical prospection and an $n$-dimensional Borg-Levinson theorem. Proc. Amer. Math. Soc. 108 (1990), 761-767. MR 0998731 Zbl 0702.35035

[10] H.-Y. Chen and C. T. Chan, Transformation media that rotate electromagnetic fields. Appl. Phys. Lett. 90 (2007), 241105.

[11] H.-Y. Chen and C.T. Chan, Acoustic cloaking in three dimensions using acoustic metamaterials. Appl. Phys. Lett. 91 (2007), 183518.

[12] H.-Y. Chen and C.T. Chan, Electromagnetic wave manipulation using layered systems. Phys. Rev. B 78 (2008), 054204.

[13] H.-S. Chen, B.-I. Wu, B. Zhang and J.A. Kong, Electromagnetic wave interactions with a metamaterial cloak. Phys. Rev. Lett. 99 (2007), 063903.

[14] H. Chen, Y. Luo, L. Ran, J. A. Kong, and J. Zhang1, Design and analytically full-wave validation of the invisibility cloaks, concentrators, and field rotators created with a general class of transformations. Phys. Rev. B 77 (2008), 125127.

[15] A. Cherkaev, Variational methods for structural optimization, Appl. Math. Sci. 140, Springer-Verlag, 2000. MR 1763123

[16] S. Cummer and D. Schurig, One path to acoustic cloaking. New J. Phys. 9 (2007), 45.

[17] S. Cummer, J. Pendry, B.-I. Popa, M. Rahm1, D. Schurig, D. R. Smith, and A. Starr, Scattering theory derivation of a 3D acoustic cloaking shell. Phys. Rev. Lett. 100 (2008), 024301 .

[18] G. Dal Maso, An Introduction to $\Gamma$-convergence. Prog. in Nonlinear Diff. Eq. and their Appl. 8. Birkhäuser Boston, Inc., Boston, 1993. MR 1201152 Zbl 0816.49001

[19] L. Dolin, To the possibility of comparison of three-dimensional electromagnetic systems with nonuniform anisotropic filling. Izv. VUZov. Radiofizika 4 (1961), 964-967, in Russian

[20] L. Faddeev, Growing solutions of the Schrödinger equation. Dokl. Akad. Nauk SSSR 165 (1965), 514-517 (translation in Sov. Phys. Dokl. 10, 1033).

[21] M. Farhat, S. Guenneau, A.B. Movchan and S. Enoch, Achieving invisibility over a finite range of frequencies. Optics Express 16 (2008), 5656.

[22] D. Genov, C. Sun, S.Zhang, and X. Zhang, Cloaking of matter waves. Phys. Rev.Lett. 100 (2008), 123002. 
[23] D. Gilbarg and N. Trudinger, Elliptic Partial Differential Equations of Second Order. 2nd ed., Springer-Verlag, Berlin, 1983. MR 0737190 Zbl 0562.35001

[24] A. Greenleaf, Y. Kurylev, M. Lassas and G. Uhlmann, Full-wave invisibility of active devices at all frequencies. Comm. Math. Phys. 279 (2007), 749-789. MR 2336363 Zbl 1151.78006

[25] A. Greenleaf, Y. Kurylev, M. Lassas and G. Uhlmann, Electromagnetic wormholes and virtual magnetic monopoles from metamaterials. Phys. Rev. Lett. 99 (2007), 183901.

[26] A. Greenleaf, Y. Kurylev, M. Lassas and G. Uhlmann: Improvement of cylindrical cloaking with the SHS lining. Optics Expr. 15 (2007), 12717-12734.

[27] A. Greenleaf, Y. Kurylev, M. Lassas and G. Uhlmann, Electromagnetic wormholes via handlebody constructions. Comm. Math. Phys. 281 (2008), 369-385. MR 2410899 Zbl 1161.78003

[28] A. Greenleaf, Y. Kurylev, M. Lassas and G. Uhlmann, Comment on "Scattering theory derivation of a 3D acoustic cloaking shell”. Preprint 2008. arXiv:0801.3279.

[29] A. Greenleaf, Y. Kurylev, M. Lassas and G. Uhlmann, Isotropic transformation optics: approximate acoustic and quantum cloaking. New J. Phys. 10 (2008), 115024.

[30] A. Greenleaf, Y. Kurylev, M. Lassas and G. Uhlmann, Approximate quantum cloaking and almost trapped states. Phys. Rev. Lett. 101 (2008), 220404.

[31] A. Greenleaf, Y. Kurylev, M. Lassas, G. Uhlmann: Cloaking a Sensor via Transformation Optics. To appear in Physical Review E. Preprint 2009. arXiv:0912.1872v1.

[32] A. Greenleaf, Y. Kurylev, M. Lassas, G. Uhlmann: Invisibility and Inverse Problems. Bulletin of the American Mathematical Society 46 (2009), 55-97. MR 2457072 Zbl 1159.35074

[33] A. Greenleaf, M.Lassas, and G. Uhlmann, The Calderón problem for conormal potentials, I: Global uniqueness and reconstruction. Comm. Pure Appl. Math. 56 (2003), no. 3, 328352. MR 1941812 Zbl 1061.35165

[34] A. Greenleaf, M.Lassas, and G. Uhlmann, Anisotropic conductivities that cannot detected by EIT. Physiolog. Meas. (special issue on Impedance Tomography) 24 (2003), 413-420.

[35] A. Greenleaf, M. Lassas, and G. Uhlmann, On nonuniqueness for Calderón's inverse problem. Math. Res. Lett. 10 (2003), 685-693. MR 2024725

[36] P. Grinevich and R. Novikov, Transparent potentials at fixed energy in dimension two. Fixed-energy dispersion relations for the fast decaying potentials. Comm. Math. Phys. 174 (1995), 409-446. MR 1362172 Zbl 0843.35090

[37] A. Hendi, J. Henn and U. Leonhardt, Ambiguities in the scattering tomography for central potentials. Phys. Rev. Lett. 97 (2006), 073902.

[38] Y. Huang, Y. Feng and T. Jiang, Electromagnetic cloaking by layered structure of homogeneous isotropic materials. Optics Expr. 15 (2007), 11133-11141.

[39] T. Kato, Perturbation theory for linear operators. Springer-Verlag, 1980. Zbl 0435.47001

[40] H. Koch and D. Tataru, Carleman estimates and unique continuation for second-order elliptic equations with nonsmooth coefficients. Comm. Pure Appl. Math.54 (2001), 339360. MR 1809741 Zbl 1033.35025 
[41] R. Kohn, D. Onofrei, M. Vogelius and M. Weinstein, Cloaking via change of variables for the Helmholtz equation. Comm. Pure Appl. Math. 63 (2010),973-1016. MR 2642383 Zbl 1194.35505

[42] R. Kohn, H. Shen, M. Vogelius, and M. Weinstein, Cloaking via change of variables in electrical impedance tomography. Inver. Prob. 24 (2008), 015016. MR 2384775 Zbl 1153.35406

[43] A. Kufner and B. Opic, Hardy-type inequalities. Pitman Research Notes in Mathematics Series 219, Longman, 1990. MR 1069756 Zbl 0698.26007

[44] Y. Kurylev, M. Lassas and E. Somersalo, Maxwell's equations with a polarization independent wave velocity: direct and inverse problems. J. Math. Pures Appl. 86 (2006), 237-270. MR 2257731 Zbl 1134.35107

[45] M. Lassas, G. Uhlmann: Determining Riemannian manifold from boundary measurements. Annales Scientifiques de l'Ecole Normale Superieure. 34 (2001), 771-787. MR 1862026 Zbl 0992.35120

[46] R. Lavine and A. Nachman, The Faddeev-Lippmann-Schwinger equation in multidimensional quantum inverse scattering. In Inverse problems: an interdisciplinary study (Montpellier, 1986), 169-174, Adv. Electron. Electron Phys., Suppl. 19, Academic Press, London, 1987. MR 1005570

[47] H. Leinfelder and C. G. Simader, Schrödinger operators with singular magnetic vector potentials. Math Zeit. 176 (1981), 1-19. MR 0606167 Zbl 0468.35038

[48] U.Leonhardt, Optical conformal mapping. Science 312 (2006), 1777-1780. MR 2237569

[49] U. Leonhardt and T. Philbin, General relativity in electrical engineering. New J. Phys. 8 (2006), 247.

[50] R. Lipton, Homogenization and field concentrations in heterogeneous media. SIAM J. Math. Anal. 38 (2006), 1048-1059. MR 2274473 Zbl 1151.35007

[51] G. Milton, The Theory of Composites. Cambridge Univ. Press, 2001. MR 1899805 Zbl 0993.74002

[52] G. Milton, M. Briane, and J. Willis, On cloaking for elasticity and physical equations with a transformation invariant form. New J. Phys. 8 (2006), 248.

[53] A. Nachman, Reconstructions from boundary measurements. Ann. Math. 128 (1988), 531-576. MR 0970610 Zbl 0675.35084

[54] A. Nachman, Global uniqueness for a two-dimensional inverse boundary value problem. Ann. Math. 143 (1996), 71-96. MR 1370758

[55] A. Nachman, J. Sylvester and G. Uhlmann, An n-dimensional Borg-Levinson theorem. Comm. Math. Phys. 115 (1988), 595-605. MR 0933457 Zbl 0644.35095

[56] R. Newton, Construction of potentials from the phase shifts at fixed energy. J. Math. Phys. 3 (1962), 75-82. MR 0138364

[57] G. Nguetseng, A general convergence result for a functional related to the theory of homogenization. SIAM J.Math. Anal. 20 (1989), 608-623. MR 0990867 Zbl 0688.35007

[58] A. Norris, Acoustic cloaking theory. Proc. Royal Soc. A 464 (2008), 2411-2434. MR 2429553 Zbl 1186.74060 
[59] R. Novikov, Multidimensional inverse spectral problem for the equation $-\Delta \psi+(v(x)-$ $E u(x))=$ 0. Funkt. Anal. i Pril. 22 (1988), 11-22. MR 0976992

[60] R. Novikov, The inverse scattering problem at fixed energy for the three-dimensional Schrödinger equation with an exponentially decreasing potential. Comm. Math.Phys.161 (1994), 569-595. MR 1269391 Zbl 0803.35166

[61] P. Ola, L. Päivärinta and E. Somersalo, An inverse boundary value problem in electrodynamics. Duke Math. J. 70 (1993), 617-653. MR 1224101 Zbl 0804.35152

[62] J.B. Pendry, D. Schurig, and D.R. Smith, Controlling electromagnetic fields. Science 312 (2006), 1780-1782. MR 2237570

[63] S. A. Cummer, J. B. Pendry, M. Rahm, D. Schurig, and D. R. Smith, Optical Design of Reflectionless Complex Media by Finite Embedded Coordinate Transformations. Phys. Rev. Lett. 100 (2008), 063903.

[64] M. Reed and B. Simon. Methods of modern mathematical physics. I. Academic Press, New York, 1980. xv+400 pp. MR 0751959 Zbl 0459.46001

[65] T. Regge, Introduction to complex orbital method. Nuouvo Cimento 14 (1959), 951-976. MR 0143532 Zbl 0087.42903

[66] Z. Ruan, M. Yan, C. Neff and M. Qiu, Ideal cylindrical cloak: perfect but sensitive to tiny perturbations. Phys. Rev. Lett. 99 (2007), 113903.

[67] P. Sabatier, Asymptotic properties of the potentials in the inverse-scattering problem at fixed energy. J. Math. Phys. 7 (1966), 1515-1531. MR 0207328

[68] B. Simon, Maximal and minimal Schrödinger forms. J. Operator Theory 1 (1979), 37-47. MR 0526289 Zbl 0446.35035

[69] Z. Sun and G. Uhlmann, Generic uniqueness for an inverse boundary value problem. Duke Math. J. 62 (1991), 131-155. MR 1104326 Zbl 0728.35132

[70] J. Sylvester and G. Uhlmann, A global uniqueness theorem for an inverse boundary value problem. Ann. Math. 125 (1987), 153-169. MR 0873380 Zbl 0625.35078

[71] G. Uhlmann, Inverse boundary value problems and applications. Astérisque 207 (1992) 153-211. MR 1205179 Zbl 0787.35123

[72] A. Ward and J. Pendry, Refraction and geometry in Maxwell's equations. J. Modern Opt. 43 (1996), 773-793. MR 1390260 Zbl 0941.78514

[73] R. Weder, A rigorous analysis of high-order electromagnetic invisibility cloaks. J. Phys. A: Math. and Theor. 41 (2008), 065207. MR 2435934 Zbl 1132.35488

Received January 28, 2010, revised October 21, 2010

Allan Greenleaf, Department of Mathematics, University of Rochester, Rochester, NY 14627, U.S.A.

E-mail: allan@math.rochester.edu

Yaroslav Kurylev, Department of Mathematical Sciences, University College, London,

WC1E 6BT, U.K.

E-mail: y.kurylev@ucl.ac.uk 
Matti Lassas, University of Helsinki, Department of Mathematics and Statistics, P.O. Box 68, 00014 University of Helsinki, Finland

E-mail: matti.lassas@helsinki.fi

Gunther Uhlmann, Department of Mathematics, University of California, Irvine, Irvine, CA 92697, U.S.A.

E-mail: guhlmann@math.uci.edu

Department of Mathematics, University of Washington, Seattle, WA 98195, U.S.A.

E-mail: gunther@math.washington.edu 